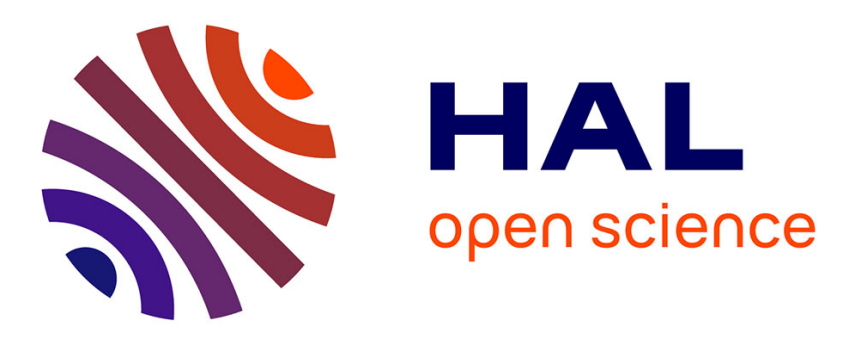

\title{
Synthesis of Norbornene-Based Phosphine-Stabilized Silylium Ions Behaving as Masked Frustrated Lewis Pairs
}

\author{
Aymeric Dajnak, Eddy Maerten, Nathalie Saffon-Merceron, Antoine \\ Baceiredo, Tsuyoshi Kato
}

\section{To cite this version:}

Aymeric Dajnak, Eddy Maerten, Nathalie Saffon-Merceron, Antoine Baceiredo, Tsuyoshi Kato. Synthesis of Norbornene-Based Phosphine-Stabilized Silylium Ions Behaving as Masked Frustrated Lewis Pairs. Organometallics, 2020, 39 (18), pp.3403-3412. 10.1021/acs.organomet.0c00489 . hal-03408869

\section{HAL Id: hal-03408869 https://hal.science/hal-03408869}

Submitted on 29 Oct 2021

HAL is a multi-disciplinary open access archive for the deposit and dissemination of scientific research documents, whether they are published or not. The documents may come from teaching and research institutions in France or abroad, or from public or private research centers.
L'archive ouverte pluridisciplinaire HAL, est destinée au dépôt et à la diffusion de documents scientifiques de niveau recherche, publiés ou non, émanant des établissements d'enseignement et de recherche français ou étrangers, des laboratoires publics ou privés. 


\title{
Synthesis of Norbornene Based-Phosphine-Stabilized Silylium Ions Behaving as Masked Frustrated Lewis Pairs.
}

\author{
Aymeric Dajnak, ${ }^{a}$ Eddy Maerten, ${ }^{* a}$ Nathalie Saffon-Merceron, ${ }^{\mathrm{b}}$ Antoine Baceiredo, ${ }^{\mathrm{a}}$ Tsuyoshi Kato*a \\ a Université de Toulouse, UPS, and CNRS, LHFA UMR 5069, 118 route de Narbonne, 31062 Toulouse (France) \\ b Université de Toulouse, UPS, and CNRS, ICT FR2599 118 route de Narbonne, 31062 Toulouse (France)
}

\begin{abstract}
A novel architecture of phosphine-stabilized silylium ions is described. The peculiar moderate flexible framework allows an efficient donation of the phosphine group toward the electron deficient silicon center thus providing a very good stabilization of the corresponding silylium ions. The P-Si interaction was studied in solution and in the solid state by NMR spectroscopy and X-ray diffraction analysis, respectively. The reactivity of silylium ions is easily improved using less donating phosphine ligands. Of particular interest, thanks to the flexible ligand framework, phosphine-stabilized silylium ion 5f behaves as a frustrated Lewis pair instead of a simple Lewis acid and exhibits an original ambiphilic reactivity with carbonyl derivatives that allows the synthesis of seven- to nine-membered ring heterocycles.
\end{abstract}

With the synthesis of the first free silylium ion I, the groups of Reed and Lambert have achieved a major synthetic challenge. ${ }^{1}$ Indeed, because of their exceptionally high Lewis acidity, silylium ions have remained for a long time elusive species. ${ }^{2}$ To take advantage of these unique species in synthesis or catalysis, their reactivity needs to be tamed. ${ }^{3}$ The study of these species has been facilitated by the use of a Lewis Base that can intramolecularly interact with the silylium center, to afford a good balance between reactivity and stability (II, Figure 1). ${ }^{4}$ Following this strategy, several different types of donor groups have been introduced to achieve this intramolecular stabilization (III-VI, Figure 1).
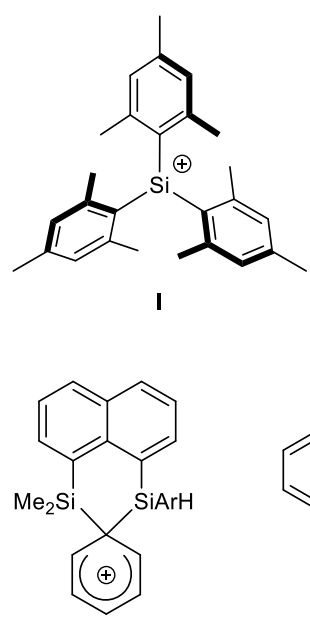

IV

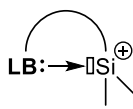

II

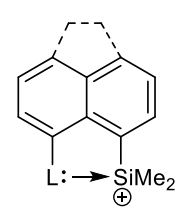

V

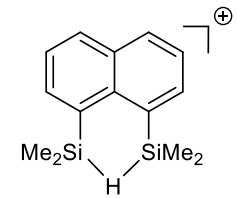

III

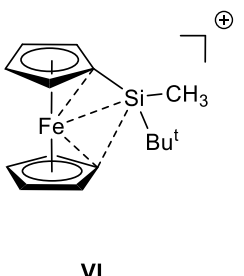

VI
The applications of these species have generated great deal of interest in recent years, especially in the field of catalysis. For instance, hydrodefluorination (HDF) reactions of alkylfluorides were found to be efficiently catalyzed by intramolecularly stabilized silyl cations III, IV and V. ${ }^{5}$ These species are also capable of catalyzing Diels-Alder reactions, and the ferrocene-stabilized system VI developed by Oestreich group is particularly efficient for the low-temperature cycloaddition reactions with $\alpha, \beta$-unsaturated ketones. ${ }^{6}$ Interestingly, triarylsilylium ions such as $\mathbf{I}$ have been used for the rational design of new frustrated Lewis pair (FLP) systems able to activate dihydrogen and to sequester carbon dioxide. ${ }^{7}$

One of our main objectives is the stabilization of electron deficient species, ${ }^{8}$ and recently, a framework based on norbornene was designed to stabilize silylenes using phosphines as Lewis base. ${ }^{9}$ Of special interest, we have shown that, due to the weak coordination of phosphine ligand, phosphine-stabilized silylenes remain highly reactive. Moreover, they present, to some extent, "transition metal-like behavior" as demonstrated by the reversible binding of ethylene to the $\mathrm{Si}$ (II) center ${ }^{10}$ and the reversible oxidation addition/reductive elimination of $\mathrm{R}_{3} \mathrm{SiH}$ at the $\mathrm{Si}(\mathrm{II})$ center. ${ }^{11}$ The key features of this system are the moderate structural flexibility as well as the rigid planar structure of the enamino phosphine fragment, which results in a particular $\mathrm{P} \rightarrow \mathrm{Si}$ interaction. We have thus considered the synthesis of the novel phosphine-stabilized silylium ions $\mathbf{4}$, which despite of a strong P-Si interaction behave as frustrated Lewis pairs and allows the synthesis of seven- to nine-membered heterocycles.

Figure 1. First free silylium ion and intramolecularly stabilized silylium ions. 
A straightforward synthesis of the silylium precursors, the silylated amino(phosphino) derivatives $\mathbf{2}$, has been developed from the corresponding iminophosphines 1 (Scheme 1). ${ }^{9}$

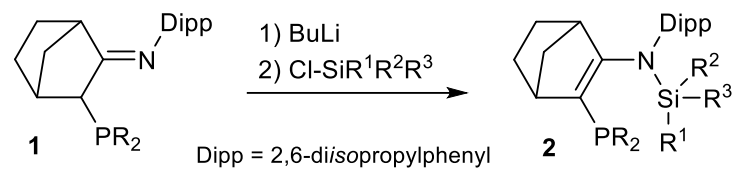

2a: $R^{1}=H, R^{2}=R^{3}=M e$
$\mathbf{2 b}: R^{1}=C l, R^{2}=R^{3}=M e$
$\mathbf{2 c}: R^{1}=R^{2}=C l, R^{3}=M e$
2d: $R^{1}=R^{2}=C l, R^{3}=H$
2e: $R^{1}=R^{2}=R^{3}=C l$
2f: $R^{1}=C l, R^{2}=R^{3}=M e$
2g: $R^{1}=R^{2}=C l, R^{3}=M e$

Scheme 1. Synthesis of silylium precursors 2.

Attempts to generate silylium ions via the classical BartlettSchneider-Condon method ${ }^{2}$ using trityl tetrakis(pentafluorophenyl)borate have failed. Rather than the hydride abstraction, the phosphonium salt $\mathbf{3}$ was obtained nearly quantitatively, by the reaction of the trityl cation at the 4-position of a phenyl group (Scheme 2). The same type of reaction between bulky phosphines $\left(\mathrm{P} \mathrm{Bu}_{3}\right.$ or $\left.\mathrm{PCy}_{3}\right)$ and trityl salts was already observed by Stephan et al.. ${ }^{12}$

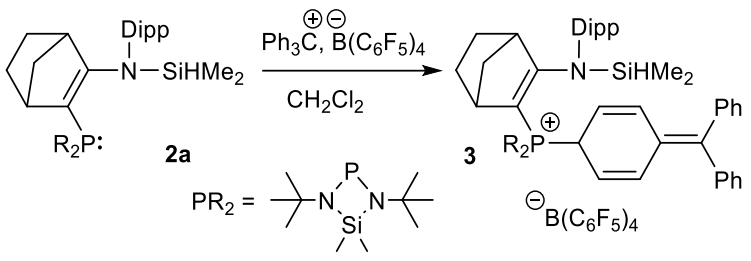

Scheme 2. Reaction of $\mathbf{2 a}$ with trityl cation.

Phosphonium salt 3 was fully characterized by NMR spectroscopy and its structure was confirmed by X-Ray diffraction analysis (Figure 2). The P1-C32 bond length is classical for a single bond, and the cyclohexadiene ring shows alternate single and double bond lengths, planar environments around C35 and C38 (respectively $\Sigma^{\circ}=359.9$ and $360.0^{\circ}$ ) and a tetrahedral environment at $\mathrm{C} 32$.

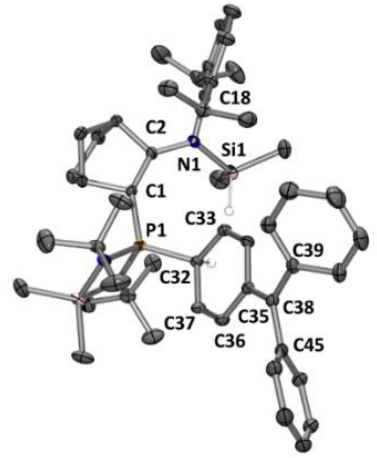

Figure 2. Molecular structure of 3 . Thermal ellipsoids represent $30 \%$ probability. $\mathrm{H}$ atoms (except on $\mathrm{Si} 1$ and $\mathrm{C} 32$ ), solvent molecules and counterion $\left[B\left(\mathrm{C}_{6} \mathrm{~F}_{5}\right)_{4}\right]^{-}$were omitted for clarity. Selected bond lengths $[\AA] \mathrm{A}: \mathrm{P} 1-\mathrm{C} 1$ 1.777(2), C1-C2 1.380(3), C2-N1 1.391(3), N1-C18 1.463(3), P1-C32 1.841(2), C32 C33 1.493(3), C33-C34 1.337(4), C34-C35 1.463(3), C35-C36 1.460(4), C36C37 1.339(4), C37-C32 1.508(3), C35-C38 1.372(3), C38-C39 1.486(4), C38C45 1.484(3).

The expected silylium ions $\mathbf{4}$ can be easily obtained following the Kira's and Sakurai's methodology, ${ }^{13}$ starting from the corresponding chlorosilanes $\mathbf{2 b - e}$, just by halide abstraction using potassium borate salt (Scheme 3 ).

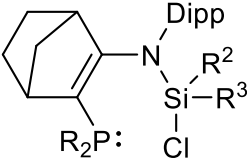

$2 b-g$

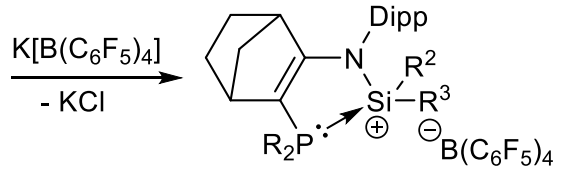

4b-e $\left(R_{2} P=>N_{S i}^{P} N \in\right)$

or $5 \mathbf{f}, \mathbf{g}\left(\mathrm{R}_{2} \mathrm{P}=\mathrm{Ph}_{2} \mathrm{P}\right)$
Scheme 3. Preparation of silylium ions $4 b-e$ and $5 f, g$

In order to study the phosphorus-silicon interaction, several silylium ions were prepared (4b-e), and characterized in solution and in the solid state. The main spectroscopic data are summarized in Table 1.

Table 1. NMR data of silylium ions $4 \mathbf{b}-\mathbf{e}$ (in $\mathrm{CD}_{2} \mathrm{Cl}_{2}$ at $298 \mathrm{~K}$ ).

\begin{tabular}{cccccc}
\hline Cpd & $\mathrm{R}^{2}$ & $\mathrm{R}^{3}$ & ${ }^{31} \mathrm{P}$ & ${ }^{29} \mathrm{Si}$ & $J_{\mathrm{PSi}}[\mathrm{Hz}]$ \\
\hline $\mathbf{4 b}$ & $\mathrm{Me}$ & $\mathrm{Me}$ & 39.6 & 6.1 & 132.0 \\
$\mathbf{4 c}^{[\mathrm{a}]}$ & $\mathrm{Cl}$ & $\mathrm{Me}$ & 30.2 and 30.0 & -0.3 and -2.1 & 172.6 and 171.2 \\
$\mathbf{4 d}^{[\mathrm{b}]}$ & $\mathrm{Cl}$ & $\mathrm{H}$ & 28.4 & -22.9 & 169.5 \\
$\mathbf{4 e}$ & $\mathrm{Cl}$ & $\mathrm{Cl}$ & 21.8 & -21.6 & 237.4 \\
\hline
\end{tabular}

Cpd = compound; [a] d.r. = 50:50. [b] only one diastereomer is formed. Chemical shift in ppm and coupling constants in $\mathrm{Hz}$.

Silylium ions $\mathbf{4} \mathbf{c}$ and $\mathbf{4 d}$ with two different substituents on the Si atom $\left(\mathbf{4 c}: \mathrm{R}^{2}=\mathrm{Cl}, \mathrm{R}^{3}=\mathrm{Me}, \mathbf{4 d}: \mathrm{R}^{2}=\mathrm{Cl}, \mathrm{R}^{3}=\mathrm{H}\right)$ were 
expected to be formed as diastereomer couples since the silylium center is stereogenic. A 50:50 diastereomeric ratio was obtained for $\mathbf{4} \mathbf{c}$ but in the case of $\mathbf{4 d}$, a single diastereomer is formed. Regarding the strong phosphine-silylium ion interaction (vide infra), the epimerization of $\mathbf{4}$ at the chiral silicon center through the P-Si bond dissociation is difficult to be considered. Instead, the diastereoselective formation of $\mathbf{4 d}$ is probably due to a selective $\mathrm{Cl}$-abstraction from $\mathbf{2} \mathbf{d}$ featuring a pentacoordinate $\mathrm{Si}$ center bearing two different $\mathrm{Cl}$ atoms (apical and equatorial positions). Indeed, the pentacoordinate nature of the $\mathrm{Si}$ atom in $\mathbf{2 d}$ with a strong $\mathrm{P} \rightarrow \mathrm{Si}$ interaction, was clearly indicated by the significantly highfield-shifted signals in ${ }^{29} \mathrm{Si}-$ and ${ }^{31} \mathrm{P}-\mathrm{NMR}\left({ }^{29} \mathrm{Si}:-87.2 \mathrm{ppm},{ }^{31} \mathrm{P}: 43.1 \mathrm{ppm}\right)$ with a large $J_{\text {Si-P }}$ coupling constant $(223.8 \mathrm{~Hz})$ compared to those observed for $\mathbf{2 c}$ showing typical silane and phosphine signals $\left({ }^{29} \mathrm{Si}:-5.5 \mathrm{ppm}\right.$, ${ }^{31} \mathrm{P}$ : $\left.111.3 \mathrm{ppm}\right)$ in agreement with a tetracoordinate $\mathrm{Si}$ atom without $\mathrm{P} \rightarrow \mathrm{Si}$ interaction (Scheme 4). Note that, 2d exists as a single diastereomer presenting a pentacoordinate $\mathrm{Si}$ center with a strongly electron-withdrawing $\mathrm{Cl}$ atom at the apical position and another $\mathrm{Cl}$ atom at the less-hindered equatorial position. The formation of $\mathbf{4 d}$ should exclusively takes place, keeping the configuration at the Si center, via the cleavage of more strongly polarized apical $\mathrm{Si}-\mathrm{Cl}$ bond in $\mathbf{2 d}$, which explains the high diastereoselectivity of the reaction. In contrast, in the case of $\mathbf{2 c}$, due to the free-rotation at the N-Si bond axis because of the absence of $\mathrm{P} \rightarrow \mathrm{Si}$ interaction, the abstraction of $\mathrm{Cl}$ anion is not selective and, thus, affords $\mathbf{4 c}$ as a mixture of two diastereomers.

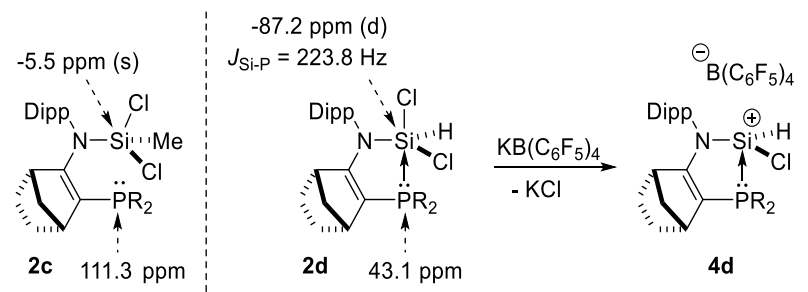

Scheme 4. ${ }^{29} \mathrm{Si}-$ and ${ }^{31} \mathrm{P}-\mathrm{NMR}$ chemical shifts of $\mathbf{2 c}$ and $2 \mathrm{~d}$ and the diastereoselective formation of $\mathbf{4 d}$.

The analysis of these spectroscopic data indicates that silylium 4e, which bears two strong electron-withdrawing chloride atoms, shows the strongest $\mathrm{P}-\mathrm{Si}$ interaction as indicated by a relatively shielded ${ }^{31} \mathrm{P}$ NMR chemical shift $(\delta=21.8 \mathrm{ppm})$ and a large silicon-phosphorus coupling constant $\left(J_{\mathrm{PSi}}=237.4\right.$ $\mathrm{Hz}$ ). The ${ }^{29} \mathrm{Si}$ NMR chemical shift is also a good probe to evaluate the Lewis acidity of silylium ions. ${ }^{14}$ In this series, $\mathbf{4 b}$ exhibits an enhanced Lewis acidity as indicated by the ${ }^{29} \mathrm{Si}$ NMR chemical shift which appears of at $\delta=6.1 \mathrm{ppm}$, among the more deshielded signals and the smallest silicon-phosphorus coupling constant $\left(J_{\mathrm{PSi}}=132.0 \mathrm{~Hz}\right)$. The four silylium ions $4 \mathrm{~b}$-e synthesized were also characterized, in the solid state, by X-ray diffraction analysis (Figure 3 and Table 2).

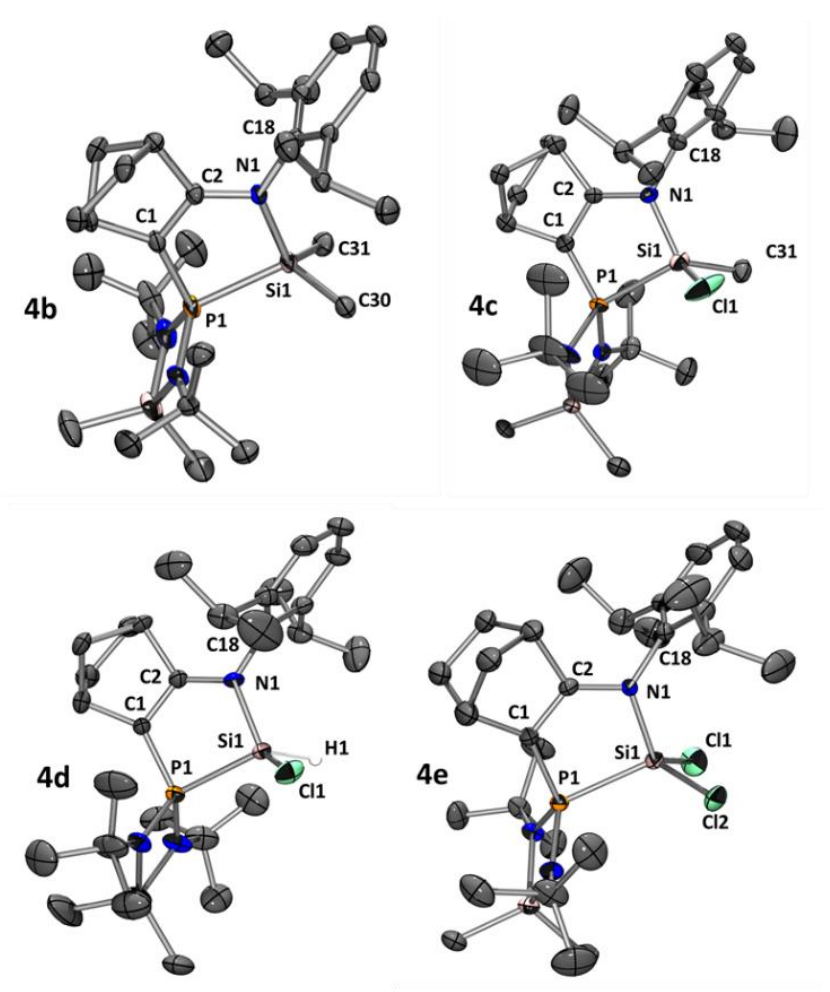

Figure 3. Molecular structure of $\mathbf{4 b}, \mathbf{4 c}, \mathbf{4 d}$ and $\mathbf{4 e}$. Thermal ellipsoids represent $30 \%$ probability. Counter ions, solvent molecules, disordered atoms and hydrogen atoms were omitted for clarity (except the hydrogen atom on the silicon atom of $\mathbf{4 d}$ ).

Table 2. Main bond lengths (in $[\AA]$ ) obtained from X-Ray diffraction analysis of 4b-4e.

\begin{tabular}{|c|c|c|c|c|c|c|c|}
\hline Cpd & P1C1 & $\mathrm{C} 1 \mathrm{C} 2$ & C2N1 & N1Si1 & Si1P1 & $\mathrm{Si}_{1} \mathrm{R}^{2}$ & $\mathrm{Si}_{1} \mathrm{R}^{3}$ \\
\hline $4 b$ & $\begin{array}{c}1.730 \\
(3)\end{array}$ & $\begin{array}{c}1.369 \\
(4)\end{array}$ & $\begin{array}{c}1.371 \\
(4)\end{array}$ & $\begin{array}{c}1.781 \\
(3)\end{array}$ & $\begin{array}{c}2.322 \\
(1)\end{array}$ & $\begin{array}{c}1.844 \\
(4) \\
\mathrm{Si}-\mathrm{CH}_{3}\end{array}$ & $\begin{array}{c}1.844 \\
(4) \\
\mathrm{Si}-\mathrm{CH}_{3}\end{array}$ \\
\hline $4 c$ & $\begin{array}{c}1.708 \\
(12)\end{array}$ & $\begin{array}{c}1.383 \\
(16)\end{array}$ & $\begin{array}{c}1.423 \\
(16)\end{array}$ & $\begin{array}{c}1.731 \\
(11)\end{array}$ & $\begin{array}{c}2.302 \\
(7)\end{array}$ & $\begin{array}{l}1.952 \\
(13) \\
\mathrm{Si}-\mathrm{Cl}\end{array}$ & $\begin{array}{c}1.73 \\
(3) \\
\mathrm{Si}-\mathrm{CH}_{3}\end{array}$ \\
\hline $4 d$ & $\begin{array}{c}1.737 \\
(9)\end{array}$ & $\begin{array}{c}1.370 \\
(10)\end{array}$ & $\begin{array}{c}1.355 \\
(6)\end{array}$ & $\begin{array}{c}1.734 \\
(4)\end{array}$ & $\begin{array}{c}2.287 \\
(2)\end{array}$ & $\begin{array}{c}2.067 \\
(2) \\
\mathrm{Si}-\mathrm{Cl}\end{array}$ & $\begin{array}{c}1.44 \\
(8) \\
\mathrm{Si}-\mathrm{H}\end{array}$ \\
\hline $4 e$ & $\begin{array}{c}1.711 \\
(4)\end{array}$ & $\begin{array}{c}1.368 \\
(5)\end{array}$ & $\begin{array}{c}1.368 \\
(4)\end{array}$ & $\begin{array}{c}1.730 \\
(3)\end{array}$ & $\begin{array}{c}2.293 \\
(1)\end{array}$ & $\begin{array}{c}2.014 \\
(1) \\
\mathrm{Si}-\mathrm{Cl}\end{array}$ & $\begin{array}{c}2.011 \\
(1) \\
\mathrm{Si}-\mathrm{Cl}\end{array}$ \\
\hline
\end{tabular}

The X-Ray diffraction data are in good agreement with the spectroscopic data. Indeed, the longest Si1P1 bond length $[2.322(1) \AA]$ of the series was found in $\mathbf{4 b}$, suggesting a weak $\mathrm{Si}-\mathrm{P}$ interaction, in other words, the stronger Lewis acid of the series. Noteworthy, the modification of the silylium center substituent $\left(\mathrm{R}^{2}\right.$ and $\left.\mathrm{R}^{3}\right)$ has a rather weak impact on the Si-P interaction.

In order to reduce the $\mathrm{Si}-\mathrm{P}$ interaction, we have considered the use of a less electron donating phosphine fragment. Therefore, the cyclic diaminophosphino group was replaced by a diphenylphosphino moiety, and the corresponding silylium ions 
5f,g $\left(\mathrm{PR}_{2}=\mathrm{PPh}_{2}\right)$ have been prepared using the same synthetic methodology as for 4 (Scheme 3).

The new silylium ions 5 were obtained in nearly quantitative yields, and in the case of $\mathbf{5 g}\left(\mathrm{R}^{2}=\mathrm{Cl}, \mathrm{R}^{3}=\mathrm{Me}\right)$ as a mixture of two diastereomers in a 76:24 ratio. The spectroscopic data are summarized in Table 3. As expected, the weaker donation of the diphenylphosphino group is evidenced by deshielded ${ }^{29} \mathrm{Si}$ NMR signals at $\delta=27.2 \mathrm{ppm}$ for $\mathbf{5 f}$ and $\delta=15.4$ and $15.3 \mathrm{ppm}$ for $\mathbf{5 g}$ compared to those observed for the corresponding silylium ions $\mathbf{4 b , c}$ stabilized by a more strongly electron donating diaminophosphino ligand (4b: $\delta=6.1 \mathrm{ppm}, \mathbf{4 c}: \delta=-0.3$ and $2.1 \mathrm{ppm})$. Again, the smallest $J_{\mathrm{PSi}}$ coupling constant is observed for silylium $\mathbf{5 f}\left(\mathrm{R}^{2}=\mathrm{R}^{3}=\mathrm{Me}\right)$.

Table 3. Experimental NMR parameters of silylium ions $\mathbf{5 f - g}$ (in $\mathrm{CD}_{2} \mathrm{Cl}_{2}$ at 298 $\mathrm{K})$.

\begin{tabular}{cccccc}
\hline Cpd & $\mathrm{R}^{2}$ & $\mathrm{R}^{3}$ & ${ }^{31} \mathrm{P}$ & ${ }^{29} \mathrm{Si}$ & $\mathrm{JPSi}[\mathrm{Hz}]$ \\
\hline $\mathbf{5 f}$ & $\mathrm{Me}$ & $\mathrm{Me}$ & -20.8 & 27.2 & 82.1 \\
$\mathbf{5 \mathbf { g } ^ { [ a ] }}$ & $\mathrm{Cl}$ & $\mathrm{Me}$ & -24.3 and -24.1 & 15.4 and 15.3 & 124.6 and 117.4 \\
\hline
\end{tabular}

Cpd = compound; [a] d.r. = 76:24. Chemical shift in ppm and coupling constants in $\mathrm{Hz}$.

Although, the ability of these two families of silylium ions as catalyst of Diels Alder reaction between cyclohexadiene or 2,3dimethylbutadiene and methyl acrylate $(10 \mathrm{~mol} \%$ of silylium 4 or 5 in $\left.\mathrm{CDCl}_{3}\right)^{4 a, 6,15}$ was tested, no trace of the expected cycloadducts could be detected by NMR spectroscopy, after several hours at $80{ }^{\circ} \mathrm{C}$. Interestingly, in the case of $\mathbf{5 f}$, the ${ }^{31} \mathrm{P}$ NMR analysis shows the complete disappearance of the silylium signal $(-20.8 \mathrm{ppm})$ and a new single signal appeared at $\delta=20.9$ ppm. In fact, silylium ion $\mathbf{5 f}$ reacts with one equivalent of methyl acrylate at $50{ }^{\circ} \mathrm{C}$, leading to an insertion reaction of the conjugated carbonyl into the $\mathrm{P} \rightarrow \mathrm{Si}$ dative bond. Silylium cation 5f, acting as a frustrated Lewis pairs (FLP), ${ }^{7 b, 16}$ affords quantitatively an original 9-membered heterocycle 6, which loses the Lewis acid character (silane structure), probably explaining the absence of any catalytic activity (Scheme 5).

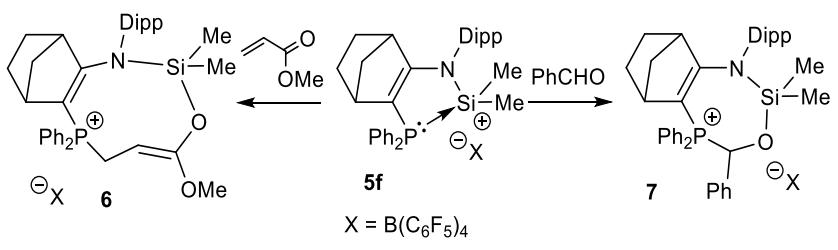

Scheme 5. Reaction of silylium ion $\mathbf{5 f}$ with carbonyl derivatives.

The ${ }^{13} \mathrm{C}$ NMR is particularly informative with the peculiar chemical shift of the ketene acetal type part $\mathrm{CH}=\mathrm{C}$ of $\mathbf{6}$, chemical shift at $\delta=67.8 \mathrm{ppm}\left(J_{\mathrm{CP}}=10.6 \mathrm{~Hz}\right)$ for primary- and at $\delta$ $=156.9 \mathrm{ppm}\left(J_{\mathrm{CP}}=5.5 \mathrm{~Hz}\right)$ for the quaternary-carbon atoms respectively. In the same vein, we observed at room temperature, the immediate and quantitative insertion of benzaldehyde into the Si-P bond of silylium 5f. ${ }^{17}$ The corresponding 7-membered ring 7 was isolated as a mixture of two diastereomers in a 68:32 ratio, and fully characterized by NMR spectroscopy and the structure was confirmed by X-ray diffraction analysis (Figure 4). The high reactivity of $\mathbf{5 f}$ as a masked FLP can be explained by the weak $\mathrm{P} \rightarrow \mathrm{Si}$ interaction as well as the moderate flexibility of ligand framework. Indeed, such a flexibility of ligand was clearly confirmed by the significantly elongated P1-Si1 distance (3.624 $\AA$ in 7 vs $2.322 \AA$ in $\mathbf{4 b}$ ) and the associated widen interior angles of P1-C1-C2-N1-Si1 fragment (P1-C1-C2 $132.36^{\circ}, \mathrm{C} 1-\mathrm{C} 2-\mathrm{N} 1130.78^{\circ}$ and $\left.\mathrm{C} 2-\mathrm{N} 1-\mathrm{Si} 1122.63^{\circ}\right)$ in 7 , compared with those of the phosphine-silylium ion complex $4 \mathbf{b}(\mathrm{P} 1-$ C1-C2 $116.82^{\circ}, \mathrm{C} 1-\mathrm{C} 2-\mathrm{N} 1125.50^{\circ}$ and $\left.\mathrm{C} 2-\mathrm{N} 1-\mathrm{Si} 1113.84^{\circ}\right)$. However, $\mathbf{5 f}$ is not enough reactive to activate $\mathrm{H}_{2}$ as observed for the previously reported frustrated Lewis pairs constituted of free-silylium ion and a bulky phosphine. It should be noted that, in contrast to our system, the phosphine-stabilized silylium ion with a naphthalene-based rigid ligand framework (Type $\mathbf{V}$ in Figure 1) is very stable and no reactivity of such species has been described to date. ${ }^{18}$ Similar FLP type reactions were also observed for phosphine-stabilized germylene and stannylenes with a strained three-membered cyclic structure. ${ }^{19}$

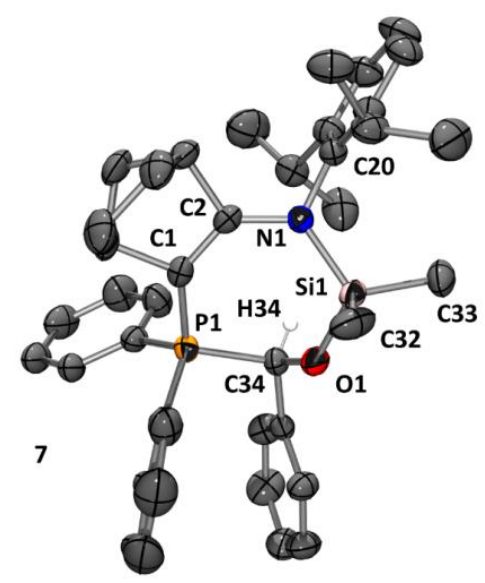

Figure 4. Molecular structure of 7 . Thermal ellipsoids represent $30 \%$ probability. Counter ion, disordered atoms and hydrogen atoms were omitted for clarity (except H34). Selected bond lengths [Å] and angles [ $\left.{ }^{\circ}\right]$ : P1-C1 1.746(2), C1-C2 1.363(3), C2-N1 1.386(2), N1-Si1 1.763(2), Si1-C32 1.841(3), Si1-C33 1.844(2), Si1-O1 1.650(2), C34-O1 1.393(2), P1-C34 1.864(2), C34-C35 1.509(3); P1-C1-C2 132.36(15), C1-C2-N1 130.78(17), C2-N1Si1 122.63(13), N1-Si1-O1 108.17(7), Si1-O1-C34 129.51(13), O1-C34P1 103.54(13), C34-P1-C1 109.32(9).

The reactions leading to the formation of cyclic phosphonium borates $\mathbf{6}$ and $\mathbf{7}$ are not reversible. Indeed, no evolution occurs after prolonged heating of the reaction mixtures. These adducts are so stable that silylium $\mathbf{5 f}$ could not be regenerated when additional reactants such as phenylsilane were added.

In conclusion, a new type of phosphine-stabilized-silylium ion, based on a norbornene scaffold, was described. As expected, the moderate structural flexibility favours the enamino phosphine donation to electrophilic silicon center, affording stable silylium ions. Phosphine-silicon interactions were studied both in solution and in the solid state. The reactivity of silylium ions is easily improved using a weaker donating phosphine ligand. Of particular interest, thanks to the flexible ligand framework, and despite of an efficient intramolecular stabilization, the base-stabilized silylium ions $\mathbf{5 f}$ behaves as frustrated Lewis pairs and allows the synthesis of seven- to nine-membered heterocycles. Further structural modifications are actually being 
performed to improve the potential of this new class of silylium ions.

\section{EXPERIMENTAL SECTION}

General Procedures. All manipulations were performed under an inert atmosphere of argon by using standard Schlenk techniques or high-pressure NMR tube techniques. Dry and oxygen-free solvents were used. ${ }^{1} \mathrm{H},{ }^{11} \mathrm{~B},{ }^{13} \mathrm{C},{ }^{19} \mathrm{~F},{ }^{28} \mathrm{Si}$ and ${ }^{31} \mathrm{P}$ NMR spectra were recorded on Brucker Avance II $300 \mathrm{MHz}$, Avance III HD $400 \mathrm{MHz}$ and Avance I and II $500 \mathrm{MHz}$ spectrometers. ${ }^{1} \mathrm{H},{ }^{29} \mathrm{Si}$ and ${ }^{13} \mathrm{C}$ NMR chemical shifts are reported in parts per million (ppm) relative to $\mathrm{Me}_{4} \mathrm{Si}$ as internal standard. ${ }^{11} \mathrm{~B}$ NMR downfield chemical are expressed in ppm relative to $\mathrm{BF}_{3} \cdot \mathrm{Et}_{2} \mathrm{O}$. ${ }^{19} \mathrm{~F}$-chemical shifts were reported in ppm relative to $\mathrm{CFCl}_{3}$ as an external standard. ${ }^{31} \mathrm{P}$ NMR chemical shifts are expressed in $\mathrm{ppm}$ relative to $85 \% \mathrm{H}_{3} \mathrm{PO}_{4}$. The following abbreviations and their combinations are used: br, broad; s, singlet; $d$, doublet; $t$, triplet; q, quartet; $\mathrm{m}$, multiplet. ${ }^{1} \mathrm{H}$ and ${ }^{13} \mathrm{C}$ resonance signals were attributed by means of 2D COSY, HSQC and HMBC experiments. Mass spectra were recorded on Hewlett Packard 5989A spectrometer. All commercially available reagents were used without further purification otherwise noted.

Synthesis of 1: $\mathrm{PR}_{2}=\mathrm{P}(\mathrm{N} t \mathrm{Bu})_{2} \mathrm{SiMe}_{2}$. To a stirred solution of imine $^{20}(10.0 \mathrm{~g}, 37.1 \mathrm{mmol})$ in $80 \mathrm{~mL}$ of THF at $-78^{\circ} \mathrm{C}$ was added $n$ butyllithium $(1.6 \mathrm{M}, 24.3 \mathrm{~mL}, 38.9 \mathrm{mmol})$ and the mixture was allowed to warm up to room temperature over $1 \mathrm{~h}$ under stirring. The solution was cooled to $-78{ }^{\circ} \mathrm{C}$ and chlorophosphine $(9.9 \mathrm{~g}, 37.1 \mathrm{mmol})$ was added. The mixture was allowed to warm up to room temperature and the solvent was removed under vacuum. The solid was washed with acetonitrile $(3 \times 20 \mathrm{~mL})$, dried and dissolved with pentane, and then filtered. The volatile substances were removed under vacuum, and compound 1 (17.4 g, $94 \%$ ) was obtained as a white solid. Spectroscopic data of $\mathbf{1}$ are in perfect agreement with the values already reported. ${ }^{20} \mathrm{PR}_{2}=\mathrm{PPh}_{2}$. To a solution of imine $(10.00 \mathrm{~g}, 37.1 \mathrm{mmol})$ in THF $(80 \mathrm{~mL})$ was added dropwise at $-80{ }^{\circ} \mathrm{C}$ a solution of $n$-butyllithium $1.6 \mathrm{M}$ in hexane $(23.2 \mathrm{~mL}, 37.1 \mathrm{mmol})$ and stirred for $1 \mathrm{~h}$. The reaction mixture was warmed up to room temperature and stirred for 1 $\mathrm{h}$, then cooled again to $-80^{\circ} \mathrm{C}$ and chlorodiphenylphosphine $(6.84 \mathrm{~mL}$, $37.1 \mathrm{mmol}$ ) was added dropwise. The resulting solution was warmed up to room temperature and stirred for $1 \mathrm{~h}$, then the solvent was removed under vacuum. Product 1 was extracted with pentane. After evaporation of the solvent, the solid was washed with a minimum of acetonitrile to give $\mathbf{1}$ as a white powder (11.08 g, $66 \%$ yield). Spectroscopic data of $\mathbf{1}$ are in perfect agreement with the values already reported. $^{21}$

Synthesis of $2 a\left(\mathbf{R}^{1}=\mathbf{H} ; \mathbf{R}^{2}=\mathbf{R}^{3}=\mathbf{M e}\right)$ : To a solution of iminophosphine $\mathbf{1}(2.00 \mathrm{~g}, 4.0 \mathrm{mmol})$ in THF $(25 \mathrm{~mL})$ was added dropwise at $-80{ }^{\circ} \mathrm{C}$ a solution of $n$-butyllithium $1.6 \mathrm{M}$ in hexane $(2.5 \mathrm{~mL}, 4.0$ $\mathrm{mmol}$ ) and stirred for $1 \mathrm{~h}$. The reaction mixture was warmed up to room temperature and stirred for $1 \mathrm{~h}$, then cooled again to $-80{ }^{\circ} \mathrm{C}$ and chlorodimethylsilane $(0.44 \mathrm{~mL}, 4.0 \mathrm{mmol})$ was added dropwise. The resulting solution was warmed up to room temperature and stirred for $1 \mathrm{~h}$, then the solvent was removed under vacuum. Product 2a was extracted with heptane from the crude and pale-yellow crystals were obtained from a saturated heptane solution at $-30{ }^{\circ} \mathrm{C}(1.20 \mathrm{~g}, 54 \%$ yield $) .{ }^{1} \mathrm{H}$ NMR (300 MHz, $\left.\mathrm{C}_{6} \mathrm{D}_{6}\right): \delta=7.15-7.01$ (m, 3H, $\mathrm{CH}_{\text {dipp }}$ ), 5.55 (dsept., $\left.{ }^{2} J_{\mathrm{PH}}=15.3 \mathrm{~Hz},{ }^{3} J_{\mathrm{H}-\mathrm{H}}=2.7 \mathrm{~Hz}, 1 \mathrm{H}, \mathrm{SiH}\right), 3.86\left(\mathrm{~s}, 1 \mathrm{H}, \mathrm{C} H_{\text {bridgehead }}\right), 3.43$ (sept., ${ }^{3} J_{\mathrm{HH}}=6.9 \mathrm{~Hz}, 1 \mathrm{H}, \mathrm{CH}_{\mathrm{iPr}}$ ), 3.32 (sept., ${ }^{3} \mathrm{~J}_{\mathrm{HH}}=6.8 \mathrm{~Hz}, 1 \mathrm{H}, \mathrm{C} H_{\mathrm{iPr}}$ ), $2.56\left(\mathrm{~s}, 1 \mathrm{H}, \mathrm{C} H_{\text {bridgehead }}\right), 1.98-1.71\left(\mathrm{~m}, 2 \mathrm{H}, \mathrm{CH}_{2}\right), 1.58-1.47(\mathrm{~m}, 1 \mathrm{H}$, $\left.\mathrm{CH}_{2}\right), 1.47-1.38\left(\mathrm{~m}, 2 \mathrm{H}, \mathrm{CH}_{2}\right), 1.34\left(\mathrm{~s}, 9 \mathrm{H}, \mathrm{CH}_{3 \mathrm{tBu}}\right), 1.32(\mathrm{~s}, 9 \mathrm{H}$, $\left.\mathrm{CH}_{3 \mathrm{tBu}}\right), 1.28-1.16\left(\mathrm{~m}, 12 \mathrm{H}, \mathrm{CH}_{3 \mathrm{PPr}}\right), 1.10-1.03\left(\mathrm{~m}, 2 \mathrm{H}, \mathrm{CH}_{2}\right), 0.56(\mathrm{dd}$, $\left.{ }^{3} J_{\mathrm{HH}}=2.8 \mathrm{~Hz},{ }^{3} \mathrm{JH}_{\mathrm{PH}}=4.4 \mathrm{~Hz}, 3 \mathrm{H}, \mathrm{SiH}-\mathrm{CH}_{3}\right), 0.50\left(\mathrm{~s}, 6 \mathrm{H}, \mathrm{Si}-\mathrm{CH}_{3}\right), 0.14$ $\left(\mathrm{dd},{ }^{3} \mathrm{~J}_{\mathrm{HH}}=3.2 \mathrm{~Hz},{ }^{3} J_{\mathrm{PH}}=1.9 \mathrm{~Hz}, 3 \mathrm{H}, \mathrm{SiH}-\mathrm{CH}_{3}\right) .{ }^{13} \mathrm{C} \mathrm{NMR}(75 \mathrm{MHz}$, $\left.\mathrm{C}_{6} \mathrm{D}_{6}\right): \delta=163.9\left(\mathrm{~d},{ }^{2} J_{\mathrm{CP}}=26.5 \mathrm{~Hz}, \mathrm{~N}-C\right), 147.9\left(\mathrm{~s}, C_{\mathrm{dipp}}\right), 147.0(\mathrm{~s}$, $C_{\text {dipp }}$ ), 142.3 (s, N- $C_{\text {dipp }}$ ), 127.4 (s, $\left.C \mathrm{H}_{\text {dipp }}\right), 124.4$ (s, $\left.C \mathrm{H}_{\text {dipp }}\right), 124.2$ (s, $\left.C \mathrm{H}_{\text {dipp }}\right), 120.1\left(\mathrm{~d},{ }^{1} J_{\mathrm{CP}}=56.5 \mathrm{~Hz}, C\right.$-P $), 50.9\left(\mathrm{~d},{ }^{2} J_{\mathrm{CP}}=13.7 \mathrm{~Hz}, C_{\mathrm{tBu}}\right)$, $50.7\left(\mathrm{~d},{ }^{2} J_{\mathrm{CP}}=11.3 \mathrm{~Hz}, C_{\mathrm{tBu}}\right), 48.1\left(\mathrm{~d},{ }^{2} J_{\mathrm{CP}}=3.5 \mathrm{~Hz}, C \mathrm{H}_{\text {bridgehead }}\right), 47.0$ (s, $\left.\mathrm{CH}_{2}\right), 42.9$ (s, $\left.\mathrm{CH}_{\text {bridgehead }}\right), 32.7\left(\mathrm{~d},{ }^{3} \mathrm{~J}_{\mathrm{CP}}=4.9 \mathrm{~Hz}, \mathrm{CH}_{3 \mathrm{tBu}}\right), 32.5(\mathrm{~d}$, $\left.{ }^{3} J_{\mathrm{CP}}=5.2 \mathrm{~Hz}, C \mathrm{H}_{3 \mathrm{tBu}}\right), 28.8\left(\mathrm{~s}, \mathrm{CH}_{2}\right), 27.9\left(\mathrm{~s}, 2 \mathrm{CH}_{\mathrm{PPr}}\right), 26.8\left(\mathrm{~s}, \mathrm{CH}_{2}\right)$, $25.3\left(\mathrm{~s}, \mathrm{CH}_{3 \mathrm{PPr}}\right), 25.1\left(\mathrm{~s}, C \mathrm{H}_{3 \mathrm{PPr}}\right), 24.8\left(\mathrm{~s}, C \mathrm{H}_{3 \mathrm{iPr}}\right), 24.6\left(\mathrm{~s}, C \mathrm{H}_{3 \mathrm{PPr}}\right), 8.0$ $\left(\mathrm{d},{ }^{3} J_{\mathrm{CP}}=5.3 \mathrm{~Hz}, \mathrm{Si}-\mathrm{CH}_{3}\right), 6.3\left(\mathrm{~s}, \mathrm{Si}-\mathrm{CH}_{3}\right), 0.3\left(\mathrm{~d},{ }^{2} J_{\mathrm{CP}}=24.5 \mathrm{~Hz}, \mathrm{SiH}-\right.$ $\left.\mathrm{CH}_{3}\right), 0.0\left(\mathrm{~d},{ }^{2} J_{\mathrm{CP}}=12.5 \mathrm{~Hz}, \mathrm{SiH}-\mathrm{CH}_{3}\right) .{ }^{31} \mathrm{P}$ NMR $\left(121 \mathrm{MHz}, \mathrm{C}_{6} \mathrm{D}_{6}\right): \delta$ $=114.5\left(\mathrm{~d},{ }^{2} J_{\mathrm{PH}}=15.3 \mathrm{~Hz}\right) \cdot{ }^{29} \mathrm{Si} \mathrm{NMR}\left(60 \mathrm{MHz}, \mathrm{C}_{6} \mathrm{D}_{6}\right): \delta=8.1(\mathrm{~s}$, $\left.\mathrm{SiMe}_{2}\right),-9.2\left(\mathrm{~d},{ }^{1} \mathrm{~J}_{\mathrm{SiP}}=15.8 \mathrm{~Hz}, \mathrm{SiMe}_{2} \mathrm{H}\right)$. M.p. $=209^{\circ} \mathrm{C}$.

Synthesis of $2 b\left(R^{1}=\mathbf{C l} ; \mathbf{R}^{2}=\mathbf{R}^{3}=\mathrm{Me}\right)$ : To a solution of iminophosphine $1\left(\mathrm{PR}_{2}=\right.$ cyclic diaminophosphine $)(2.00 \mathrm{~g}, 4.0 \mathrm{mmol})$ in THF $(20 \mathrm{~mL})$ was added dropwise at $-80{ }^{\circ} \mathrm{C}$ a solution of $n$-butyllithium $1.6 \mathrm{M}$ in hexane $(2.5 \mathrm{~mL}, 4.0 \mathrm{mmol})$ and stirred for $1 \mathrm{~h}$. The reaction mixture was warmed up to room temperature and stirred for $1 \mathrm{~h}$, then cooled again to $-80^{\circ} \mathrm{C}$ and dichlorodimethylsilane $(0.48 \mathrm{~mL}, 4.0$ mmol) was added dropwise. The resulting solution was warmed up to room temperature and stirred for $1 \mathrm{~h}$, then the solvent was removed under vacuum. Product $\mathbf{2 b}$ was extracted with heptane and colorless crystals were obtained from a saturated heptane solution at $-30{ }^{\circ} \mathrm{C}(1.45$ g, $61 \%$ yield). ${ }^{1} \mathrm{H}$ NMR $\left(400 \mathrm{MHz}, \mathrm{CDCl}_{3}, 328 \mathrm{~K}\right): \delta=7.31-7.25(\mathrm{~m}$, $\left.1 \mathrm{H}, \mathrm{C} H_{\text {dipp }}\right), 7.20-7.14$ (m, 2H, $\left.\mathrm{CH}_{\text {dipp }}\right), 3.77$ (s, $\left.1 \mathrm{H}, \mathrm{CH}_{\text {bridgehead }}\right), 3.40$ (sept., ${ }^{3} J_{\mathrm{HH}}=6.8 \mathrm{~Hz}, 1 \mathrm{H}, \mathrm{C} H_{\mathrm{iPr}}$ ), 3.25 (sept., ${ }^{3} J_{\mathrm{HH}}=6.8 \mathrm{~Hz}, 1 \mathrm{H}, \mathrm{C} H_{\mathrm{iPr}}$ ), 2.51 (s, $\left.1 \mathrm{H}, \mathrm{C} H_{\text {bridgehead }}\right), 1.84-1.65\left(\mathrm{~m}, 2 \mathrm{H}, \mathrm{CH}_{2}\right), 1.52-1.36(\mathrm{~m}, 3 \mathrm{H}$, $\left.\mathrm{CH}_{2}\right), 1.35-1.26\left(\mathrm{~m}, 6 \mathrm{H}, \mathrm{CH}_{3 \mathrm{iPr}}\right), 1.31\left(\mathrm{~s}, 9 \mathrm{H}, \mathrm{CH}_{3 \mathrm{tBu}}\right), 1.28(\mathrm{~s}, 9 \mathrm{H}$, $\left.\mathrm{CH}_{3 \mathrm{tBu}}\right), 1.25\left(\mathrm{~d},{ }^{3} J_{\mathrm{HH}}=6.8 \mathrm{~Hz}, 12 \mathrm{H}, \mathrm{CH}_{3 \mathrm{PPr}}\right), 1.24\left(\mathrm{~d},{ }^{3} J_{\mathrm{HH}}=6.8 \mathrm{~Hz}\right.$, $\left.12 \mathrm{H}, \mathrm{CH}_{3 \mathrm{PPr}}\right), 1.06-1.01\left(\mathrm{~m}, 1 \mathrm{H}, \mathrm{CH}_{2}\right), 0.72$ (br, $\left.6 \mathrm{H}, \mathrm{SiCl}-\mathrm{CH}_{3}\right), 0.51$ (s, $\left.3 \mathrm{H}, \mathrm{Si}-\mathrm{CH}_{3}\right), 0.47$ (s, 3H, Si-CH3). ${ }^{13} \mathrm{C}$ NMR (101 MHz, $\mathrm{CDCl}_{3}, 328$ $\mathrm{K}): \delta=161.4\left(\mathrm{~d},{ }^{2} J_{\mathrm{CP}}=26.0 \mathrm{~Hz}, \mathrm{~N}-C\right), 148.4$ (s, $\left.C_{\text {dipp }}\right), 147.4$ (s, $\left.C_{\text {dipp }}\right)$, 142.1 (s, N- $\left.C_{\text {dipp }}\right), 127.5$ (s, $\left.C \mathrm{H}_{\text {dipp }}\right), 124.4$ (s, $\left.C \mathrm{H}_{\text {dipp }}\right), 124.2$ (s, $C \mathrm{H}_{\text {dipp }}$ ), $121.2\left(\mathrm{~d},{ }^{1} J_{\mathrm{CP}}=58.5 \mathrm{~Hz}, C-\mathrm{P}\right), 50.9\left(\mathrm{~d},{ }^{2} J_{\mathrm{CP}}=13.6 \mathrm{~Hz}, C_{\mathrm{tBu}}\right), 50.7(\mathrm{~d}$, $\left.{ }^{2} J_{\mathrm{CP}}=11.5 \mathrm{~Hz}, C_{\mathrm{tBu}}\right), 48.6\left(\mathrm{~d},{ }^{2} J_{\mathrm{CP}}=3.2 \mathrm{~Hz}, C \mathrm{H}_{\text {bridgehead }}\right), 46.7\left(\mathrm{~s}, \mathrm{CH}_{2}\right)$, 42.8 (s, $C \mathrm{H}_{\text {bridgehead }}, 32.6\left(\mathrm{~d},{ }^{3} J_{\mathrm{CP}}=4.6 \mathrm{~Hz}, \mathrm{CH}_{3 \mathrm{tBu}}\right), 32.2\left(\mathrm{~d},{ }^{3} J_{\mathrm{CP}}=5.3\right.$ $\left.\mathrm{Hz}, C \mathrm{H}_{3 \mathrm{tBu}}\right), 28.2\left(\mathrm{~s}, C \mathrm{H}_{2}\right), 27.9\left(\mathrm{~s}, C \mathrm{H}_{\mathrm{iPr}}\right), 27.7\left(\mathrm{~s}, C \mathrm{H}_{\mathrm{iPr}}\right), 26.8\left(\mathrm{~s}, C \mathrm{H}_{2}\right)$, 25.5 (s, $\left.C_{3 \mathrm{PPr}}\right), 25.2$ (s, $\left.\mathrm{CH}_{3 \mathrm{PPr}}\right), 24.9$ (s, $\left.C \mathrm{H}_{3 \mathrm{iPr}}\right), 24.9$ (s, $\left.C_{3 \mathrm{iPr}}\right), 8.0$ $\left(\mathrm{d},{ }^{3} J_{\mathrm{CP}}=5.3 \mathrm{~Hz}, \mathrm{Si}-\mathrm{CH}_{3}\right), 6.3\left(\mathrm{~s}, \mathrm{Si}-\mathrm{CH}_{3}\right), 6.1\left(\mathrm{br} \mathrm{d},{ }^{2} J_{\mathrm{CP}}=22.0 \mathrm{~Hz}, 2\right.$ $\left.\mathrm{SiCl}-\mathrm{CH}_{3}\right) .{ }^{31} \mathrm{P}$ NMR $\left(162 \mathrm{MHz}, \mathrm{CDCl}_{3}, 328 \mathrm{~K}\right): \delta=114.0$ (br). ${ }^{29} \mathrm{Si}$ NMR ( $80 \mathrm{MHz}, \mathrm{CDCl}_{3}, 328 \mathrm{~K}$ ): $\delta=10.3\left(\mathrm{br}, \mathrm{SiMe}_{2} \mathrm{Cl}\right), 9.1$ (s, SiMe 2 ).

Synthesis of $2 c\left(\mathbf{R}^{1}=\mathbf{R}^{2}=\mathbf{C l} ; \mathbf{R}^{3}=\mathbf{M e}\right)$ : To a solution of iminophosphine $1\left(\mathrm{PR}_{2}=\right.$ cyclic diaminophosphine $)(2.00 \mathrm{~g}, 4.0 \mathrm{mmol})$ in THF $(20 \mathrm{~mL})$ was added dropwise at $-80^{\circ} \mathrm{C}$ a solution of $n$-butyllithium $1.6 \mathrm{M}$ in hexane $(2.5 \mathrm{~mL}, 4.0 \mathrm{mmol})$ and stirred for $1 \mathrm{~h}$. The crude was warmed up at room temperature and stirred for $1 \mathrm{~h}$, then cooled to $-80{ }^{\circ} \mathrm{C}$ and trichloromethylsilane $(0.47 \mathrm{~mL}, 4.0 \mathrm{mmol})$ was added dropwise. The resulting solution was warmed up to room temperature and stirred for $1 \mathrm{~h}$, then the solvent was removed under vacuum. Product $\mathbf{2 c}$ was extracted with heptane and colorless crystals were obtained from a saturated heptane solution at $-30{ }^{\circ} \mathrm{C}(1.76 \mathrm{~g}, 72 \%$ yield $) .{ }^{1} \mathrm{H}$ NMR $(500$ $\left.\mathrm{MHz}, \mathrm{CD}_{2} \mathrm{Cl}_{2}\right): \delta=7.33-7.27\left(\mathrm{~m}, 1 \mathrm{H}, \mathrm{CH}_{\text {dipp }}\right), 7.20-7.14(\mathrm{~m}, 2 \mathrm{H}$, $\mathrm{C}_{\text {dipp }}$ ), 3.73 (s, $1 \mathrm{H}, \mathrm{C} H_{\text {bridgehead) }}, 3.31$ (sept., ${ }^{3} J_{\mathrm{HH}}=6.7 \mathrm{~Hz}, 1 \mathrm{H}, \mathrm{C} H_{\mathrm{iPr}}$ ), 3.18 (sept., $\left.{ }^{3} \mathrm{~J}_{\mathrm{HH}}=6.8 \mathrm{~Hz}, 1 \mathrm{H}, \mathrm{CH}_{\mathrm{iPr}}\right), 2.43$ (s, $1 \mathrm{H}, \mathrm{C} H_{\text {bridgehead }}$ ), $1.80-$ $1.63\left(\mathrm{~m}, 2 \mathrm{H}, \mathrm{CH}_{2}\right), 1.47-1.31\left(\mathrm{~m}, 3 \mathrm{H}, \mathrm{CH}_{2}\right), 1.28\left(\mathrm{~d},{ }^{3} \mathrm{~J}_{\mathrm{HH}}=6.8 \mathrm{~Hz}, 3 \mathrm{H}\right.$, $\left.\mathrm{CH}_{3 \mathrm{iPr}}\right), 1.26\left(\mathrm{~s}, 9 \mathrm{H}, \mathrm{CH}_{3 \mathrm{tBu}}\right), 1.25-1.18\left(\mathrm{~m}, 3 \mathrm{x} \mathrm{3H}, \mathrm{CH}_{3 \mathrm{iPr}}\right.$ overlapped by $\mathrm{CH}_{3 \mathrm{tBu}}$ ), 1.23 (s, $9 \mathrm{H}, \mathrm{CH}_{3 \mathrm{tBu}}$ ), 1.07 (br s, $3 \mathrm{H}, \mathrm{SiCl}_{2}-\mathrm{CH}_{3}$ ), $1.03-1.00$ $\left(\mathrm{m}, 1 \mathrm{H}, \mathrm{CH}_{2}\right), 0.48$ (s, 3H, Si-CH3), 0.44 (s, 3H, Si-CH3). ${ }^{13} \mathrm{C}$ NMR $\left(126 \mathrm{MHz}, \mathrm{CD}_{2} \mathrm{Cl}_{2}\right): \delta=159.6\left(\mathrm{~d},{ }^{2} J_{\mathrm{CP}}=25.8 \mathrm{~Hz}, \mathrm{~N}-C\right), 148.9$ (s, $\left.C_{\text {dipp }}\right)$, 147.7 (s, $\left.C_{\text {dipp }}\right), 140.9$ (s, N- $C_{\text {dipp }}$ ), 128.3 (s, $C \mathrm{H}_{\text {dipp }}$ ), 124.7 (s, $C \mathrm{H}_{\text {dipp }}$ ), 124.6 (s, $\left.C \mathrm{H}_{\text {dipp }}\right), 123.8\left(\mathrm{~d},{ }^{1} J_{\mathrm{CP}}=56.5 \mathrm{~Hz}, C\right.$-P), $51.1\left(\mathrm{~d},{ }^{2} J_{\mathrm{CP}}=13.4\right.$ $\left.\mathrm{Hz}, C_{\mathrm{tBu}}\right), 50.9\left(\mathrm{~d},{ }^{2} J_{\mathrm{CP}}=11.6 \mathrm{~Hz}, C_{\mathrm{tBu}}\right), 48.6\left(\mathrm{~d},{ }^{2} J_{\mathrm{CP}}=2.9 \mathrm{~Hz}, C H_{\text {bridge- }}\right.$ head), 47.1 (s, $\left.\mathrm{CH}_{2}\right), 43.1$ (s, $\mathrm{CH}_{\text {bridgehead }}, 32.6$ (d, ${ }^{3} J_{\mathrm{CP}}=4.5 \mathrm{~Hz}, \mathrm{CH}_{3 \mathrm{tBu}}$ ), $32.2\left(\mathrm{~d},{ }^{3} J_{\mathrm{CP}}=5.1 \mathrm{~Hz}, \mathrm{CH}_{3 \mathrm{tBu}}\right), 28.4\left(\mathrm{~s}, 2 \mathrm{CH}_{\mathrm{iPr}}\right), 28.2\left(\mathrm{~s}, \mathrm{CH}_{2}\right), 28.1(\mathrm{~s}$, $\left.2 \mathrm{CH}_{\mathrm{iPr}}\right), 26.9\left(\mathrm{~s}, \mathrm{CH}_{2}\right), 25.7\left(\mathrm{~s}, \mathrm{CH}_{3 \mathrm{iPr}}\right), 25.3\left(\mathrm{~s}, \mathrm{CH}_{3 \mathrm{PPr}}\right), 24.7\left(\mathrm{~s}, \mathrm{CH}_{3 \mathrm{iPr}}\right)$, $24.6\left(\mathrm{~s}, C_{3 \mathrm{iPr}}\right), 9.7\left(\mathrm{~d},{ }^{2} J_{\mathrm{CP}}=24.9 \mathrm{~Hz}, \mathrm{SiCl}-\mathrm{CH}_{3}\right), 7.9\left(\mathrm{~d},{ }^{3} J_{\mathrm{CP}}=5.2 \mathrm{~Hz}\right.$, $\left.\mathrm{Si}-\mathrm{CH}_{3}\right), 6.2\left(\mathrm{~s}, \mathrm{Si}-\mathrm{CH}_{3}\right) .{ }^{31} \mathrm{P}$ NMR $\left(202 \mathrm{MHz}, \mathrm{CD}_{2} \mathrm{Cl}_{2}\right): \delta=111.3$ (s). ${ }^{29} \mathrm{Si} \mathrm{NMR}\left(99 \mathrm{MHz}, \mathrm{CD}_{2} \mathrm{Cl}_{2}\right): \delta=10.1\left(\mathrm{~s}, \mathrm{SiMe}_{2}\right),-5.5\left(\mathrm{~s}, \mathrm{SiMeCl}_{2}\right)$.

Synthesis of $2 \mathrm{~d}\left(\mathbf{R}^{1}=\mathbf{R}^{2}=\mathbf{C l} ; \mathbf{R}^{3}=\mathbf{H} ; \mathbf{P R}_{\mathbf{2}}=\right.$ cyclic diaminophosphine): Intense yellow solid (1.728 g, $72 \%)$. Spectroscopic data are in perfect agreement with the values already reported. ${ }^{10 a}$

Synthesis of $2 \mathbf{e}\left(\mathbf{R}^{1}=\mathbf{R}^{2}=\mathbf{R}^{3}=\mathbf{C l}\right)$ : Orange crystals $(8.6 \mathrm{~g}, 66 \%)$. Spectroscopic data are in perfect agreement with the values already reported. $^{22}$

Synthesis of_2f $\left(\mathbf{R}^{1}=\mathbf{C l} ; \mathbf{R}^{2}=\mathbf{R}^{3}=\mathbf{M e}\right)$ : To a solution of iminophosphine $1\left(\mathrm{PR}_{2}=\mathrm{PPh}_{2}\right)(1.01 \mathrm{~g}, 2.2 \mathrm{mmol})$ in THF $(25 \mathrm{~mL})$ was added dropwise at $-80^{\circ} \mathrm{C}$ a solution of $n$-butyllithium $1.6 \mathrm{M}$ in hexane $(1.4 \mathrm{~mL}, 2.2 \mathrm{mmol})$ and stirred for $1 \mathrm{~h}$. The reaction mixture is then warmed up to room temperature and stirred for $1 \mathrm{~h}$, then cooled again to $-80{ }^{\circ} \mathrm{C}$ and dichlorodimethylsilane $(0.27 \mathrm{~mL}, 2.2 \mathrm{mmol})$ was added 
dropwise. The resulting solution was warmed up to room temperature and stirred for $1 \mathrm{~h}$ before removal of the solvent under vacuum. Product 2f was extracted with heptane and colourless crystals were obtained from a saturated heptane solution at $-30{ }^{\circ} \mathrm{C}(1.11 \mathrm{~g}, 88 \%$ yield $) .{ }^{1} \mathrm{H}$ NMR (300 MHz, $\left.\mathrm{CD}_{2} \mathrm{Cl}_{2}\right): \delta=7.45-6.97\left(\mathrm{~m}, 13 \mathrm{H}, \mathrm{P}\left(\mathrm{C}_{6} H_{5}\right)_{2}+3 \mathrm{C} H_{\text {dipp }}\right)$,

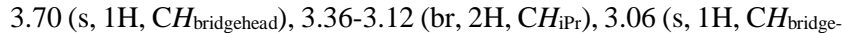
head), $1.89-1.71\left(\mathrm{~m}, 1 \mathrm{H}+1 \mathrm{H}, \mathrm{CH}_{2}\right), 1.60-1.42\left(\mathrm{~m}, 2 \mathrm{H}, \mathrm{CH}_{2}\right), 1.35-1.28$ $\left(\mathrm{m}, 1 \mathrm{H}, \mathrm{CH}_{2}\right), 1.25\left(\mathrm{~d},{ }^{3} J_{\mathrm{HH}}=6.8 \mathrm{~Hz}, 3 \mathrm{H}, \mathrm{CH}_{3 \mathrm{iPr}}\right), 1.15\left(\mathrm{~d},{ }^{3} J_{\mathrm{HH}}=6.8\right.$ $\left.\mathrm{Hz}, 3 \mathrm{H}, \mathrm{CH}_{3 \mathrm{iPr}}\right), 1.04\left(\mathrm{~d},{ }^{3} \mathrm{~J}_{\mathrm{HH}}=6.8 \mathrm{~Hz}, 3 \mathrm{H}, \mathrm{CH} \mathrm{H}_{3 \mathrm{PPr}}\right), 0.82-0.20(\mathrm{br}, 6 \mathrm{H}$ $\left.+1 \mathrm{H}, 2 \mathrm{Si}-\mathrm{CH}_{3}+\mathrm{CH}_{2}\right), 0.74\left(\mathrm{~d},{ }^{3} \mathrm{~J}_{\mathrm{HH}}=6.9 \mathrm{~Hz}, 3 \mathrm{H}, \mathrm{CH}_{3 \mathrm{iPr}}\right) .{ }^{13} \mathrm{C} \mathrm{NMR}$ $\left(75 \mathrm{MHz}, \mathrm{CD}_{2} \mathrm{Cl}_{2}\right): \delta=165.0\left(\mathrm{~d},{ }^{2} J_{\mathrm{CP}}=21.2 \mathrm{~Hz}, \mathrm{~N}-C\right), 148.8$ (s, $C_{\mathrm{dipp}}$ ), 148.7 (s, $\left.C_{\text {dipp }}\right), 146.2$ (s, $\left.C_{\text {dipp }}\right), 146.2$ (s, $\left.C_{\text {dipp }}\right), 143.7\left(\mathrm{~d},{ }^{1} J_{\mathrm{CP}}=12.5\right.$ $\left.\mathrm{Hz}, \mathrm{P}-C_{\mathrm{Ph}}\right), 140.4\left(\mathrm{~d},{ }^{4} J_{\mathrm{CP}}=3.6 \mathrm{~Hz} \mathrm{~N}-C_{\text {dipp }}\right), 138.6\left(\mathrm{~d},{ }^{1} J_{\mathrm{CP}}=17.4 \mathrm{~Hz}, \mathrm{P}-\right.$ $\left.C_{\mathrm{Ph}}\right), 136.8\left(\mathrm{~d}, J_{\mathrm{CP}}=25.2 \mathrm{~Hz}, C \mathrm{H}_{\mathrm{Ph}}\right), 131.6\left(\mathrm{~d}, J_{\mathrm{CP}}=15.1 \mathrm{~Hz}, C \mathrm{H}_{\mathrm{Ph}}\right)$, 129.1 (s, $C \mathrm{H}_{\mathrm{Ph}}$ ), 128.2 (s, $\left.C \mathrm{H}_{\mathrm{Ph}}\right), 127.9$ (d, $\left.J_{\mathrm{CP}}=2.5 \mathrm{~Hz}, C \mathrm{H}_{\mathrm{Ph}}\right), 127.8$ $\left(\mathrm{d}, J_{\mathrm{CP}}=3.0 \mathrm{~Hz}, C \mathrm{H}_{\mathrm{Ph}}\right), 126.3\left(\mathrm{~s}, C \mathrm{H}_{\mathrm{dipp}}\right), 125.4\left(\mathrm{~s}, C \mathrm{H}_{\mathrm{dipp}}\right), 124.6$ (s, $\left.C \mathrm{H}_{\text {dipp }}\right), 107.2\left(\mathrm{~d},{ }^{1} J_{\mathrm{CP}}=21.5 \mathrm{~Hz}, C\right.$-P), $49.1\left(\mathrm{~d},{ }^{3} J_{\mathrm{CP}}=5.5 \mathrm{~Hz}, C \mathrm{H}_{\text {bridge- }}\right.$ head), $47.2\left(\mathrm{~s}, C \mathrm{CH}_{2}\right), 47.0\left(\mathrm{~d},{ }^{3} J_{\mathrm{CP}}=5.2 \mathrm{~Hz}, C \mathrm{H}_{\text {bridgehead }}\right), 28.5\left(\mathrm{~s}, C \mathrm{H}_{\mathrm{PPr}}\right)$, 28.2 (s, $\left.C \mathrm{H}_{\mathrm{PPr}}\right), 27.7\left(\mathrm{~s}, C \mathrm{H}_{2}\right), 27.6\left(\mathrm{~s}, \mathrm{CH}_{2}\right), 25.9$ (s, $\left.C_{3 \mathrm{iPr}}\right), 25.0(\mathrm{~s}$, $\left.C_{3 \mathrm{PPr}}\right), 24.9\left(\mathrm{~s}, \mathrm{CH}_{3 \mathrm{PPr}}\right), 24.6\left(\mathrm{~d}, J_{\mathrm{CP}}=5.8 \mathrm{~Hz}, C_{3 \mathrm{PPr}}\right), 5.1\left(\mathrm{br}, \mathrm{Si}-\mathrm{CH}_{3}\right)$. ${ }^{31} \mathrm{P} \mathrm{NMR}\left(121 \mathrm{MHz}, \mathrm{CD}_{2} \mathrm{Cl}_{2}\right): \delta=-25.4 .{ }^{29} \mathrm{Si} \mathrm{NMR}\left(60 \mathrm{MHz}, \mathrm{CD}_{2} \mathrm{Cl}_{2}\right)$ : $\delta=11.5$. M.p. $=101.1^{\circ} \mathrm{C}$.

Synthesis of $2 \mathrm{~g}\left(\mathbf{R}^{1}=\mathbf{R}^{2}=\mathbf{C l} ; \mathbf{R}^{3}=\mathrm{Me}\right)$ : To a solution of iminophosphine $1\left(\mathrm{PR}_{2}=\mathrm{PPh}_{2}\right)(2.94 \mathrm{~g}, 6.5 \mathrm{mmol})$ in THF $(25 \mathrm{~mL})$ was added dropwise at $-80{ }^{\circ} \mathrm{C}$ a solution of $n$-butyllithium $1.6 \mathrm{M}$ in hexane $(4.14 \mathrm{~mL}, 6.5 \mathrm{mmol})$ and stirred for $1 \mathrm{~h}$. The reaction mixture is then warmed up to room temperature and stirred for $1 \mathrm{~h}$, then cooled again to $-80{ }^{\circ} \mathrm{C}$ and trichloromethylsilane $(0.78 \mathrm{~mL}, 6.5 \mathrm{mmol})$ was added dropwise. The resulting solution was warmed up to room temperature and stirred for $1 \mathrm{~h}$ before removal of the solvent under vacuum. Product $\mathbf{2 g}$ was extracted with heptane and pale-yellow crystals were obtained from a saturated heptane solution at $-30{ }^{\circ} \mathrm{C}(2.48 \mathrm{~g}, 67 \%$ yield $) .{ }^{1} \mathrm{H}$ NMR (300 MHz, $\left.\left.\mathrm{C}_{6} \mathrm{D}_{6}\right): \delta=7.43-6.90\left(\mathrm{~m}, 13 \mathrm{H}, \mathrm{P}\left(\mathrm{C}_{6} H_{5}\right)_{2}\right)+3 \mathrm{C} H_{\text {dipp }}\right)$, $3.91\left(\mathrm{~s}, 1 \mathrm{H}, \mathrm{C} H_{\text {bridgehead }}\right), 3.42$ (sept., $\left.{ }^{3} J_{\mathrm{HH}}=6.7 \mathrm{~Hz}, 1 \mathrm{H}, \mathrm{C} H_{\mathrm{iPr}}\right), 3.28$ (sept., $\left.{ }^{3} J_{\mathrm{HH}}=6.9 \mathrm{~Hz}, 1 \mathrm{H}, \mathrm{C} H_{\mathrm{iPr}}\right), 3.08\left(\mathrm{~s}, 1 \mathrm{H}, \mathrm{CH}_{\text {bridgehead }}\right), 1.91-1.76(\mathrm{~m}$, $\left.1 \mathrm{H}, \mathrm{CH}_{2}\right), 1.75-1.50\left(\mathrm{~m}, 2 \mathrm{H}, \mathrm{CH}_{2}\right), 1.45-1.30\left(\mathrm{~m}, 1 \mathrm{H}, \mathrm{CH}_{2}\right), 1.24(\mathrm{~d}$, $\left.{ }^{3} J_{\mathrm{HH}}=6.7 \mathrm{~Hz}, 3 \mathrm{H}, \mathrm{CH}_{3 \mathrm{iPr}}\right), 1.17\left(\mathrm{~d},{ }^{3} J_{\mathrm{HH}}=6.7 \mathrm{~Hz}, 3 \mathrm{H}, \mathrm{CH}_{3 \mathrm{PPr}}\right), 1.09(\mathrm{~d}$, $\left.{ }^{3} J_{\mathrm{HH}}=6.9 \mathrm{~Hz}, 3 \mathrm{H}, \mathrm{CH}_{3 \mathrm{iPr}}\right), 0.95-0.80\left(\mathrm{~m}, 1 \mathrm{H}, \mathrm{CH}_{2}\right), 0.78\left(\mathrm{~d},{ }^{3} J_{\mathrm{HH}}=6.9\right.$ $\left.\mathrm{Hz}, 3 \mathrm{H}, \mathrm{CH}_{3 \mathrm{iPr}}\right), 0.44$ (s, 3H, Si-CH3). ${ }^{13} \mathrm{C}$ NMR $\left(75 \mathrm{MHz}, \mathrm{C}_{6} \mathrm{D}_{6}\right): \delta=$ $162.7\left(\mathrm{~d},{ }^{2} J_{\mathrm{CP}}=20.7 \mathrm{~Hz}, \mathrm{~N}-C\right), 149.2\left(\mathrm{~d},{ }^{5} J_{\mathrm{CP}}=6.2 \mathrm{~Hz}, C_{\text {dipp }}\right), 146.2(\mathrm{~d}$, $\left.{ }^{5} J_{\mathrm{CP}}=2.2 \mathrm{~Hz}, C_{\mathrm{dipp}}\right), 143.4\left(\mathrm{~d},{ }^{1} J_{\mathrm{CP}}=12.4 \mathrm{~Hz}, \mathrm{P}-C_{\mathrm{Ph}}\right), 138.9\left(\mathrm{~d},{ }^{4} J_{\mathrm{CP}}=\right.$ $3.8 \mathrm{~Hz} C-\mathrm{N}), 138.1\left(\mathrm{~d},{ }^{1} J_{\mathrm{CP}}=17.6 \mathrm{~Hz}, \mathrm{P}-C_{\mathrm{Ph}}\right), 136.9\left(\mathrm{~d}, J_{\mathrm{CP}}=25.4 \mathrm{~Hz}\right.$, $C \mathrm{HPh}_{\mathrm{Ph}}, 131.7\left(\mathrm{~d}, J_{\mathrm{CP}}=15.1 \mathrm{~Hz}, C \mathrm{HPh}_{\mathrm{Ph}}\right), 129.3\left(\mathrm{~s}, C \mathrm{H}_{\mathrm{Ph}}\right), 129.1\left(\mathrm{~s}, C \mathrm{H}_{\mathrm{Ph}}\right)$, $128.0\left(\mathrm{~d}, J_{\mathrm{CP}}=6.1 \mathrm{~Hz}, C \mathrm{H}_{\mathrm{Ph}}\right)$ overlapped by deuterated solvent), 127.9 $\left(\mathrm{d}, J_{\mathrm{CP}}=11.9 \mathrm{~Hz}, C \mathrm{HPh}_{\mathrm{Ph}}\right.$ overlapped by deuterated solvent), 126.5 (s, $\left.C \mathrm{H}_{\text {dipp }}\right), 125.4$ (s, $\left.C \mathrm{H}_{\text {dipp }}\right), 125.1$ (s, $\left.C \mathrm{H}_{\text {dipp }}\right), 111.1\left(\mathrm{~d},{ }^{1} J_{\mathrm{CP}}=24.0 \mathrm{~Hz}, C-\right.$ P), $49.2\left(\mathrm{~d},{ }^{3} J_{\mathrm{CP}}=4.9 \mathrm{~Hz}, C \mathrm{H}_{\text {bridgehead }}\right), 47.4\left(\mathrm{~s}, C \mathrm{H}_{2}\right), 47.1\left(\mathrm{~d},{ }^{2} J_{\mathrm{CP}}=5.1\right.$ $\left.\mathrm{Hz}, C \mathrm{H}_{\text {bridgehead }}\right), 28.7\left(\mathrm{~s}, C \mathrm{H}_{\mathrm{iPr}}\right), 28.2\left(\mathrm{~s}, C \mathrm{H}_{\mathrm{iPr}}\right), 27.5\left(\mathrm{~s}, C \mathrm{H}_{2}\right), 27.4(\mathrm{~s}$, $\left.C_{2}\right), 26.0\left(\mathrm{~d}, J_{\mathrm{CP}}=6.2 \mathrm{~Hz}, C_{3 \mathrm{Pr}}\right), 25.1\left(\mathrm{~s}, C_{3 \mathrm{Pr}}\right), 24.8\left(\mathrm{~s}, \mathrm{CH}_{3 \mathrm{iPr}}\right)$, $24.6\left(\mathrm{~s}, \mathrm{CH}_{3 \mathrm{PPr}}\right), 6.9\left(\mathrm{~s}, \mathrm{Si}-\mathrm{CH}_{3}\right) .{ }^{31} \mathrm{P} \mathrm{NMR}\left(121 \mathrm{MHz}, \mathrm{C}_{6} \mathrm{D}_{6}\right): \delta=-26.1$. ${ }^{29} \mathrm{Si} \mathrm{NMR}\left(60 \mathrm{MHz}, \mathrm{C}_{6} \mathrm{D}_{6}\right): \delta=-5.0$. M.p. $=94.3{ }^{\circ} \mathrm{C}$.

Synthesis of 3: To a solution of $2 \mathbf{a}(300 \mathrm{mg}, 0.54 \mathrm{mmol})$ in dichloromethane $(4 \mathrm{~mL})$ was added trityl cation $(496 \mathrm{mg}, 0.54 \mathrm{mmol})$, and the reaction mixture turns red immediately. The solution was concentrated and cooled as quickly as possible to $-30{ }^{\circ} \mathrm{C}$, affording 3 as pink crystals $(0.437 \mathrm{~g}, 55 \%$ yield $)$. The product is not stable in solution at room temperature, and therefore we applied low-temperature NMR (213 K) to characterise it. ${ }^{1} \mathrm{H}$ NMR (400 MHz, $\mathrm{CD}_{2} \mathrm{Cl}_{2}, 213 \mathrm{~K}$ ): $\delta=7.44-7.09$ (m, $13 \mathrm{H}, \mathrm{C}\left(\mathrm{C}_{6} \mathrm{H}_{5}\right)_{2}$ and $\left.\mathrm{CH}_{\text {dipp }}\right), 6.95(\mathrm{br}, 2 \mathrm{H}, \mathrm{P}-\mathrm{CH}-\mathrm{CH}=\mathrm{CH}), 6.13(\mathrm{br}$, $2 \mathrm{H}, \mathrm{P}-\mathrm{CH}-\mathrm{C} H=\mathrm{CH}), 5.20\left(\mathrm{~d},{ }^{2} J_{\mathrm{PH}}=36.2 \mathrm{~Hz}, 1 \mathrm{H}, \mathrm{P}-\mathrm{CH}\right), 5.04(\mathrm{br} \mathrm{s}, 1 \mathrm{H}$,

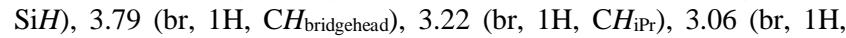
$\left.\mathrm{CH}_{\mathrm{iPr}}\right), 2.96\left(\mathrm{br}, 1 \mathrm{H}, \mathrm{C} H_{\text {bridgehead }}\right), 2.07-1.61\left(\mathrm{br}(\mathrm{m}), 3 \mathrm{H}, 2 \mathrm{CH}_{2}\right), 1.57-$ $0.97\left(\mathrm{~m}, 15 \mathrm{H}, 4 \mathrm{CH}_{3 \mathrm{iPr}}\right.$ and $\left.2 \mathrm{CH}_{2}\right), 1.41\left(\mathrm{~s}, 9 \mathrm{H}, \mathrm{CH}_{3 \mathrm{tBu}}\right), 1.34(\mathrm{~s}, 9 \mathrm{H}$, $\left.\mathrm{CH}_{3 \mathrm{tBu}}\right), 0.77$ (s, 3H, Si-CH $\mathrm{CH}_{3}, 0.76$ (s, 3H, Si-CH3), 0.46 (s, 3H, SiH$\left.\mathrm{CH}_{3}\right),-0.10$ (s, 3H, SiH-CH 3$) .{ }^{13} \mathrm{C}$ NMR $\left(101 \mathrm{MHz}, \mathrm{CD}_{2} \mathrm{Cl}_{2}, 213 \mathrm{~K}\right): \delta$ $=174.0\left(\mathrm{~d},{ }^{2} J_{\mathrm{CP}}=7.1 \mathrm{~Hz}, \mathrm{~N}-C\right), 147.8\left(\mathrm{br} \mathrm{d}, J_{\mathrm{CF}}=240.0 \mathrm{~Hz}, C_{\mathrm{Ar}}-\mathrm{F}\right)$ 147.0 (s, $\left.C_{\text {dipp }}\right), 145.8$ (s, $\left.C_{\text {dipp }}\right), 143.4\left(\mathrm{~d}, J_{C P}=12.7 \mathrm{~Hz}, C(\mathrm{Ph})_{2}\right), 140.7$ $\left(\mathrm{d}, J_{C P}=4.4 \mathrm{~Hz}, C_{\mathrm{Ph}}\right), 140.6\left(\mathrm{~d}, J_{C P}=4.3 \mathrm{~Hz}, C_{\mathrm{Ph}}\right), 138.7$ (s, N- $\left.C_{\text {dipp }}\right)$, $138.2\left(\mathrm{br} \mathrm{d}, J_{\mathrm{CF}}=245.7 \mathrm{~Hz}, C_{A r}-\mathrm{F}\right), 136.1\left(\mathrm{br} \mathrm{d}, J_{\mathrm{CF}}=245.1 \mathrm{~Hz}, \mathrm{C}_{\mathrm{Ar}^{-}}\right.$ F), $131.9\left(\mathrm{~d},{ }^{3} J_{C P}=16.3 \mathrm{~Hz}, \mathrm{P}-\mathrm{CH}-\mathrm{CH}=C \mathrm{H}\right), 131.7\left(\mathrm{~d},{ }^{3} J_{C P}=14.1 \mathrm{~Hz}\right.$, $\mathrm{P}-\mathrm{CH}-\mathrm{CH}=\mathrm{CH}), 130.8\left(\mathrm{~s}, \mathrm{CH}_{\mathrm{Ph}}\right.$ and $\left.\mathrm{CH}_{\text {dipp }}\right), 128.3\left(\mathrm{CH}_{\mathrm{Ph}}\right), 128.2$
$\left(C \mathrm{H}_{\mathrm{Ph}}\right), 125.8\left(\mathrm{~d}, J_{C P}=12.5 \mathrm{~Hz}, C=\mathrm{C}(\mathrm{Ph})_{2}\right), 125.6\left(\mathrm{~s}, C \mathrm{H}_{\mathrm{dipp}}\right), 125.5(\mathrm{~s}$, $C \mathrm{H}_{\text {dipp }}$ ), 123.7 (br, B- $C_{A r}$ ), 120.9 (br, P-CH- $\left.C H=C H\right), 119.4$ (br, P-CH$C \mathrm{H}=\mathrm{CH}), 101.9\left(\mathrm{~d},{ }^{1} J_{\mathrm{CP}}=89.7 \mathrm{~Hz}, C-\mathrm{P}\right), 53.5\left(\mathrm{~d}, C_{\mathrm{tBu}}\right.$ overlapped by deuterated solvant), 53.4 (d, $C_{\mathrm{tBu}}$ overlapped by deuterated solvant), $51.9\left(\mathrm{~d},{ }^{2} J_{\mathrm{CP}}=11.6 \mathrm{~Hz}, C \mathrm{H}_{\text {bridgehead }}\right), 49.1\left(\mathrm{~d},{ }^{2} J_{\mathrm{CP}}=8.6 \mathrm{~Hz}, C \mathrm{H}_{\text {bridgehead }}\right)$, $47.3\left(\mathrm{~d},{ }^{2} J_{\mathrm{CP}}=61.5 \mathrm{~Hz}, \mathrm{P}-\mathrm{CH}\right), 43.8\left(\mathrm{~s}, \mathrm{CH}_{2}\right), 32.3\left(\mathrm{~d}, 2 \mathrm{CH}_{3 \mathrm{tBu}}\right.$ overlapped), 28.4 (s, $\left.C \mathrm{H}_{\mathrm{iPr}}\right), 28.0\left(\mathrm{~s}, \mathrm{CH}_{2}\right), 27.9$ (s, $\left.C \mathrm{H}_{\mathrm{iPr}}\right), 26.9\left(\mathrm{~s}, \mathrm{CH}_{2}\right)$, $25.3\left(\mathrm{~s}, C \mathrm{H}_{3 \mathrm{iPr}}\right), 25.0\left(\mathrm{~s}, C_{3 \mathrm{PPr}}\right), 24.9\left(\mathrm{~s}, C_{3} \mathrm{H}_{3 \mathrm{Pr}}\right), 24.7\left(\mathrm{~s}, C_{3} \mathrm{H}_{3 \mathrm{Pr}}\right), 4.7(\mathrm{~s}$, $\left.\mathrm{Si}-\mathrm{CH}_{3}\right), 3.6\left(\mathrm{~d},{ }^{3} J_{\mathrm{CP}}=2.9 \mathrm{~Hz}, \mathrm{Si}-\mathrm{CH}_{3}\right), 2.8\left(\mathrm{~s}, \mathrm{SiH}-\mathrm{CH}_{3}\right),-3.4(\mathrm{~s}, \mathrm{SiH}-$ $\mathrm{CH}_{3}$ ). ${ }^{31} \mathrm{P}$ NMR $\left(162 \mathrm{MHz}, \mathrm{CD}_{2} \mathrm{Cl}_{2}, 213 \mathrm{~K}\right): \delta=49.3$ (s). ${ }^{19} \mathrm{~F}$ NMR $\left(376 \mathrm{MHz}, \mathrm{CD}_{2} \mathrm{Cl}_{2}\right): \delta=-132.8\left(\mathrm{br}, \mathrm{C}_{\mathrm{Ar}}-F_{\text {ortho }}\right),-163.5\left(\mathrm{t}, J_{\mathrm{FF}}=20.4 \mathrm{~Hz}\right.$, $\left.\mathrm{C}_{\mathrm{Ar}}-F_{\text {para }}\right),-167.3\left(\mathrm{t}, J_{\mathrm{FF}}=18.0 \mathrm{~Hz}, C_{\mathrm{Ar}}-F_{\text {meta }}\right) .{ }^{11} \mathrm{~B} \mathrm{NMR}(128 \mathrm{MHz}$, $\mathrm{CD}_{2} \mathrm{Cl}_{2}, 213 \mathrm{~K}$ ): $\delta=-16.9$ (s, BAr). ${ }^{29} \mathrm{Si} \mathrm{NMR}\left(80 \mathrm{MHz}, \mathrm{CD}_{2} \mathrm{Cl}_{2}, 213\right.$ $\mathrm{K}): \delta=22.3\left(\mathrm{~d},{ }^{2} J_{\mathrm{SiP}}=4.5 \mathrm{~Hz}, \mathrm{SiMe}_{2}\right),-3.7\left(\mathrm{~s}, \mathrm{SiMe}_{2} \mathrm{H}\right)$.

Synthesis of $\mathbf{4 b}\left(\mathbf{R}^{2}=\mathbf{R}^{\mathbf{3}}=\mathbf{M e}\right)$ : To a solution of $\mathbf{2 b}(300 \mathrm{mg}, 0.50$ $\mathrm{mmol})$ in dichloromethane $(3 \mathrm{~mL})$ was added potassium tetrakis(pentafluorophenyl)borane $(0.360,0.50 \mathrm{mmol})$. After stirring for $10 \mathrm{~min}$, the solution was filtered then the solvents are removed under vacuum to give $\mathbf{4 b}$ as a white powder $(0.490 \mathrm{~g}, 79 \%)$. Crystals suitable for XRay diffraction analysis were obtained from a concentrated dichloromethane solution. ${ }^{1} \mathrm{H}$ NMR $\left(300 \mathrm{MHz}, \mathrm{CD}_{2} \mathrm{Cl}_{2}\right)$ : $\delta=7.45-7.24(\mathrm{~m}, 3 \mathrm{H}$, $\mathrm{C}_{\text {dipp }}$ ), 3.39 (s, $1 \mathrm{H}, \mathrm{C} H_{\text {bridgehead), }} 2.96$ (sept., ${ }^{3} J_{\mathrm{HH}}=6.7 \mathrm{~Hz}, 1 \mathrm{H}, \mathrm{CH}_{\mathrm{iPr}}$ ), 2.83 (sept., $\left.{ }^{3} J_{\mathrm{HH}}=6.7 \mathrm{~Hz}, 1 \mathrm{H}, \mathrm{C} H_{\mathrm{iPr}}\right), 2.71\left(\mathrm{~s}, 1 \mathrm{H}, \mathrm{C} H_{\text {bridgehead }}\right), 2.06-$ $1.83\left(\mathrm{~m}, 2 \mathrm{H}, \mathrm{CH}_{2}\right), 1.81-1.66\left(\mathrm{~m}, 2 \mathrm{H}, \mathrm{CH}_{2}\right), 1.61-1.52\left(\mathrm{~m}, 2 \mathrm{H}, \mathrm{CH}_{2}\right)$, $1.40\left(\mathrm{~s}, 9 \mathrm{H}, \mathrm{CH}_{3 \mathrm{tBu}}\right), 1.37\left(\mathrm{~s}, 9 \mathrm{H}, \mathrm{CH}_{3 \mathrm{tBu}}\right), 1.29\left(\mathrm{~d},{ }^{3} \mathrm{~J}_{\mathrm{HH}}=4.0 \mathrm{~Hz}, 3 \mathrm{H}\right.$, $\left.\mathrm{CH}_{3 \mathrm{PPr}}\right), 1.26\left(\mathrm{~d},{ }^{3} J_{\mathrm{HH}}=6.8 \mathrm{~Hz}, 3 \mathrm{H}, \mathrm{CH}_{3 \mathrm{iPr}}\right), 1.18\left(\mathrm{~d},{ }^{3} J_{\mathrm{HH}}=4.0 \mathrm{~Hz}, 3 \mathrm{H}\right.$, $\left.\mathrm{CH}_{3 \mathrm{iPr}}\right), 1.16\left(\mathrm{~d},{ }^{3} J_{\mathrm{HH}}=6.7 \mathrm{~Hz}, 3 \mathrm{H}, \mathrm{CH}_{3 \mathrm{PPr}}\right), 0.82\left(\mathrm{~d},{ }^{3} J_{\mathrm{HP}}=6.6 \mathrm{~Hz}, 3 \mathrm{H}\right.$, $\left.\mathrm{Si}^{+}-\mathrm{CH}_{3}\right), 0.77\left(\mathrm{~s}, 3 \mathrm{H}, \mathrm{Si}-\mathrm{CH}_{3}\right), 0.69\left(\mathrm{~d},{ }^{3} J_{\mathrm{HP}}=6.4 \mathrm{~Hz}, 3 \mathrm{H}, \mathrm{Si}^{+}-\mathrm{CH}_{3}\right)$, 0.65 (s, 3H, Si-CH3). ${ }^{13} \mathrm{C}$ NMR $\left(75 \mathrm{MHz}, \mathrm{CD}_{2} \mathrm{Cl}_{2}\right): \delta=192.3\left(\mathrm{~d},{ }^{2} J_{\mathrm{CP}}=\right.$ $25.9 \mathrm{~Hz}, \mathrm{~N}-C$ ), 148.6 (br d, $\left.J_{\mathrm{CF}}=241.3 \mathrm{~Hz}, C_{\mathrm{Ar}}-\mathrm{F}\right), 147.5$ (s, $\left.C_{\text {dipp }}\right)$, 147.3 (s, $C_{\text {dipp }}$ ), 138.6 (br d, $\left.J_{\mathrm{CF}}=244.8 \mathrm{~Hz}, C_{\mathrm{Ar}}-\mathrm{F}\right), 136.7$ (br d, $J_{\mathrm{CF}}=$ $\left.245.8 \mathrm{~Hz}, C_{\mathrm{Ar}}-\mathrm{F}\right), 132.1\left(\mathrm{~d},{ }^{3} J_{\mathrm{CP}}=3.1 \mathrm{~Hz}, \mathrm{~N}-C_{\text {dipp }}\right), 129.9$ (s, $\left.C \mathrm{H}_{\text {dipp }}\right)$, 125.8 (s, $C \mathrm{H}_{\text {dipp }}$ ), 125.7 (s, $C \mathrm{H}_{\text {dipp }}$ ), 99.0 (d, ${ }^{1} J_{\mathrm{CP}}=68.9 \mathrm{~Hz}, C$-P), 53.3 $\left(\mathrm{d},{ }^{2} J_{\mathrm{CP}}=21.2 \mathrm{~Hz}, C_{\mathrm{tBu}}\right), 53.2\left(\mathrm{~d},{ }^{2} J_{\mathrm{CP}}=19.4 \mathrm{~Hz}, C_{\mathrm{tBu}}\right), 48.7\left(\mathrm{~d},{ }^{3} J_{\mathrm{CP}}=\right.$ $\left.6.0 \mathrm{~Hz}, \mathrm{CH}_{2}\right), 45.0$ (d, $\left.{ }^{2} J_{\mathrm{CP}}=10.5 \mathrm{~Hz}, \mathrm{CH}_{\text {bridgehead }}\right), 41.5$ (s, $\mathrm{CH}_{\text {bridgehead }}$ ), $32.9\left(\mathrm{~d},{ }^{3} J_{\mathrm{CP}}=5.6 \mathrm{~Hz}, C \mathrm{H}_{3 \mathrm{tBu}}\right), 32.6\left(\mathrm{~d},{ }^{3} J_{\mathrm{CP}}=5.6 \mathrm{~Hz}, C \mathrm{H}_{3 \mathrm{tBu}}\right), 28.5(\mathrm{~s}$, $\left.C \mathrm{H}_{\mathrm{iPr}}\right), 28.4\left(\mathrm{~s}, C \mathrm{H}_{\mathrm{iPr}}\right), 28.1\left(\mathrm{~s}, C \mathrm{H}_{2}\right), 26.1\left(\mathrm{~s}, C_{3 \mathrm{PPr}}\right), 25.7\left(\mathrm{~d},{ }^{3} J_{\mathrm{CP}}=1.8\right.$ $\left.\mathrm{Hz}, \mathrm{CH}_{2}\right), 25.3$ (s, $\mathrm{CH}_{3 \mathrm{PPr}}$ ), 24.5 (s, $\mathrm{CH}_{3 \mathrm{iPr}}$ ), 24.4 (s, $\left.C_{3 \mathrm{iPr}}\right), 6.1$ (s, Si$\left.\mathrm{CH}_{3}\right), 4.3\left(\mathrm{~d},{ }^{3} J_{\mathrm{CP}}=1.8 \mathrm{~Hz}, \mathrm{Si}-\mathrm{CH}_{3}\right), 1.4\left(\mathrm{~d},{ }^{2} J_{\mathrm{CP}}=14.2 \mathrm{~Hz}, \mathrm{Si}^{+}-\mathrm{CH}_{3}\right)$, $0.7\left(\mathrm{~d},{ }^{2} J_{\mathrm{CP}}=13.4 \mathrm{~Hz}, \mathrm{Si}^{+}-\mathrm{CH}_{3}\right) . \mathrm{C}_{\mathrm{ipso}}$ attached to boron atom could not be detected. ${ }^{31} \mathrm{P}$ NMR $\left(121 \mathrm{MHz}, \mathrm{CD}_{2} \mathrm{Cl}_{2}\right): \delta=39.6\left({ }^{1} J_{\mathrm{PSi}}=132 \mathrm{~Hz}\right)$. ${ }^{11} \mathrm{~B}$ NMR $\left(96 \mathrm{MHz}, \mathrm{CD}_{2} \mathrm{Cl}_{2}\right): \delta=-16.6(\mathrm{~s}, B \mathrm{Ar}) .{ }^{19} \mathrm{~F}$ NMR $(282 \mathrm{MHz}$, $\left.\mathrm{CD}_{2} \mathrm{Cl}_{2}\right): \delta=-133.0\left(\mathrm{br}, \mathrm{C}_{\mathrm{Ar}}-F_{\text {ortho }}\right),-163.7\left(\mathrm{t}, J_{\mathrm{FF}}=20.3 \mathrm{~Hz}, \mathrm{C}_{\mathrm{Ar}}-F_{\text {para }}\right)$, $-167.5\left(\mathrm{t}, J_{\mathrm{FF}}=18.3 \mathrm{~Hz}, \mathrm{C}_{\mathrm{Ar}}-F_{\text {meta }}\right) .{ }^{29} \mathrm{Si} \mathrm{NMR}\left(60 \mathrm{MHz}, \mathrm{CD}_{2} \mathrm{Cl}_{2}\right): \delta=$ $21.4\left(\mathrm{~d},{ }^{2} J_{\mathrm{SiP}}=2.9 \mathrm{~Hz}, S i \mathrm{Me}_{2}\right), 6.1\left(\mathrm{~d},{ }^{1} J_{\mathrm{SiP}}=132.0 \mathrm{~Hz}, S i^{+}\right)$. Elemental Analysis: Calc. for $\mathrm{C}_{55} \mathrm{H}_{55} \mathrm{BF}_{20} \mathrm{~N}_{3} \mathrm{PSi}_{2}$ : C, 53.45; H, 4.49; N, 3.40 . Found: C, 53.21; H, 4.43; N, 3.38. M.p. $=86.1{ }^{\circ} \mathrm{C}$.

Synthesis of $4 \mathbf{c}\left(\mathbf{R}^{2}=\mathbf{M e}, \mathbf{R}^{3}=\mathbf{C l}\right)$ : White powder $(0.504 \mathrm{~g}, 82 \%)$. Crystals suitable for $\mathrm{X}$-Ray diffraction analysis were obtained from a concentrated dichloromethane solution. A 50-50 diastereomer mixture is obtained. ${ }^{1} \mathrm{H}$ NMR $\left(400 \mathrm{MHz}, \mathrm{CD}_{2} \mathrm{Cl}_{2}\right): \delta=7.47-7.40(\mathrm{~m}, 2 \mathrm{H}$, $\mathrm{CH}_{\text {dipp }}$ ), 7.35-7.26 (m, 4H, $\mathrm{C} H_{\text {dipp }}$ ), 3.43 (s, $2 \mathrm{H}, \mathrm{CH}_{\text {bridgehead), }} 3.07$ (sept., $\left.{ }^{3} J_{\mathrm{HH}}=6.6 \mathrm{~Hz}, 1 \mathrm{H}, \mathrm{C} H_{\mathrm{iPr}}\right), 2.91$ (sept., ${ }^{3} J_{\mathrm{HH}}=6.9 \mathrm{~Hz}, 1 \mathrm{H}, \mathrm{CH}_{\mathrm{iPr}}$ overlapped with $\mathrm{CH}_{\mathrm{iPr}}$ ), 2.89 (sept., ${ }^{3} J_{\mathrm{HH}}=6.9 \mathrm{~Hz}, 1 \mathrm{H}, \mathrm{CH}_{\mathrm{iPr}}$ overlapped with $\left.\mathrm{CH}_{\mathrm{iPr}}\right), 2.83-2.70$ (br, $1 \mathrm{H}, \mathrm{CH}_{\mathrm{iPr}}$ overlapped by $\left.\mathrm{C} H_{\text {bridgehead }}\right), 2.83$ 2.70 (br, $2 \mathrm{H}, \mathrm{CH}_{\text {bridgehead }}$ overlapped by $\left.\mathrm{CH}_{\mathrm{iPr}}\right), 2.05-1.89\left(\mathrm{~m}, 4 \mathrm{H}, \mathrm{CH}_{2}\right)$, 1.81-1.66 (m, 4H, CH2), 1.64-1.56 (m, 2H, CH2), $1.45\left(\mathrm{~d},{ }^{4} \mathrm{~J}_{\mathrm{PH}}=0.88\right.$ $\left.\mathrm{Hz}, 9 \mathrm{H}, \mathrm{CH}_{3 \mathrm{tBu}}\right), 1.44\left(\mathrm{~d},{ }^{4} J_{\mathrm{PH}}=0.87 \mathrm{~Hz}, 3 \mathrm{H}, \mathrm{CH}_{3 \mathrm{tBu}}\right), 1.40\left(\mathrm{~d},{ }^{4} J_{\mathrm{PH}}=\right.$ $\left.0.98 \mathrm{~Hz}, 3 \mathrm{H}, \mathrm{CH} \mathrm{H}_{3 \mathrm{Bu}}\right), 140-1.36\left(\mathrm{~m}, 2 \mathrm{H}, \mathrm{CH}_{2}\right), 1.38\left(\mathrm{~d},{ }^{4} J_{\mathrm{PH}}=0.94 \mathrm{~Hz}\right.$, $\left.3 \mathrm{H}, \mathrm{CH}_{3 \mathrm{tBu}}\right), 1.32-1.26\left(\mathrm{~m}, 12 \mathrm{H}, \mathrm{CH}_{3 \mathrm{PPr}}\right), 1.21-1.14\left(\mathrm{~m}, 12 \mathrm{H}, \mathrm{CH}_{3 \mathrm{iPr}}\right.$ overlapped by $\left.\mathrm{Si}^{+}-\mathrm{CH}_{3}\right), 1.21-1.14\left(\mathrm{~d},{ }^{3} \mathrm{JHH}_{\mathrm{PH}}=4.1 \mathrm{~Hz}^{*}, 3 \mathrm{H}, \mathrm{Si}^{+}-\mathrm{CH}_{3}\right.$ overlapped by $\left.\mathrm{CH}_{3 \mathrm{iPr}}\right), 1.03\left(\mathrm{~d},{ }^{3} \mathrm{~J}_{\mathrm{PH}}=4.1 \mathrm{~Hz}, 3 \mathrm{H}, \mathrm{Si}^{+}-\mathrm{CH}_{3}\right), 0.81(\mathrm{~d}$, $\left.{ }^{2} J_{\mathrm{PH}}=2.2 \mathrm{~Hz}, 6 \mathrm{H}, \mathrm{Si}-\mathrm{CH}_{3}\right), 0.70\left(\mathrm{~d},{ }^{2} J_{\mathrm{PH}}=2.5 \mathrm{~Hz}, 6 \mathrm{H}, \mathrm{Si}-\mathrm{CH}_{3}\right)$. * coupling constant determined by HSQC ${ }^{29} \mathrm{Si}^{1}{ }^{1} \mathrm{H} .{ }^{13} \mathrm{C}$ NMR $(101 \mathrm{MHz}$, $\left.\mathrm{CD}_{2} \mathrm{Cl}_{2}\right): \delta=192.4\left(\mathrm{~d},{ }^{2} J_{\mathrm{CP}}=23.2 \mathrm{~Hz}, \mathrm{~N}-C\right), 192.3\left(\mathrm{~d},{ }^{2} J_{\mathrm{CP}}=23.2 \mathrm{~Hz}\right.$, $\mathrm{N}-C$ ), $148.6\left(\right.$ br d, $\left.J_{\mathrm{CF}}=240.4 \mathrm{~Hz}, \mathrm{C}_{\mathrm{Ar}}-\mathrm{F}\right), 148.1$ (s, $\left.C_{\text {dipp }}\right), 147.9$ (s, $C_{\text {dipp }}$ ), 147.0 (s, $\left.C_{\text {dipp }}\right), 146.7$ (s, $\left.C_{\text {dipp }}\right), 138.6\left(\mathrm{br} \mathrm{d}, J_{\mathrm{CF}}=245.1 \mathrm{~Hz}, \mathrm{C}_{\mathrm{Ar}^{-}}\right.$ F), 136.7 (br d, $\left.J_{\mathrm{CF}}=246.4 \mathrm{~Hz}, \mathrm{C}_{\mathrm{Ar}}-\mathrm{F}\right), 130.7\left(\mathrm{~d},{ }^{3} J_{\mathrm{CP}}=2.8 \mathrm{~Hz}, \mathrm{~N}-C_{\mathrm{dip}}\right)$, $130.6\left(\mathrm{~d},{ }^{3} J_{\mathrm{CP}}=2.6 \mathrm{~Hz}, \mathrm{~N}-C_{\text {dipp }}\right), 130.4\left(\mathrm{~s}, C \mathrm{H}_{\mathrm{dipp}}\right), 130.3$ (s, $\left.C \mathrm{H}_{\text {dipp }}\right)$, 126.3 (s, $C \mathrm{H}_{\text {dipp }}$ ), 126.2 (s, $C \mathrm{H}_{\text {dipp }}$ ), 125.8 (s, $C \mathrm{H}_{\text {dipp }}$ ), 125.7 (s, $C \mathrm{H}_{\text {dipp}}$ ), $124.4\left(\right.$ broad s, B- $\left.C_{A r}\right), 99.3\left(\mathrm{~d},{ }^{1} J_{\mathrm{CP}}=74.6 \mathrm{~Hz}, C-\mathrm{P}\right), 99.2\left(\mathrm{~d},{ }^{1} J_{\mathrm{CP}}=\right.$ 
$72.8 \mathrm{~Hz}, C-\mathrm{P}), 54.4$ (d, $C_{\mathrm{tBu}}$ overlapped by deuterated solvant), 54.2 (d, $\left.{ }^{2} J_{\mathrm{CP}}=1.5 \mathrm{~Hz}, C_{\mathrm{tBu}}\right), 53.7\left(\mathrm{~d},{ }^{2} J_{\mathrm{CP}}=1.2 \mathrm{~Hz}, C_{\mathrm{tBu}}\right), 53.5\left(\mathrm{~d},{ }^{2} J_{\mathrm{CP}}=1.2 \mathrm{~Hz}\right.$ $\left.C_{\mathrm{tBu}}\right), 48.9\left(\mathrm{~d},{ }^{3} J_{\mathrm{CP}}=6.7 \mathrm{~Hz}, \mathrm{CH}_{2}\right), 48.4\left(\mathrm{~d},{ }^{3} J_{\mathrm{CP}}=6.1 \mathrm{~Hz}, \mathrm{CH}_{2}\right), 45.3$

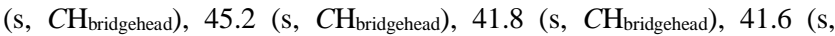
$\left.\mathrm{CH}_{\text {bridgehead }}\right), 32.8\left(\mathrm{~d},{ }^{3} J_{\mathrm{CP}}=5.6 \mathrm{~Hz}, 2 \mathrm{CH}_{3 \mathrm{tBu}}\right), 32.5\left(\mathrm{~d},{ }^{3} J_{\mathrm{CP}}=5.8 \mathrm{~Hz}\right.$, $\left.\mathrm{CH}_{3 \mathrm{tBu}}\right), 32.4\left(\mathrm{~d},{ }^{3} \mathrm{~J}_{\mathrm{CP}}=5.6 \mathrm{~Hz}, \mathrm{CH}_{3 \mathrm{tBu}}\right), 28.8\left(\mathrm{~s}, \mathrm{CH}_{\mathrm{iPr}}\right), 28.7\left(\mathrm{~s}, \mathrm{CH}_{\mathrm{iPr}}\right)$, 28.6 (s, $C \mathrm{H}_{\mathrm{iPr}}$ ), 28.5 (s, $\left.C \mathrm{H}_{\mathrm{iPr}}\right), 27.9$ (s, $\left.C \mathrm{H}_{2}\right), 27.6\left(\mathrm{~s}, \mathrm{CH}_{2}\right), 26.1$ (s, $\left.\mathrm{CH}_{3 \mathrm{iPr}}\right), 25.7\left(\mathrm{~s}, \mathrm{CH}_{3 \mathrm{PPr}}\right), 25.5\left(\mathrm{~d},{ }^{3} J_{\mathrm{CP}}=1.8 \mathrm{~Hz}, \mathrm{CH}_{2}\right), 25.5\left(\mathrm{~d},{ }^{3} J_{\mathrm{CP}}=1.8\right.$ $\left.\mathrm{Hz}, \mathrm{CH}_{2}\right), 25.3\left(\mathrm{~s}, \mathrm{CH}_{3 \mathrm{PPr}}\right), 25.1\left(\mathrm{~s}, \mathrm{CH}_{3 \mathrm{PPr}}\right), 25.0\left(\mathrm{~s}, \mathrm{CH}_{3 \mathrm{iPr}}\right), 24.9$ (s, $\mathrm{CH}_{3 \mathrm{PPr}}$ ), 24.4 (s, $\left.\mathrm{CH}_{3 \mathrm{iPr}}\right), 24.3$ (s, $\mathrm{CH}_{3 \mathrm{PPr}}$ ), 6.0 (s, $\left.\mathrm{Si}-\mathrm{CH}_{3}\right), 5.9$ (s, $2 \mathrm{Si}-$ $\left.\mathrm{CH}_{3}\right), 4.4\left(\mathrm{~d},{ }^{2} J_{\mathrm{CP}}=18.9 \mathrm{~Hz}, \mathrm{Si}^{+}-\mathrm{CH}_{3}\right), 4.3\left(\mathrm{~d},{ }^{3} \mathrm{~J}_{\mathrm{CP}}=1.7 \mathrm{~Hz}, \mathrm{Si}-\mathrm{CH}_{3}\right)$, $4.2\left(\mathrm{~d},{ }^{3} J_{\mathrm{CP}}=1.7 \mathrm{~Hz}, \mathrm{Si}-\mathrm{CH}_{3}\right), 3.7\left(\mathrm{~d},{ }^{2} J_{\mathrm{CP}}=18.0 \mathrm{~Hz}, \mathrm{Si}^{+}-\mathrm{CH}_{3}\right) .{ }^{31} \mathrm{P}$ NMR $\left(162 \mathrm{MHz}, \mathrm{CD}_{2} \mathrm{Cl}_{2}\right): \delta=30.2\left({ }^{1} J_{\mathrm{PSi}}=172.6 \mathrm{~Hz}\right), 30.0\left({ }^{1} J_{\mathrm{PSi}}=\right.$ $171.2 \mathrm{~Hz}) .{ }^{11} \mathrm{~B}$ NMR $\left(160 \mathrm{MHz}, \mathrm{CD}_{2} \mathrm{Cl}_{2}\right): \delta=-16.6$ (s, BAr). ${ }^{19} \mathrm{~F} \mathrm{NMR}$ $\left(471 \mathrm{MHz}, \mathrm{CD}_{2} \mathrm{Cl}_{2}\right): \delta=-132.5\left(\mathrm{br}, \mathrm{C}_{\mathrm{Ar}}-F_{\text {ortho }}\right),-163.3\left(\mathrm{t}, J_{\mathrm{FF}}=20.5 \mathrm{~Hz}\right.$, $\left.\mathrm{C}_{\mathrm{Ar}}-F_{\text {para }}\right),-167.0\left(\mathrm{t}, J_{\mathrm{FF}}=19.3 \mathrm{~Hz}, \mathrm{C}_{\mathrm{Ar}}-F_{\text {meta }}\right) .{ }^{29} \mathrm{Si} \mathrm{NMR}(99 \mathrm{MHz}$ $\left.\mathrm{CD}_{2} \mathrm{Cl}_{2}\right): \delta=23.8\left(\mathrm{~d},{ }^{2} J_{\mathrm{SiP}}=2.5 \mathrm{~Hz}, S i \mathrm{Me}_{2}\right), 23.8\left(\mathrm{~d},{ }^{2} J_{\mathrm{SiP}}=2.7 \mathrm{~Hz}\right.$, $\left.S i \mathrm{Me}_{2}\right),-0.3\left(\mathrm{~d},{ }^{1} J_{\mathrm{SiP}}=172.6 \mathrm{~Hz}, \mathrm{Si}^{+}\right),-2.1\left(\mathrm{~d},{ }^{1} J_{\mathrm{SiP}}=171.2 \mathrm{~Hz}, \mathrm{Si}^{+}\right)$. M.p. $=157.7^{\circ} \mathrm{C}$.

Synthesis of $4 \mathbf{d}\left(\mathbf{R}^{2}=\mathbf{C l}, \mathbf{R}^{3}=\mathbf{H}\right)$ : White powder $(0.511 \mathrm{~g}, 82 \%)$. Crystals suitable for X-Ray diffraction analysis were obtained from a concentrated chloroform solution. A single diastereomer is obtained. ${ }^{1} \mathrm{H}$ NMR $\left(400 \mathrm{MHz}, \mathrm{CDCl}_{3}\right): \delta=7.49-7.43\left(\mathrm{~m}, 1 \mathrm{H}, \mathrm{CH}_{\text {dipp }}\right), 7.33-7.28$ (m, 2H, CH $\left.H_{\text {dipp }}\right), 5.90\left(\mathrm{~d}, 1 \mathrm{H},{ }^{2} J_{\mathrm{PH}}=58.4 \mathrm{~Hz}, \mathrm{Si} H\right), 3.45\left(\mathrm{~s}, 1 \mathrm{H}, \mathrm{C} H_{\text {bridge- }}\right.$ head), 2.96 (sept., ${ }^{3} J_{\mathrm{HH}}=6.9 \mathrm{~Hz}, 1 \mathrm{H}, \mathrm{C} H_{\mathrm{iPr}}$ ), 2.87 (sept., ${ }^{3} J_{\mathrm{HH}}=6.9 \mathrm{~Hz}$, $1 \mathrm{H}, \mathrm{CH}_{\mathrm{iPr}}$ overlapped by $\left.\mathrm{C} H_{\text {bridgehead }}\right), 2.83\left(\mathrm{~s}, 1 \mathrm{H}, \mathrm{C} H_{\text {bridgehead }}\right.$ overlapped by $\left.\mathrm{CH}_{\mathrm{iPr}}\right), 2.07-1.93\left(\mathrm{~m}, 2 \mathrm{H}, \mathrm{CH}_{2}\right), 1.82-1.60\left(\mathrm{~m}, 4 \mathrm{H}, 2 \mathrm{CH}_{2}\right)$, $1.43\left(\mathrm{~d}, 9 \mathrm{H},{ }^{4} J_{\mathrm{PH}}=0.8 \mathrm{~Hz}, \mathrm{CH}_{3 \mathrm{tBu}}\right), 1.41\left(\mathrm{~d}, 9 \mathrm{H},{ }^{4} J_{\mathrm{PH}}=0.8 \mathrm{~Hz}, \mathrm{CH}_{3 \mathrm{tBu}}\right)$, $1.29\left(\mathrm{~d}, 3 \mathrm{H},{ }^{3} J_{\mathrm{HH}}=6.8 \mathrm{~Hz}, \mathrm{CH}_{3 i \mathrm{Pr}}\right), 1.27\left(\mathrm{~d}, 3 \mathrm{H},{ }^{3} J_{\mathrm{HH}}=6.8 \mathrm{~Hz}, \mathrm{CH}_{3 i \mathrm{Pr}}\right)$, $1.24\left(\mathrm{~d}, 6 \mathrm{H},{ }^{3} J_{\mathrm{HH}}=6.8 \mathrm{~Hz}, 2 \mathrm{CH}_{3 i \mathrm{Pr}}\right), 0.80\left(\mathrm{~s}, 3 \mathrm{H}, \mathrm{SiMe}_{2}\right), 0.72(\mathrm{~s}, 3 \mathrm{H}$, $\left.\mathrm{SiMe}_{2}\right) .{ }^{13} \mathrm{C}$ NMR $\left(101 \mathrm{MHz}, \mathrm{CDCl}_{3}\right): \delta=192.3\left(\mathrm{~d},{ }^{2} J_{\mathrm{CP}}=22.2 \mathrm{~Hz}, \mathrm{~N}-\right.$ $C), 148.2\left(\right.$ br d, $\left.J_{\mathrm{CF}}=241.3 \mathrm{~Hz}, \mathrm{C}_{\mathrm{Ar}}-\mathrm{F}\right), 146.7$ (s, $\left.C_{\mathrm{dipp}}\right), 146.6$ (s, $\left.C_{\text {dipp }}\right)$, $138.3\left(\mathrm{br} \mathrm{d}, J_{\mathrm{CF}}=244.9 \mathrm{~Hz}, \mathrm{C}_{\mathrm{Ar}}-\mathrm{F}\right), 136.2\left(\mathrm{br} \mathrm{d}, J_{\mathrm{CF}}=244.1 \mathrm{~Hz}, \mathrm{C}_{\mathrm{Ar}}-\right.$ F), 130.4 (s, $\left.C \mathrm{H}_{\text {dipp }}\right), 130.1$ (s, N- $\left.C_{\text {dipp }}\right), 125.6$ (s, $\left.2 C \mathrm{H}_{\text {dipp }}\right), 100.3$ (d, $\left.{ }^{1} J_{\mathrm{CP}}=76.7 \mathrm{~Hz}, C-\mathrm{P}\right), 53.5\left(\mathrm{br}, C_{\mathrm{tBu}}\right), 53.4\left(\mathrm{br}, C_{\mathrm{tBu}}\right), 48.3\left(\mathrm{~d}, J_{\mathrm{CP}}=6.4\right.$ $\left.\mathrm{Hz}, \mathrm{CH}_{2}\right), 44.8$ (d, $\left.{ }^{3} J_{\mathrm{CP}}=10.0 \mathrm{~Hz}, C \mathrm{H}_{\text {bridgehead }}\right), 41.6$ (s, $C \mathrm{H}_{\text {bridgehead }}$ ), $32.6\left(\mathrm{~d},{ }^{3} J_{\mathrm{CP}}=5.8 \mathrm{~Hz}, \mathrm{CH}_{3 \mathrm{tBu}}\right), 32.4\left(\mathrm{~d},{ }^{3} J_{\mathrm{CP}}=5.8 \mathrm{~Hz}, C \mathrm{H}_{3 \mathrm{tBu}}\right), 28.8(\mathrm{~s}$, $\left.C \mathrm{H}_{\mathrm{PPr}}\right), 28.6\left(\mathrm{~s}, C \mathrm{H}_{\mathrm{iPr}}\right), 27.6\left(\mathrm{~s}, C \mathrm{H}_{2}\right), 25.1\left(\mathrm{~s}, C \mathrm{H}_{3 \mathrm{PPr}}\right), 24.8\left(\mathrm{~d}, J_{\mathrm{CP}}=1.7\right.$ $\left.\mathrm{Hz}, \mathrm{CH}_{2}\right), 24.7\left(\mathrm{~s}, \mathrm{CH}_{3 \mathrm{PPr}}\right), 24.5\left(\mathrm{~s}, C \mathrm{H}_{3 \mathrm{iPr}}\right), 24.2\left(\mathrm{~s}, C \mathrm{H}_{3 \mathrm{PPr}}\right), 5.1(\mathrm{~s}$, $\left.\mathrm{SiMe}_{2}\right), 3.9\left(\mathrm{~d},{ }^{3} J_{\mathrm{CP}}=1.7 \mathrm{~Hz}, \mathrm{SiMe}_{2}\right)$. $\mathrm{C}_{\text {ipso }}$ attached to boron atom could not be detected. ${ }^{31} \mathrm{P}$ NMR $\left(162 \mathrm{MHz}, \mathrm{CDCl}_{3}\right): \delta=28.2\left({ }^{1} J_{\mathrm{SiP}}=169.1\right.$ $\left.\mathrm{Hz},{ }^{2} J_{\mathrm{PH}}=58.5 \mathrm{~Hz}\right) .{ }^{11} \mathrm{~B} \mathrm{NMR}\left(160 \mathrm{MHz}, \mathrm{CDCl}_{3}\right): \delta=-16.6(\mathrm{~s}, \mathrm{BAr})$. ${ }^{19} \mathrm{~F}$ NMR $\left(471 \mathrm{MHz}, \mathrm{CDCl}_{3}\right): \delta=-132.5\left(\mathrm{br}, \mathrm{C}_{\mathrm{Ar}}-F_{\text {ortho }}\right),-163.3\left(\mathrm{t}, J_{\mathrm{FF}}\right.$ $\left.=20.5 \mathrm{~Hz}, \mathrm{C}_{\mathrm{Ar}}-F_{\text {para }}\right),-167.0\left(\mathrm{t}, J_{\mathrm{FF}}=19.3 \mathrm{~Hz}, \mathrm{C}_{\mathrm{Ar}}-F_{\text {meta }}\right) .{ }^{29} \mathrm{Si} \mathrm{NMR}(60$ $\left.\mathrm{MHz}, \mathrm{CDCl}_{3}\right): \delta=24.0\left(\mathrm{~s}, \mathrm{SiMe}_{2}\right),-23.3\left(\mathrm{~d},{ }^{1} J_{\mathrm{SiP}}=169.1 \mathrm{~Hz}, \mathrm{Si}^{+}\right)$. M.p. $=150.3^{\circ} \mathrm{C}$.

Synthesis of $4 \mathbf{e}\left(\mathbf{R}^{2}=\mathbf{R}^{3}=\mathbf{C l}\right)$ : White powder $(0.466 \mathrm{~g}, 77 \%)$. Crystals suitable for X-Ray diffraction analysis were obtained from a concentrated chloroform solution. ${ }^{1} \mathrm{H}$ NMR $\left(300 \mathrm{MHz}, \mathrm{CD}_{2} \mathrm{Cl}_{2}\right): \delta=$ 7.54-7.24 (m, 3H, CH $H_{\text {dipp }}$ ), 3.48 (s, $1 \mathrm{H}, \mathrm{C} H_{\text {bridgehead }}$ ), 3.01 (sept., ${ }^{3} J_{\mathrm{HH}}=$ $6.8 \mathrm{~Hz}, 1 \mathrm{H}, \mathrm{CH}_{\mathrm{iPr}}$ ), 2.93-2.77 (sept., ${ }^{3} J_{\mathrm{HH}}=6.7 \mathrm{~Hz}, 1 \mathrm{H}, \mathrm{C} H_{\mathrm{iPr}}$ overlapped by $\left.\mathrm{CH}_{\text {bridgehead }}\right), 2.88-2.77\left(\mathrm{~s}, 1 \mathrm{H}, \mathrm{CH}_{\text {bridgehead }}\right.$ overlapped by $\left.\mathrm{CH}_{\mathrm{iPr}}\right), 2.12-1.55\left(\mathrm{~m}, 2 \mathrm{H}, \mathrm{CH}_{2}\right), 2.12-1.55\left(\mathrm{~m}, 2 \mathrm{H}, \mathrm{CH}_{2}\right), 2.12-1.55$ $\left(\mathrm{m}, 2 \mathrm{H}, \mathrm{CH}_{2}\right), 1.46$ (s, 9H, CH $\left.\mathrm{CH}_{3 \mathrm{Bu}}\right), 1.45$ (s, 9H, $\left.\mathrm{CH}_{3 \mathrm{tBu}}\right), 1.33$ (d, 3H, $\left.{ }^{3} J_{\mathrm{HH}}=5.5 \mathrm{~Hz}, \mathrm{CH}_{3 i \mathrm{Pr}}\right), 1.31\left(\mathrm{~d}, 3 \mathrm{H},{ }^{3} J_{\mathrm{HH}}=5.5 \mathrm{~Hz}, \mathrm{CH}_{3 i \mathrm{Pr}}\right), 0.80(\mathrm{~d}, 6 \mathrm{H}$, $\left.{ }^{3} J_{\mathrm{HH}}=6.7 \mathrm{~Hz}, \mathrm{CH}_{3 \mathrm{iPr}}\right), 0.85$ (s, 3H, SiMe $), 0.75$ (s, 3H, SiMe 2$) .{ }^{13} \mathrm{C}$ NMR (75 MHz, $\left.\mathrm{CD}_{2} \mathrm{Cl}_{2}\right): \delta=191.7\left(\mathrm{~d},{ }^{2} J_{\mathrm{CP}}=20.2 \mathrm{~Hz}, \mathrm{~N}-\mathrm{C}\right), 148.0(\mathrm{br}$ d, $\left.J_{\mathrm{CF}}=243.6 \mathrm{~Hz}, \mathrm{C}_{\mathrm{Ar}}-\mathrm{F}\right), 147.2\left(\mathrm{~s}, C_{\mathrm{dipp}}\right), 146.9$ (s, $\left.C_{\text {dipp }}\right), 138.3(\mathrm{br} \mathrm{d}$, $\left.J_{\mathrm{CF}}=245.5 \mathrm{~Hz}, \mathrm{C}_{\mathrm{Ar}}-\mathrm{F}\right), 136.3\left(\mathrm{br} \mathrm{d}, J_{\mathrm{CF}}=247.4 \mathrm{~Hz}, \mathrm{C}_{\mathrm{Ar}}-\mathrm{F}\right), 130.3(\mathrm{~s}$, $C \mathrm{H}_{\text {dipp }}$ ), 129.0 (br, N- $C_{\text {dipp }}$ ), 125.8 (s, $C \mathrm{H}_{\text {dipp }}$ ), 125.7 (s, $C \mathrm{H}_{\text {dipp }}$ ), 98.6 (d, ${ }^{1} J_{\mathrm{CP}}=80.1 \mathrm{~Hz}, C$-P), $54.3\left(\mathrm{~d},{ }^{3} J_{\mathrm{CP}}=1.9 \mathrm{~Hz}, C_{\mathrm{tBu}}\right), 54.1\left(\mathrm{~d}, C_{\mathrm{tBu}}\right.$ overlapped by deuterated solvent), $47.9\left(\mathrm{~d}, J_{\mathrm{CP}}=6.8 \mathrm{~Hz}, \mathrm{CH}_{2}\right), 45.3\left(\mathrm{~d},{ }^{2} J_{\mathrm{CP}}\right.$ $\left.=9.6 \mathrm{~Hz}, C H_{\text {bridgehead}}\right), 41.6\left(\mathrm{~d},{ }^{3} J_{\mathrm{CP}}=1.2 \mathrm{~Hz}, C H_{\text {bridgehead }}\right), 32.4\left(\mathrm{~d},{ }^{3} J_{\mathrm{CP}}\right.$ $\left.=5.9 \mathrm{~Hz}, C_{3 \mathrm{tBu}}\right), 31.9\left(\mathrm{~d},{ }^{3} J_{\mathrm{CP}}=5.9 \mathrm{~Hz}, C \mathrm{H}_{3 \mathrm{tBu}}\right), 28.4\left(\mathrm{~s}, C \mathrm{H}_{\mathrm{iPr}}\right), 28.3$ (s, $\left.\mathrm{CH}_{\mathrm{iPr}}\right), 27.1$ (s, $\left.\mathrm{CH}_{2}\right), 25.3$ (s, $\left.\mathrm{CH}_{3 \mathrm{iPr}}\right), 24.9$ (d, $\left.J_{\mathrm{CP}}=1.9 \mathrm{~Hz}, C_{2}\right)$, 24.6 (s, $C \mathrm{H}_{3 \mathrm{iPr}}$ ), 24.4(s, $\left.C \mathrm{H}_{3 \mathrm{iPr}}\right), 24.3\left(\mathrm{~s}, C \mathrm{H}_{3 \mathrm{iPr}}\right), 5.3\left(\mathrm{~s}, \mathrm{SiMe}_{2}\right), 3.7$ (d, $\left.{ }^{3} J_{\mathrm{CP}}=1.4 \mathrm{~Hz}, \mathrm{SiMe}_{2}\right) . \mathrm{C}_{\mathrm{ipso}}$ attached to boron atom could not be detected. ${ }^{31} \mathrm{P}$ NMR $\left(121 \mathrm{MHz}, \mathrm{CD}_{2} \mathrm{Cl}_{2}\right): \delta=21.8\left({ }^{1} J_{\mathrm{SiP}}=237.4 \mathrm{~Hz}\right) .{ }^{11} \mathrm{~B}$ NMR $\left(96 \mathrm{MHz}, \mathrm{CD}_{2} \mathrm{Cl}_{2}\right): \delta=-16.6(\mathrm{~s}, \mathrm{BAr}) .{ }^{19} \mathrm{~F}$ NMR $(282 \mathrm{MHz}$, $\left.\mathrm{CD}_{2} \mathrm{Cl}_{2}\right): \delta=-133.0\left(\mathrm{br}, \mathrm{C}_{\mathrm{Ar}}-F_{\text {ortho }}\right),-163.7\left(\mathrm{t}, J_{\mathrm{FF}}=20.3 \mathrm{~Hz}, \mathrm{C}_{\mathrm{Ar}}-F_{\text {para }}\right)$, $-167.5\left(\mathrm{t}, J_{\mathrm{FF}}=18.3 \mathrm{~Hz}, \mathrm{C}_{\mathrm{Ar}}-F_{\text {meta }}\right) .{ }^{29} \mathrm{Si} \mathrm{NMR}\left(60 \mathrm{MHz}, \mathrm{CD}_{2} \mathrm{Cl}_{2}\right): \delta=$ $25.6\left(\mathrm{~d},{ }^{2} J_{\mathrm{SiP}}=2.88 \mathrm{~Hz}, \mathrm{SiMe}_{2}\right),-21.6\left(\mathrm{~d},{ }^{1} J_{\mathrm{SiP}}=237.4 \mathrm{~Hz}, \mathrm{Si}^{+}\right)$. Elemental Analysis: Calc. for $\mathrm{C}_{53} \mathrm{H}_{49} \mathrm{BCl}_{2} \mathrm{~F}_{20} \mathrm{~N}_{3} \mathrm{PSi}_{2}$ : C, 49.86; H, 3.87; N, 3.29. Found: C, 49.79; H, 3.83; N, 3.35. M.p. $=189.4{ }^{\circ} \mathrm{C}$

Synthesis of $5 \mathbf{f}\left(\mathbf{R}^{2}=\mathbf{R}^{3}=\mathbf{M e}\right)$ : To a solution of $2 \mathbf{f}(136 \mathrm{mg}, 0.25$ $\mathrm{mmol}$ ) in dichloromethane $(2 \mathrm{~mL}$ ) was added potassium tetrakis(pentafluorophenyl)borane (179 $\mathrm{mg}, 0.25 \mathrm{mmol})$. After stirring for $10 \mathrm{~min}$, the solution was filtered and all the volatiles are removed under vacuum to give $\mathbf{5 f}$ as a white powder (213 mg, $72 \%)$. ${ }^{1} \mathrm{H}$ NMR $(300 \mathrm{MHz}$, $\left.\mathrm{CD}_{2} \mathrm{Cl}_{2}\right): \delta=7.83-7.56\left(\mathrm{~m}, 10 \mathrm{H}, \mathrm{P}\left(\mathrm{C}_{6} H_{5}\right)_{2}\right), 7.45-7.20$ (m, 3H, $\left.\mathrm{CH}_{\text {dipp }}\right)$, 3.55 (s, $1 \mathrm{H}, \mathrm{C} H_{\text {bridgehead }}$ ), 3.06 (sept., $\left.{ }^{3} J_{\mathrm{HH}}=6.7 \mathrm{~Hz}, 1 \mathrm{H}, \mathrm{C} H_{\mathrm{iPr}}\right), 2.79$ (s,

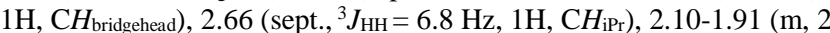
$\left.+1 \mathrm{H}, \mathrm{CH}_{2}\right), 1.67-1.58\left(\mathrm{~m}, 1 \mathrm{H}, \mathrm{CH}_{2}\right), 1.53-1.41\left(\mathrm{~m}, 2 \mathrm{H}, \mathrm{CH}_{2}\right), 1.28(\mathrm{~d}$, $\left.{ }^{3} J_{\mathrm{HH}}=6.7 \mathrm{~Hz}, 3 \mathrm{H}, \mathrm{CH}_{3 \mathrm{iPr}}\right), 1.26\left(\mathrm{~d},{ }^{3} J_{\mathrm{HH}}=6.7 \mathrm{~Hz}, 3 \mathrm{H}, \mathrm{CH}_{3 \mathrm{iPr}}\right), 1.14(\mathrm{~d}$, $\left.{ }^{3} J_{\mathrm{HH}}=6.8 \mathrm{~Hz}, 3 \mathrm{H}, \mathrm{CH}_{3 \mathrm{PPr}}\right), 1.06\left(\mathrm{~d},{ }^{3} J_{\mathrm{HH}}=6.8 \mathrm{~Hz}, 3 \mathrm{H}, \mathrm{CH}_{3 \mathrm{iPr}}\right), 0.81(\mathrm{~d}$, $\left.{ }^{3} J_{\mathrm{PH}}=6.6 \mathrm{~Hz}, 3 \mathrm{H}, \mathrm{Si}-\mathrm{CH}_{3}\right), 0.51\left(\mathrm{~d},{ }^{3} J_{\mathrm{PH}}=6.6 \mathrm{~Hz}, 3 \mathrm{H}, \mathrm{Si}-\mathrm{CH}_{3}\right) \cdot{ }^{13} \mathrm{C}$ $\operatorname{NMR}\left(75 \mathrm{MHz}, \mathrm{CD}_{2} \mathrm{Cl}_{2}\right): \delta=187.5\left(\mathrm{~d},{ }^{2} J_{\mathrm{CP}}=16.4 \mathrm{~Hz}, \mathrm{~N}-\mathrm{C}\right), 148.7(\mathrm{br}$ d, $J_{\mathrm{CF}}=241.3 \mathrm{~Hz}, \mathrm{C}_{\mathrm{Ar}}-\mathrm{F}$ ), 148.0 (s, $\left.C_{\mathrm{dipp}}\right), 147.9$ (s, $\left.C_{\mathrm{dipp}}\right), 138.8$ (br d, $\left.J_{\mathrm{CF}}=244.6 \mathrm{~Hz}, \mathrm{C}_{\mathrm{Ar}}-\mathrm{F}\right), 136.7\left(\mathrm{br} \mathrm{d}, J_{\mathrm{CF}}=247.3 \mathrm{~Hz}, \mathrm{C}_{\mathrm{Ar}}-\mathrm{F}\right), 134.5(\mathrm{~d}$, $\left.J_{\mathrm{CP}}=3.4 \mathrm{~Hz}, C \mathrm{H}_{\mathrm{Ph}}\right), 134.4\left(\mathrm{~d}, J_{\mathrm{CP}}=3.4 \mathrm{~Hz}, C \mathrm{H}_{\mathrm{Ph}}\right), 133.2\left(\mathrm{~d}, J_{\mathrm{CP}}=11.2\right.$ $\left.\mathrm{Hz}, C \mathrm{H}_{\mathrm{Ph}}\right), 132.4\left(\mathrm{~d}, J_{\mathrm{CP}}=11.3 \mathrm{~Hz}, C \mathrm{H}_{\mathrm{Ph}}\right), 131.6\left(\mathrm{~d}, J_{\mathrm{CP}}=2.2 \mathrm{~Hz}, \mathrm{~N}-\right.$ $\left.C_{\text {dipp }}\right), 131.0\left(\mathrm{~d}, J_{\mathrm{CP}}=12.5 \mathrm{~Hz}, C \mathrm{HPh}_{\mathrm{Ph}}, 130.9\left(\mathrm{~d}, J_{C P}=12.7 \mathrm{~Hz}, C \mathrm{HPh}_{\mathrm{Ph}}\right)\right.$, 130.0 (s, $C \mathrm{H}_{\text {dipp }}$ ), 125.8 (s, $\left.C \mathrm{H}_{\text {dipp }}\right), 125.7$ (s, $C \mathrm{H}_{\text {dipp }}$ ), 121.7 (d, ${ }^{1} J_{\mathrm{CP}}=$ $\left.70.3 \mathrm{~Hz}, \mathrm{P}-C_{\mathrm{Ph}}\right), 119.5\left(\mathrm{~d},{ }^{1} J_{\mathrm{CP}}=74.0 \mathrm{~Hz}, \mathrm{P}-C_{\mathrm{Ph}}\right), 86.7\left(\mathrm{~d},{ }^{1} J_{C P}=73.2\right.$ $\mathrm{Hz}, C$-P), $49.3\left(\mathrm{~d},{ }^{3} J_{\mathrm{CP}}=5.0 \mathrm{~Hz}, C \mathrm{H}_{2}\right), 45.1\left(\mathrm{~d},{ }^{2} J_{\mathrm{CP}}=8.9 \mathrm{~Hz}, C \mathrm{H}_{\text {bridge- }}\right.$ head), $42.8\left(\mathrm{~d},{ }^{3} J_{\mathrm{CP}}=1.0 \mathrm{~Hz}, C \mathrm{H}_{\text {bridgehead }}\right), 29.1\left(\mathrm{~s}, \mathrm{CH}_{2}\right), 28.7\left(\mathrm{~s}, C \mathrm{H}_{\mathrm{PPr}}\right)$, $28.6\left(\mathrm{~s}, C_{\mathrm{iPr}}\right), 26.4\left(\mathrm{~d},{ }^{3} J_{\mathrm{CP}}=2.1 \mathrm{~Hz}, C_{2}\right), 25.6\left(\mathrm{~s}, C_{3 \mathrm{PPr}}\right), 25.4(\mathrm{~s}$, $\left.C_{3 \mathrm{PPr}}\right), 24.9\left(\mathrm{~s}, C_{3 \mathrm{iPr}}\right), 23.6\left(\mathrm{~s}, \mathrm{CH}_{3 \mathrm{iPr}}\right),-0.33\left(\mathrm{~d},{ }^{2} J_{\mathrm{CP}}=15.0 \mathrm{~Hz}, \mathrm{Si}^{+}-\right.$ $\left.\mathrm{CH}_{3}\right),-3.0\left(\mathrm{~d},{ }^{2} J_{\mathrm{CP}}=11.0 \mathrm{~Hz}, \mathrm{Si}^{+}-\mathrm{CH}_{3}\right) . \mathrm{C}_{\mathrm{ipso}}$ attached to boron atom could not be detected. ${ }^{31} \mathrm{P}$ NMR $\left(121 \mathrm{MHz}, \mathrm{CD}_{2} \mathrm{Cl}_{2}\right): \delta=-20.8\left({ }^{1} \mathrm{JPSi}_{\mathrm{PS}}=\right.$ $82.1 \mathrm{~Hz}) .{ }^{19} \mathrm{~F}$ NMR $\left(282 \mathrm{MHz}, \mathrm{CD}_{2} \mathrm{Cl}_{2}\right): \delta=-132.9\left(\mathrm{br}, \mathrm{C}_{\mathrm{Ar}}-F_{\text {ortho }}\right)$, $163.6\left(\mathrm{t}, J_{\mathrm{FF}}=20.4 \mathrm{~Hz}, \mathrm{C}_{\mathrm{Ar}}-F_{\text {para }}\right),-167.4\left(\mathrm{t}, J_{\mathrm{FF}}=18.1 \mathrm{~Hz}, \mathrm{C}_{\mathrm{Ar}}-F_{\text {meta }}\right)$. ${ }^{11} \mathrm{~B}$ NMR $\left(96 \mathrm{MHz}, \mathrm{CD}_{2} \mathrm{Cl}_{2}\right): \delta=-16.6$ (s, BAr). ${ }^{29} \mathrm{Si} \mathrm{NMR}(60 \mathrm{MHz}$, $\left.\mathrm{CD}_{2} \mathrm{Cl}_{2}\right): \delta=27.2\left(\mathrm{~d},{ }^{1} J_{\mathrm{PSi}}=82.1 \mathrm{~Hz}\right)$. M.p. $=84.6{ }^{\circ} \mathrm{C}$.

Synthesis of $\mathbf{5 g}\left(\mathbf{R}^{2}=\mathbf{C l} ; \mathbf{R}^{3}=\mathbf{M e}\right)$ : To a solution of $\mathbf{2 g}(300 \mathrm{mg}$, $0.53 \mathrm{mmol})$ in dichloromethane $(2 \mathrm{~mL})$ was added potassium tetrakis(pentafluorophenyl)borane $(380 \mathrm{mg}, 0.53 \mathrm{mmol})$. After stirring for $10 \mathrm{~min}$, the solution was filtered by cannula and evaporated under vacuum to give $\mathbf{5 g}$ as a white powder $(484 \mathrm{mg}, 76 \%$ ). Major isomer (76 \%): ${ }^{1} \mathrm{H}$ NMR $\left(500 \mathrm{MHz}, \mathrm{CD}_{2} \mathrm{Cl}_{2}\right): \delta=7.89-7.60(\mathrm{~m}, 10 \mathrm{H}$, $\left.\mathrm{P}\left(\mathrm{C}_{6} H_{5}\right)_{2}\right), 7.48-7.39$ (m, 1H, $\left.\mathrm{C} H_{\text {dipp }}\right), 7.37-7.29$ (m, $\left.1 \mathrm{H}, \mathrm{C} H_{\text {dipp }}\right), 7.24-$

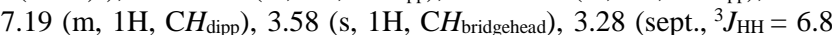
$\left.\mathrm{Hz}, 1 \mathrm{H}, \mathrm{CH}_{\mathrm{iPr}}\right), 2.83$ (s, $\left.1 \mathrm{H}, \mathrm{C} H_{\text {bridgehead }}\right), 2.46$ (sept., ${ }^{3} J_{\mathrm{HH}}=6.7 \mathrm{~Hz}, 1 \mathrm{H}$, $\left.\mathrm{CH}_{\mathrm{iPr}}\right), 2.11-1.91\left(\mathrm{~m}, 2 \mathrm{H}, \mathrm{CH}_{2}\right), 1.68-1.34\left(\mathrm{~m}, 4 \mathrm{H}, 2 \mathrm{CH}_{2}\right), 1.28\left(\mathrm{~d},{ }^{3} J_{\mathrm{HH}}\right.$ $\left.=6.7 \mathrm{~Hz}, 3 \mathrm{H}, \mathrm{CH}_{3 \mathrm{iPr}}\right), 1.27\left(\mathrm{~d},{ }^{3} J_{\mathrm{HH}}=6.7 \mathrm{~Hz}, 3 \mathrm{H}, \mathrm{CH}_{3 \mathrm{PPr}}\right), 1.08\left(\mathrm{~d},{ }^{3} J_{\mathrm{HH}}\right.$ $\left.=6.8 \mathrm{~Hz}, 3 \mathrm{H}, \mathrm{CH}_{3 \mathrm{iPr}}\right), 0.94\left(\mathrm{~d},{ }^{3} J_{\mathrm{HH}}=6.8 \mathrm{~Hz}, 3 \mathrm{H}, \mathrm{C} H_{3 \mathrm{PPr}}\right), 0.83\left(\mathrm{~d},{ }^{3} J_{\mathrm{PH}}\right.$ $\left.=4.3 \mathrm{~Hz}, 3 \mathrm{H}, \mathrm{Si}-\mathrm{CH}_{3}\right) \cdot{ }^{13} \mathrm{C}$ NMR $\left(126 \mathrm{MHz}, \mathrm{CD}_{2} \mathrm{Cl}_{2}\right): \delta=185.9(\mathrm{~d}$, $\left.{ }^{2} J_{\mathrm{CP}}=13.1 \mathrm{~Hz}, \mathrm{~N}-C\right), 148.6\left(\mathrm{br} \mathrm{d}, J_{\mathrm{CF}}=241.7 \mathrm{~Hz}, \mathrm{C}_{\mathrm{Ar}}-\mathrm{F}\right), 148.4(\mathrm{~s}$, $\left.C_{\text {dipp }}\right), 147.5$ (s, $\left.C_{\text {dipp }}\right), 138.7$ (br d, $\left.J_{\mathrm{CF}}=243.8 \mathrm{~Hz}, \mathrm{C}_{\mathrm{Ar}}-\mathrm{F}\right), 136.7$ (br d, $\left.J_{\mathrm{CF}}=243.8 \mathrm{~Hz}, \mathrm{C}_{\mathrm{Ar}}-\mathrm{F}\right), 135.4\left(\mathrm{~d}, J_{\mathrm{CP}}=3.4 \mathrm{~Hz}, C \mathrm{H}_{\mathrm{Ph}}\right), 135.2\left(\mathrm{~d}, J_{\mathrm{CP}}=\right.$ $\left.3.5 \mathrm{~Hz}, C \mathrm{H}_{\mathrm{Ph}}\right), 134.0\left(\mathrm{~d}, J_{\mathrm{CP}}=11.1 \mathrm{~Hz}, C \mathrm{H}_{\mathrm{Ph}}\right), 132.6\left(\mathrm{~d}, J_{\mathrm{CP}}=11.4 \mathrm{~Hz}\right.$, $\left.C \mathrm{H}_{\mathrm{Ph}}\right), 131.5\left(\mathrm{~d}, J_{\mathrm{CP}}=12.8 \mathrm{~Hz}, C \mathrm{H}_{\mathrm{Ph}}\right), 130.9\left(\mathrm{~d}, J_{\mathrm{CP}}=13.3 \mathrm{~Hz}, C \mathrm{H}_{\mathrm{Ph}}\right)$, 130.5 (s, $C \mathrm{H}_{\text {dipp }}$ ), 126.2 (s, $C \mathrm{H}_{\text {dipp }}$ ), 125.6 (s, $\left.C \mathrm{H}_{\text {dipp }}\right), 124.5$ (br, B- $C_{\mathrm{Ar}}$ ), $119.5\left(\mathrm{~d},{ }^{1} J_{\mathrm{CP}}=72.5 \mathrm{~Hz}, \mathrm{P}-C_{\mathrm{Ph}}\right), 116.4\left(\mathrm{~d},{ }^{1} J_{\mathrm{CP}}=77.5 \mathrm{~Hz}, \mathrm{P}-C_{\mathrm{Ph}}\right), 89.2$ $\left(\mathrm{d},{ }^{1} J_{C P}=74.6 \mathrm{~Hz}, C-\mathrm{P}\right), 49.1\left(\mathrm{~d},{ }^{3} J_{\mathrm{CP}}=5.5 \mathrm{~Hz}, C \mathrm{H}_{2}\right), 45.4\left(\mathrm{~d},{ }^{2} J_{\mathrm{CP}}=\right.$ $8.5 \mathrm{~Hz}, C \mathrm{H}_{\text {bridgehead }}, 43.1$ (d, $\left.{ }^{3} J_{\mathrm{CP}}=1.8 \mathrm{~Hz}, C \mathrm{H}_{\text {bridgehead }}\right), 29.0$ (s, 2 $\left.\mathrm{CH}_{\mathrm{iPr}}\right), 28.8\left(\mathrm{~s}, \mathrm{CH}_{2}\right), 26.1\left(\mathrm{~d},{ }^{3} J_{\mathrm{C}}=2.3 \mathrm{~Hz}, \mathrm{CH}_{2}\right), 25.5\left(\mathrm{~s}, C_{3 \mathrm{PPr}}\right), 25.5$ (s, $\left.C_{3} \mathrm{H}_{3 \mathrm{Pr}}\right), 24.8\left(\mathrm{~s}, \mathrm{CH}_{3 \mathrm{PPr}}\right), 23.1\left(\mathrm{~s}, C_{3 \mathrm{iPr}}\right), 0.0\left(\mathrm{~d},{ }^{2} J_{\mathrm{CP}}=15.1 \mathrm{~Hz}, \mathrm{Si}^{+}-\right.$ $\left.\mathrm{CH}_{3}\right) . \mathrm{C}_{\mathrm{ipso}}$ attached to boron atom could not be detected. ${ }^{31} \mathrm{P}$ NMR $(202$ $\left.\mathrm{MHz}, \mathrm{CD}_{2} \mathrm{Cl}_{2}\right): \delta=-24.3\left({ }^{1} \mathrm{JSS}_{\mathrm{PS}}=124.6 \mathrm{~Hz}\right) .{ }^{19} \mathrm{~F} \mathrm{NMR}(471 \mathrm{MHz}$, $\left.\mathrm{CD}_{2} \mathrm{Cl}_{2}\right): \delta=-133.0\left(\mathrm{br}, \mathrm{C}_{\mathrm{Ar}}-F_{\text {ortho }}\right),-163.6\left(\mathrm{t}, J_{\mathrm{FF}}=20.4 \mathrm{~Hz}, \mathrm{C}_{\mathrm{Ar}}-F_{\text {para }}\right)$, $-167.4\left(\mathrm{t}, J_{\mathrm{FF}}=18.1 \mathrm{~Hz}, \mathrm{C}_{\mathrm{Ar}}-F_{\text {meta }}\right) .{ }^{11} \mathrm{~B} \mathrm{NMR}\left(160 \mathrm{MHz}, \mathrm{CD}_{2} \mathrm{Cl}_{2}\right): \delta=$ -16.6 (s, BAr). ${ }^{29} \mathrm{Si}$ NMR $\left(99 \mathrm{MHz}, \mathrm{CD}_{2} \mathrm{Cl}_{2}\right): \delta=15.4\left(\mathrm{~d},{ }^{1} \mathrm{JPSi}_{\mathrm{PS}}=124.6\right.$ $\mathrm{Hz})$. M.p. $=78.8{ }^{\circ} \mathrm{C}$. Minor isomer $(24 \%):{ }^{1} \mathrm{H}$ NMR $(500 \mathrm{MHz}$, $\left.\mathrm{CD}_{2} \mathrm{Cl}_{2}\right): \delta=7.89-7.59\left(\mathrm{~m}, 10 \mathrm{H}, \mathrm{P}\left(\mathrm{C}_{6} H_{5}\right)_{2} *\right), 7.48-7.39(\mathrm{~m}, 1 \mathrm{H}$, $\left.\mathrm{C}_{\text {dipp }}{ }^{*}\right), 7.37-7.29$ (m, $1 \mathrm{H}, \mathrm{CH}_{\text {dipp }}$ *), 7.24-7.19 (m, $1 \mathrm{H}, \mathrm{CH}_{\text {dipp }} *$ ), 3.58 (s, $\left.1 \mathrm{H}, \mathrm{CH}_{\text {bridgehead }}{ }^{*}\right), 2.98$ (sept., ${ }^{3} \mathrm{~J}_{\mathrm{HH}}=6.7 \mathrm{~Hz}, 1 \mathrm{H}, \mathrm{C} H_{\mathrm{iPr}}$ ), 2.89-2.79 (br, $\left.1 \mathrm{H}, \mathrm{CH}_{\mathrm{iPr}}{ }^{*}\right), 2.89-2.79$ (br, $1 \mathrm{H}, \mathrm{CH}_{\text {bridgehead }}$ ), 2.11-1.91 (m, $2 \mathrm{H}$, $\left.\mathrm{CH}_{2} *\right), 1.68-1.34\left(\mathrm{~m}, 4 \mathrm{H}, 2 \mathrm{CH}_{2} *\right), 1.31\left(\mathrm{~d},{ }^{3} J_{\mathrm{HH}}=6.7 \mathrm{~Hz}, 3 \mathrm{H}, \mathrm{CH}_{3 \mathrm{iPr}}\right)$, $1.26\left(\mathrm{~d}, 3 \mathrm{H}, \mathrm{CH}_{3 \mathrm{iPr}} *\right), 1.20\left(\mathrm{~d},{ }^{3} J_{\mathrm{HH}}=6.7 \mathrm{~Hz}, 3 \mathrm{H}, \mathrm{C} H_{3 \mathrm{PPr}}\right), 1.18\left(\mathrm{~d},{ }^{3} J_{\mathrm{PH}}\right.$ $=4.4 \mathrm{~Hz}, 3 \mathrm{H}, \mathrm{Si}-\mathrm{CH}_{3}$ overlapped with $\left.\mathrm{CH}_{3 \mathrm{Pr}}\right), 1.17\left(\mathrm{~d},{ }^{3} J_{\mathrm{HH}}=7.0 \mathrm{~Hz}\right.$, $3 \mathrm{H}, \mathrm{CH}_{3 \mathrm{iPr}}$ overlapped with $\left.\mathrm{Si}-\mathrm{CH}_{3}\right) .{ }^{13} \mathrm{C} \mathrm{NMR}\left(126 \mathrm{MHz}, \mathrm{CD}_{2} \mathrm{Cl}_{2}\right): \delta$ 
$=187.3\left(\mathrm{~d},{ }^{2} J_{\mathrm{CP}}=13.9 \mathrm{~Hz}, \mathrm{~N}-C\right), 148.7\left(\mathrm{br} \mathrm{d}, J_{\mathrm{CF}}=241.7 \mathrm{~Hz}, \mathrm{C}_{\mathrm{Ar}}-\mathrm{F}\right)$ 148.6 (s, $C_{\text {dipp }}$ ), 147.5 (s, $\left.C_{\text {dipp }} *\right), 138.7$ (br d, $J_{\mathrm{CF}}=243.8 \mathrm{~Hz}, \mathrm{C}_{\mathrm{Ar}}-\mathrm{F}$ ) $136.7\left(\mathrm{br} \mathrm{d}, J_{\mathrm{CF}}=243.8 \mathrm{~Hz}, \mathrm{C}_{\mathrm{Ar}}-\mathrm{F}\right), 135.3\left(\mathrm{~d}, J_{\mathrm{CP}}=3.5 \mathrm{~Hz}, C \mathrm{H}_{\mathrm{Ph}}\right), 135.1$ $\left(\mathrm{d}, J_{\mathrm{CP}}=3.4 \mathrm{~Hz}, C \mathrm{HPh}_{\mathrm{Ph}}\right), 133.3\left(\mathrm{~d}, J_{\mathrm{CP}}=11.3 \mathrm{~Hz}, C \mathrm{HPh}\right), 133.1\left(\mathrm{~d}, J_{\mathrm{CP}}=\right.$ $11.3 \mathrm{~Hz}, C \mathrm{H}_{\mathrm{Ph}}$ ), 131.4 (d, $\left.C \mathrm{H}_{\mathrm{Ph}}{ }^{*}\right), 131.0$ (d, $\left.C \mathrm{H}_{\mathrm{Ph}} *\right), 129.9$ (s, $C \mathrm{H}_{\text {dipp }}$ ), 126.2 (s, $C \mathrm{H}_{\text {dipp }}$ ), 125.8 (s, $\left.C \mathrm{H}_{\text {dipp }}\right), 124.5$ (br, B- $\left.C_{\mathrm{Ar}}\right), 118.8$ (d, ${ }^{1} J_{\mathrm{CP}}=$ $\left.76.4 \mathrm{~Hz}, \mathrm{P}-C_{\mathrm{Ph}}\right), 118.3\left(\mathrm{~d},{ }^{1} J_{\mathrm{CP}}=75.2 \mathrm{~Hz}, \mathrm{P}-C_{\mathrm{Ph}}\right), 87.0\left(\mathrm{~d},{ }^{1} J_{C P}=73.7\right.$ $\mathrm{Hz}, C$-P), 49.1 (d, $\left.C \mathrm{H}_{2} *\right), 45.5\left(\mathrm{~d},{ }^{2} J_{\mathrm{CP}}=8.8 \mathrm{~Hz}, C \mathrm{H}_{\text {bridgehead }}\right), 42.8$ (s, $C \mathrm{H}_{\text {bridgehead }}$ ), $28.9\left(\mathrm{~s}, 2 \mathrm{CH}_{\mathrm{iPr}}\right), 28.5\left(\mathrm{~s}, \mathrm{CH}_{2}\right), 26.5\left(\mathrm{~d},{ }^{3} \mathrm{~J}_{\mathrm{C}}=2.5 \mathrm{~Hz}, \mathrm{CH}_{2}\right)$, $25.8\left(\mathrm{~s}, \mathrm{CH}_{3 \mathrm{iPr}}\right), 25.3\left(\mathrm{~s}, \mathrm{CH}_{3 \mathrm{iPr}}\right), 24.6\left(\mathrm{~s}, \mathrm{CH}_{3 \mathrm{iPr}}\right), 24.4\left(\mathrm{~s}, \mathrm{CH}_{3 \mathrm{PPr}}\right), 2.9$ $\left(\mathrm{d},{ }^{2} J_{\mathrm{CP}}=17.8 \mathrm{~Hz}, \mathrm{Si}^{+}-\mathrm{CH}_{3}\right) .{ }^{31} \mathrm{P}$ NMR $\left(202 \mathrm{MHz}, \mathrm{CD}_{2} \mathrm{Cl}_{2}\right): \delta=-24.1$ $\left({ }^{1} J_{\mathrm{PSi}}=117.4\right) .{ }^{19} \mathrm{~F} \mathrm{NMR}\left(471 \mathrm{MHz}, \mathrm{CD}_{2} \mathrm{Cl}_{2}\right): \delta=-132.9\left(\mathrm{br}, \mathrm{C}_{\mathrm{Ar}}-F_{\text {ortho }}\right)$, $-163.6\left(\mathrm{t}, J_{\mathrm{FF}}=20.4 \mathrm{~Hz}, \mathrm{C}_{\mathrm{Ar}}-F_{\text {para }}\right),-167.4\left(\mathrm{t}, J_{\mathrm{FF}}=18.1 \mathrm{~Hz}, \mathrm{C}_{\mathrm{Ar}}-F_{\text {meta }}\right)$. ${ }^{11} \mathrm{~B}$ NMR $\left(160 \mathrm{MHz}, \mathrm{CD}_{2} \mathrm{Cl}_{2}\right): \delta=-16.6(\mathrm{~s}, B \mathrm{Ar}) .{ }^{29} \mathrm{Si} \mathrm{NMR}(99 \mathrm{MHz}$, $\left.\mathrm{CD}_{2} \mathrm{Cl}_{2}\right): \delta=15.3\left(\mathrm{~d},{ }^{1} J_{\mathrm{PSi}}=117.4 \mathrm{~Hz}\right)$. All signals marked with $*$ are overlapped by signals of the major isomer.

Synthesis of 6: In a J. Young NMR tube, to a solution of $\mathbf{5 f}(50.0$ $\mathrm{mg}, 0.042 \mathrm{mmol})$ in dichloromethane $(0.3 \mathrm{~mL})$ was added methyl acrylate $(3.78 \mu \mathrm{L}, 0.042 \mathrm{mmol})$ then the solution is warmed up at $50{ }^{\circ} \mathrm{C}$ for 4 hours. Then solution was evaporated under vacuum and the resulting crude was washed with $3 \times 0.3 \mathrm{~mL}$ of pentane. Product 6 was isolated as a with powder in $60 \%$ yield $(32 \mathrm{mg}) .{ }^{1} \mathrm{H}$ NMR $\left(500 \mathrm{MHz}, \mathrm{CD}_{2} \mathrm{Cl}_{2}\right)$ : $\delta=7.83-7.53\left(\mathrm{~m}, 8 \mathrm{H}, \mathrm{P}\left(\mathrm{C}_{6} H_{5}\right)_{2}\right), 7.43-7.36\left(\mathrm{~m}, 1 \mathrm{H}, \mathrm{C} H_{\text {dipp }}\right), 7.32-7.25$ (m, $2 \mathrm{H}, \mathrm{C} H_{\text {dipp }}$ overlapped by $\left.\left.\mathrm{P}\left(\mathrm{C}_{6} H_{5}\right)_{2}\right)\right), 7.32-7.25\left(\mathrm{~m}, 2 \mathrm{H}, \mathrm{P}\left(\mathrm{C}_{6} H_{5}\right)_{2}\right.$ overlapped by $\left.\mathrm{C}_{\mathrm{dipp}}\right), 3.94\left(\mathrm{ddd}, 1 \mathrm{H},{ }^{3} J_{\mathrm{PH}}=25.3 \mathrm{~Hz},{ }^{3} J_{\mathrm{HH}}=6.2 \mathrm{~Hz}\right.$, $\left.{ }^{3} J_{\mathrm{HH}}=3.2 \mathrm{~Hz}, \mathrm{CH}=C\right), 3.78\left(\mathrm{ddd}, 1 \mathrm{H},{ }^{3} J_{\mathrm{HH}}=17.7 \mathrm{~Hz},{ }^{2} J_{\mathrm{PH}}=14.3 \mathrm{~Hz}\right.$, $\left.{ }^{3} J_{\mathrm{HH}}=3.2 \mathrm{~Hz}, \mathrm{P}-\mathrm{CH}_{2}\right), 3.52\left(\mathrm{ddd}, 1 \mathrm{H},{ }^{3} J_{\mathrm{HH}}=17.7 \mathrm{~Hz},{ }^{2} J_{\mathrm{PH}}=14.3 \mathrm{~Hz}\right.$, $\left.{ }^{3} J_{\mathrm{HH}}=6.2 \mathrm{~Hz}, \mathrm{P}-\mathrm{CH}_{2}\right), 3.44$ (s, $\left.3 \mathrm{H}, \mathrm{O}-\mathrm{CH}_{3}\right), 3.41$ (sept. ${ }^{3} J_{\mathrm{HH}}=6.9 \mathrm{~Hz}$, $\left.1 \mathrm{H}, \mathrm{C} H_{\mathrm{iPr}}\right), 3.11$ (sept. $\left.{ }^{3} \mathrm{~J}_{\mathrm{HH}}=6.8 \mathrm{~Hz}, 1 \mathrm{H}, \mathrm{CH}_{\mathrm{iPr}}\right), 2.93\left(\mathrm{br}, 1 \mathrm{H}, \mathrm{C} H_{\text {bridge- }}\right.$ head), 2.49 (br, $\left.1 \mathrm{H}, \mathrm{C} H_{\text {bridgehead }}\right), 1.99-1.90\left(\mathrm{~m}, 1 \mathrm{H}, \mathrm{CH}_{2}\right), 1.80-1.52(\mathrm{~m}$, $\left.3 \mathrm{H}, \mathrm{CH}_{2}\right), 1.48-1.41\left(\mathrm{~m}, 1 \mathrm{H}, \mathrm{CH}_{2}\right), 1.38\left(\mathrm{~d},{ }^{3} \mathrm{~J}_{\mathrm{PH}}=6.9 \mathrm{~Hz}, 3 \mathrm{H}, \mathrm{CH}_{3 \mathrm{PPr}}\right)$, $1.31\left(\mathrm{~d},{ }^{3} J_{\mathrm{PH}}=6.8 \mathrm{~Hz}, 3 \mathrm{H}, \mathrm{CH}_{3 \mathrm{iPr}}\right), 1.27\left(\mathrm{~d},{ }^{3} J_{\mathrm{PH}}=6.9 \mathrm{~Hz}, 3 \mathrm{H}, \mathrm{CH}_{3 \mathrm{Pr}}\right)$, $1.24\left(\mathrm{~d},{ }^{3} \mathrm{JH}_{\mathrm{PH}}=6.8 \mathrm{~Hz}, 3 \mathrm{H}, \mathrm{CH}_{3 \mathrm{PPr}}\right), 1.11-1.07\left(\mathrm{~m}, 1 \mathrm{H}, \mathrm{CH}_{2}\right), 0.40(\mathrm{~s}$, $\left.3 \mathrm{H}, \mathrm{Si}-\mathrm{CH}_{3}\right),-0.04\left(\mathrm{~s}, \mathrm{Si}-\mathrm{CH}_{3}\right) .{ }^{13} \mathrm{C}$ NMR $\left(126 \mathrm{MHz}, \mathrm{CD}_{2} \mathrm{Cl}_{2}\right): \delta=$ $177.6\left(\mathrm{~d},{ }^{2} J_{\mathrm{CP}}=2.9 \mathrm{~Hz}, \mathrm{~N}-C\right), 156.9\left(\mathrm{~d},{ }^{3} J_{\mathrm{CP}}=5.5 \mathrm{~Hz}, \mathrm{CH}=C-\mathrm{O}\right), 148.5$ (br d, $\left.J_{\mathrm{CF}}=243.7 \mathrm{~Hz}, \mathrm{C}_{\mathrm{Ar}}-\mathrm{F}\right), 147.6$ (s, $\left.C_{\mathrm{dipp}}\right), 146.7$ (s, $\left.C_{\text {dipp }}\right), 138.6(\mathrm{br}$ d, $\left.J_{\mathrm{CF}}=244.4 \mathrm{~Hz}, \mathrm{C}_{\mathrm{Ar}}-\mathrm{F}\right), 137.4$ (s, $\left.C_{\mathrm{Dipp}}\right), 136.6$ (br d, $J_{\mathrm{CF}}=244.4 \mathrm{~Hz}$, $\left.\mathrm{C}_{\mathrm{Ar}}-\mathrm{F}\right), 134.3\left(\mathrm{~d}, J_{\mathrm{CP}}=3.1 \mathrm{~Hz}, C \mathrm{H}_{\mathrm{Ph}}\right), 133.9\left(\mathrm{~d}, J_{\mathrm{CP}}=2.8 \mathrm{~Hz}, C \mathrm{H}_{\mathrm{Ph}}\right)$, $131.9\left(\mathrm{~d}, J_{\mathrm{CP}}=10.2 \mathrm{~Hz}, C \mathrm{HPh}_{\mathrm{Ph}}, 131.3\left(\mathrm{~d}, J_{\mathrm{CP}}=8.7 \mathrm{~Hz}, C \mathrm{HPh}_{\mathrm{Ph}}\right), 130.3(\mathrm{~d}\right.$, $\left.J_{\mathrm{CP}}=11.9 \mathrm{~Hz}, C \mathrm{H}_{\mathrm{Ph}}\right), 130.1\left(\mathrm{~d}, J_{\mathrm{CP}}=13.0 \mathrm{~Hz}, C \mathrm{H}_{\mathrm{Ph}}\right), 129.5\left(\mathrm{~s}, C \mathrm{H}_{\mathrm{dipp}}\right)$, $128.6\left(\mathrm{~d},{ }^{1} J_{\mathrm{CP}}=91.3 \mathrm{~Hz}, \mathrm{P}-C_{\mathrm{Ph}}\right), 125.9$ (s, $\left.C \mathrm{H}_{\text {dipp }}\right), 125.6$ (s, $\left.C \mathrm{H}_{\text {dipp }}\right)$, $123.7\left(\mathrm{~d},{ }^{1} J_{\mathrm{CP}}=89.6 \mathrm{~Hz}, \mathrm{P}-C_{\mathrm{Ph}}\right), 92.2\left(\mathrm{~d},{ }^{1} J_{C P}=73.2 \mathrm{~Hz}, C-\mathrm{P}\right), 67.8(\mathrm{~d}$, $\left.{ }^{2} J_{\mathrm{CP}}=10.6 \mathrm{~Hz}, C \mathrm{H}=\mathrm{C}\right), 56.0\left(\mathrm{~s}, \mathrm{O}-\mathrm{CH}_{3}\right), 52.1\left(\mathrm{~d},{ }^{2} J_{\mathrm{CP}}=12.9 \mathrm{~Hz}\right.$,

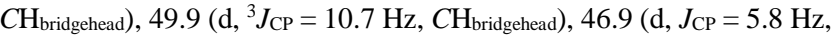
$\mathrm{CH}_{2}$ ), $28.8\left(\mathrm{~s}, \mathrm{CH}_{\mathrm{iPr}}\right), 28.5\left(\mathrm{~s}, \mathrm{CH}_{2}\right), 28.4\left(\mathrm{~s}, \mathrm{CH}_{\mathrm{iPr}}\right), 28.3$ (d, ${ }^{1} J_{\mathrm{CP}}=58.2$ $\left.\mathrm{Hz}, \mathrm{P}-\mathrm{CH}_{2}\right), 26.2\left(\mathrm{~d},{ }^{3} J_{\mathrm{CP}}=2.8 \mathrm{~Hz}, \mathrm{CH}_{2}\right), 25.5\left(\mathrm{~s}, \mathrm{CH}_{3 \mathrm{iPr}}\right), 25.1(\mathrm{~s}$, $\mathrm{CH}_{3 \mathrm{iPr}}$ ), 24.8 (s, $\mathrm{CH}_{3 \mathrm{iPr}}$ ), 23.7 (s, $\mathrm{CH}_{3 \mathrm{PPr}}$ ), -0.8 (s, $\left.\mathrm{Si}-\mathrm{CH}_{3}\right),-1.6$ (s, $\mathrm{Si}-$ $\left.\mathrm{CH}_{3}\right)$. $\mathrm{C}_{\text {ipso }}$ attached to boron atom could not be detected. ${ }^{31} \mathrm{P}$ NMR (202 $\mathrm{MHz}, \mathrm{CD}_{2} \mathrm{Cl}_{2}$ ): $\delta=20.9$ (s). ${ }^{19} \mathrm{~F}$ NMR (471 MHz, $\mathrm{CD}_{2} \mathrm{Cl}_{2}$ ): $\delta=-133.0$ $\left(\mathrm{br}, \mathrm{C}_{\mathrm{Ar}}-F_{\text {ortho }}\right),-163.7\left(\mathrm{t}, J_{\mathrm{FF}}=20.4 \mathrm{~Hz}, \mathrm{C}_{\mathrm{Ar}}-F_{\text {para }}\right),-167.5\left(\mathrm{t}, J_{\mathrm{FF}}=18.1\right.$ $\mathrm{Hz}, m$ of $\left.\mathrm{C}_{\mathrm{Ar}}-F_{\text {meta }}\right) .{ }^{11} \mathrm{~B} \mathrm{NMR}\left(160 \mathrm{MHz}, \mathrm{CD}_{2} \mathrm{Cl}_{2}\right): \delta=-16.6$ (s, BAr). ${ }^{29} \mathrm{Si}$ NMR $\left(99 \mathrm{MHz}, \mathrm{CD}_{2} \mathrm{Cl}_{2}\right): \delta=5.0(\mathrm{~s})$

Synthesis of 7: In a J. Young NMR tube, to a solution of $\mathbf{5 f}(50.0$ $\mathrm{mg}, 0.042 \mathrm{mmol})$ in deuterated dichloromethane $(0.3 \mathrm{~mL})$ was added benzaldehyde $(4.3 \mu \mathrm{L}, 0.042 \mathrm{mmol})$. The reaction immediately proceeds to give adduct 7 in a mixture of 2 diastereomers in a 68:32 ratio. Suitable crystals for X-Ray diffraction were obtained by layering of pentane in dichloromethane. Product 7 was isolated as a white powder in $66 \%$ yield $(36 \mathrm{mg})$. Major isomer $(68 \%)$ : ${ }^{1} \mathrm{H}$ NMR $(500 \mathrm{MHz}$, $\left.\mathrm{CD}_{2} \mathrm{Cl}_{2}\right): \delta=7.91-7.80\left(\mathrm{~m}, 2 \mathrm{H}, \mathrm{CH} H_{\mathrm{Ph}}\right), 7.78-7.68\left(\mathrm{~m}, 2 \mathrm{H}, \mathrm{CH}_{\mathrm{Ph}}\right), 7.68-$ $7.62\left(\mathrm{~m}, 4 \mathrm{H}, \mathrm{C} H_{\mathrm{Ph}}\right), 7.50-7.40\left(\mathrm{~m}, 3 \mathrm{H}, \mathrm{C} H_{\mathrm{Ph}}\right), 7.38-7.28\left(\mathrm{~m}, 3 \mathrm{H}, \mathrm{C} H_{\mathrm{Ph}}\right)$, 7.23-7.15 (m, 2H, CHPh), 6.93-6.87 (m, 2H, CHPh), 6.39 (broad s, $1 \mathrm{H}$, P-CH-O), 3.58 (sept., ${ }^{3} J_{\mathrm{HH}}=6.9 \mathrm{~Hz}, 1 \mathrm{H}, \mathrm{C} H_{\mathrm{iPr}}$ ), 3.20 (sept., ${ }^{3} J_{\mathrm{HH}}=6.7$ $\left.\mathrm{Hz}, 1 \mathrm{H}, 2 \mathrm{CH}_{\mathrm{iPr}}\right), 2.92$ (br, $\left.2 \mathrm{H}, 2 \mathrm{CH}_{\text {bridgehead }}\right), 2.01-1.92\left(\mathrm{~m}, 1 \mathrm{H}, \mathrm{CH}_{2}\right)$, 1.81-1.70 (m, $\left.1 \mathrm{H}, \mathrm{CH}_{2}\right), 1.58-1.48\left(\mathrm{~m}, 2 \mathrm{H}, \mathrm{CH}_{2}\right), 1.40\left(\mathrm{~d},{ }^{3} J_{\mathrm{PH}}=6.9\right.$ $\left.\mathrm{Hz}, 3 \mathrm{H}, \mathrm{CH}_{3 i \mathrm{Pr}}\right), 1.37-1.28\left(\mathrm{~m}, 9 \mathrm{H}, 3 \mathrm{CH}_{3 i \mathrm{Pr}}\right), 1.27-1.23\left(\mathrm{~m}, 1 \mathrm{H}, \mathrm{CH}_{2}\right)$, 1.17-1.10 (m, $\left.1 \mathrm{H}, \mathrm{CH}_{2}\right), 0.67$ (s, $\left.3 \mathrm{H}, \mathrm{Si}-\mathrm{CH}_{3}\right), 0.02\left(\mathrm{~s}, 3 \mathrm{H}, \mathrm{Si}-\mathrm{CH}_{3}\right) .{ }^{13} \mathrm{C}$ NMR $\left(126 \mathrm{MHz}, \mathrm{CD}_{2} \mathrm{Cl}_{2}\right): \delta=177.9\left(\mathrm{~d},{ }^{2} J_{\mathrm{CP}}=5.2 \mathrm{~Hz}, \mathrm{~N}-C\right), 148.6(\mathrm{br}$ $\left.\mathrm{d}, J_{\mathrm{CF}}=243.7 \mathrm{~Hz}, \mathrm{C}_{\mathrm{Ar}}-\mathrm{F}\right), 147.6$ (s, $\left.C_{\text {dipp }}\right), 146.8$ (s, $\left.C_{\text {dipp }}\right), 138.6(\mathrm{br} \mathrm{d}$, $\left.J_{\mathrm{CF}}=244.4 \mathrm{~Hz}, \mathrm{C}_{\mathrm{Ar}}-\mathrm{F}\right), 136.6\left(\mathrm{~s}, \mathrm{~N}-C_{\mathrm{Dipp}}\right), 136.5\left(\mathrm{br} \mathrm{d}, J_{\mathrm{CF}}=244.4 \mathrm{~Hz}\right.$, $\left.\mathrm{C}_{\mathrm{Ar}}-\mathrm{F}\right), 135.8\left(\mathrm{~d}, J_{\mathrm{CP}}=2.8 \mathrm{~Hz}, C \mathrm{H}_{\mathrm{Ph}}\right), 135.7\left(\mathrm{~d}, J_{\mathrm{CP}}=2.8 \mathrm{~Hz}, C \mathrm{H}_{\mathrm{Ph}}\right)$, $134.7\left(\mathrm{~d}, J_{\mathrm{CP}}=9.3 \mathrm{~Hz}, C \mathrm{H}_{\mathrm{Ph}}\right), 133.8\left(\mathrm{~d}, J_{\mathrm{CP}}=9.1 \mathrm{~Hz}, C \mathrm{H}_{\mathrm{Ph}}\right), 131.2(\mathrm{~s}$, $\left.C_{\text {ipso(benzaldehyde })}\right), 130.6\left(\mathrm{~d}, J_{\mathrm{CP}}=12.2 \mathrm{~Hz}, C \mathrm{HPh}_{\mathrm{Ph}}\right), 130.3\left(\mathrm{~d}, J_{\mathrm{CP}}=12.3\right.$ $\left.\mathrm{Hz}, C \mathrm{H}_{\mathrm{Ph}}\right), 129.7$ (d, $\left.J_{\mathrm{CP}}=11.9 \mathrm{~Hz}, C \mathrm{H}_{\mathrm{Ph}}\right), 129.6$ (s, $\left.C \mathrm{H}_{\mathrm{Ph}}\right), 129.1$ (d, $\left.J_{\mathrm{CP}}=2.6 \mathrm{~Hz}, C \mathrm{HPh}\right), 127.9\left(\mathrm{~d}, J_{\mathrm{CP}}=4.8 \mathrm{~Hz}, C \mathrm{HPh}_{\mathrm{Ph}}\right), 126.2\left(\mathrm{~s}, 2 C \mathrm{H}_{\mathrm{dipp}}\right)$, 125.8 (s, $2 C_{\text {dipp }}$ ), 124.4 (br, B- $C_{\mathrm{Ar}}$ ), 118.7 (d, $\left.{ }^{1} J_{\mathrm{CP}}=86.1 \mathrm{~Hz}, \mathrm{P}-C_{\mathrm{Ph}}\right)$, $116.3\left(\mathrm{~d},{ }^{1} J_{\mathrm{CP}}=83.0 \mathrm{~Hz}, \mathrm{P}-C_{\mathrm{Ph}}\right), 90.0\left(\mathrm{~d},{ }^{1} J_{C P}=91.5 \mathrm{~Hz}, C-\mathrm{P}\right), 73.4(\mathrm{~d}$, $\left.{ }^{1} J_{\mathrm{CP}}=62.0 \mathrm{~Hz}, C \mathrm{H}-\mathrm{O}\right), 49.1\left(\mathrm{~d},{ }^{2} J_{\mathrm{CP}}=10.9 \mathrm{~Hz}, C \mathrm{H}_{\text {bridgehead }}\right), 47.1(\mathrm{~d}$, $\left.J_{\mathrm{CP}}=5.7 \mathrm{~Hz}, \mathrm{CH}_{2}\right), 46.6\left(\mathrm{~d},{ }^{2} J_{\mathrm{CP}}=8.4 \mathrm{~Hz}, C \mathrm{H}_{\text {bridgehead }}\right), 28.7\left(\mathrm{~s}, C \mathrm{H}_{\mathrm{iPr}}\right)$, $28.6\left(\mathrm{~s}, C \mathrm{H}_{\mathrm{iPr}}\right), 27.9\left(\mathrm{~s}, C \mathrm{H}_{2}\right), 26.6\left(\mathrm{~d},{ }^{3} J_{\mathrm{CP}}=2.5 \mathrm{~Hz}, C \mathrm{H}_{2}\right), 25.1(\mathrm{~s}$, $\mathrm{CH}_{3 \mathrm{iPr}}$ ), 24.9 (s, $\mathrm{CH}_{3 \mathrm{PPr}}$ ), $24.8\left(\mathrm{~s}, \mathrm{CH}_{3 \mathrm{iPr}}\right), 24.5$ (s, $\left.\mathrm{CH}_{3 \mathrm{PPr}}\right), 0.5$ (s, Si$\left.\mathrm{CH}_{3}\right),-1.4\left(\mathrm{~s}, \mathrm{Si}-\mathrm{CH}_{3}\right) .{ }^{31} \mathrm{P}$ NMR $\left(202 \mathrm{MHz}, \mathrm{CD}_{2} \mathrm{Cl}_{2}\right): \delta=21.5(\mathrm{~s}) .{ }^{19} \mathrm{~F}$ NMR (471 MHz, $\left.\mathrm{CD}_{2} \mathrm{Cl}_{2}\right): \delta=-133.0\left(\mathrm{br}, \mathrm{C}_{\mathrm{Ar}}-F_{\text {ortho }}\right),-163.7\left(\mathrm{t}, J_{\mathrm{FF}}=\right.$ $\left.20.4 \mathrm{~Hz}, \mathrm{C}_{\mathrm{Ar}}-F_{\text {para }}\right),-167.5\left(\mathrm{t}, J_{\mathrm{FF}}=18.1 \mathrm{~Hz}, \mathrm{C}_{\mathrm{Ar}}-F_{\text {meta }}\right) .{ }^{11} \mathrm{~B} \mathrm{NMR}(160$ $\mathrm{MHz}, \mathrm{CD}_{2} \mathrm{Cl}_{2}$ ): $\delta=-16.6$ (s, BAr). ${ }^{29} \mathrm{Si} \mathrm{NMR}\left(99 \mathrm{MHz}, \mathrm{CD}_{2} \mathrm{Cl}_{2}\right): \delta=$ 9.5 (s). Minor isomer (32\%): ${ }^{1} \mathrm{H}$ NMR $\left(500 \mathrm{MHz}, \mathrm{CD}_{2} \mathrm{Cl}_{2}\right): \delta=7.91$ $7.80\left(\mathrm{~m}, 1 \mathrm{H}, \mathrm{C} H_{\mathrm{Ph}}\right), 7.78-7.68\left(\mathrm{~m}, 1 \mathrm{H}, \mathrm{C} H_{\mathrm{Ph}}\right), 7.68-7.62\left(\mathrm{~m}, 4 \mathrm{H}, \mathrm{C} H_{\mathrm{Ph}}\right)$, 7.51-7.40 (m, $\left.3 \mathrm{H}, \mathrm{CH}_{\mathrm{Ph}}\right), 7.38-7.28\left(\mathrm{~m}, 3 \mathrm{H}, \mathrm{C} H_{\mathrm{Ph}}\right), 7.23-7.15(\mathrm{~m}, 2 \mathrm{H}$, $\left.\mathrm{C} H_{\mathrm{Ph}}\right), 7.10-7.03\left(\mathrm{~m}, 2 \mathrm{H}, \mathrm{C} H_{\mathrm{Ph}}\right), 6.85-6.80\left(\mathrm{~m}, 2 \mathrm{H}, \mathrm{C} H_{\mathrm{Ph}}\right), 6.39$ (broad s, $1 \mathrm{H}, \mathrm{P}-\mathrm{CH}-\mathrm{O}$ ), 3.27 (sept., $\left.{ }^{3} J_{\mathrm{HH}}=6.7 \mathrm{~Hz}, 1 \mathrm{H}, \mathrm{C} H_{\mathrm{irr}}\right), 3.20(\mathrm{~m}, 1 \mathrm{H}$, $\left.\mathrm{CH}_{\mathrm{iPr}}\right), 2.92$ (br, $\left.1 \mathrm{H}, \mathrm{CH}_{\text {bridgehead }}\right), 2.81$ (br, $\left.1 \mathrm{H}, \mathrm{CH}_{\text {bridgehead }}\right), 2.01-1.92$ $\left(\mathrm{m}, 1 \mathrm{H}, \mathrm{CH}_{2}\right), 1.92-1.82\left(\mathrm{~m}, 1 \mathrm{H}, \mathrm{CH}_{2}\right), 1.79-1.70\left(\mathrm{~m}, 1 \mathrm{H}, \mathrm{CH}_{2}\right), 1.58-$ $1.48\left(\mathrm{~m}, 1 \mathrm{H}, \mathrm{CH}_{2}\right), 1.37-1.28\left(\mathrm{~m}, 9 \mathrm{H}, 3 \mathrm{CH}_{3 i \mathrm{Pr}}\right), 1.21\left(\mathrm{~d},{ }^{3} J_{\mathrm{PH}}=6.8 \mathrm{~Hz}\right.$, $\left.3 \mathrm{H}, \mathrm{C} H_{3 i \mathrm{Pr}}\right), 1.17-1.10\left(\mathrm{~m}, 1 \mathrm{H}, \mathrm{CH}_{2}\right), 1.03-0.96\left(\mathrm{~m}, 1 \mathrm{H}, \mathrm{CH}_{2}\right), 0.85(\mathrm{~s}$, $\left.3 \mathrm{H}, \mathrm{Si}-\mathrm{CH}_{3}\right), 0.09$ (s, $\left.3 \mathrm{H}, \mathrm{Si}-\mathrm{CH}_{3}\right) .{ }^{13} \mathrm{C} \mathrm{NMR}\left(126 \mathrm{MHz}, \mathrm{CD}_{2} \mathrm{Cl}_{2}\right): \delta=$ $177.7\left(\mathrm{~d},{ }^{2} J_{\mathrm{CP}}=5.2 \mathrm{~Hz}, \mathrm{~N}-C\right), 148.6\left(\mathrm{br} \mathrm{d}, J_{\mathrm{CF}}=243.7 \mathrm{~Hz}, \mathrm{C}_{\mathrm{Ar}}-\mathrm{F}\right), 147.5$ (s, $\left.C_{\text {dipp }}\right), 146.5$ (s, $\left.C_{\text {dipp }}\right), 138.6\left(\mathrm{br} \mathrm{d}, J_{\mathrm{CF}}=244.4 \mathrm{~Hz}, \mathrm{C}_{\mathrm{Ar}}-\mathrm{F}\right), 137.1(\mathrm{~s}$, $\left.C_{\text {Dipp }}\right), 136.5\left(\right.$ br d, $\left.J_{\mathrm{CF}}=244.4 \mathrm{~Hz}, \mathrm{C}_{\mathrm{Ar}}-\mathrm{F}\right), 135.7\left(\mathrm{~d}, J_{\mathrm{CP}}=2.8 \mathrm{~Hz}\right.$, $\left.C \mathrm{H}_{\mathrm{Ph}}\right), 135.4\left(\mathrm{~d}, J_{\mathrm{CP}}=3.0 \mathrm{~Hz}, C \mathrm{H}_{\mathrm{Ph}}\right), 134.8\left(\mathrm{~d}, J_{\mathrm{CP}}=9.8 \mathrm{~Hz}, C \mathrm{H}_{\mathrm{Ph}}\right)$, $133.6\left(\mathrm{~d}, J_{\mathrm{CP}}=9.3 \mathrm{~Hz}, C \mathrm{H}_{\mathrm{Ph}}\right), 132.7$ (s, ipso of $\left.\left(C_{6} \mathrm{H}_{5}\right)_{\text {benzaldehyde }}\right), 131.0$ $\left(\mathrm{d}, J_{\mathrm{CP}}=11.9 \mathrm{~Hz}, C \mathrm{H}_{\mathrm{Ph}}\right), 130.8\left(\mathrm{~d}, J_{\mathrm{CP}}=3.3 \mathrm{~Hz}, C \mathrm{H}_{\mathrm{Ph}}\right), 130.5\left(\mathrm{~d}, J_{\mathrm{CP}}=\right.$ $\left.3.8 \mathrm{~Hz}, C \mathrm{HPh}_{\mathrm{Ph}}\right), 129.5$ (s, $\left.C \mathrm{HPh}_{\mathrm{Ph}}\right), 129.3\left(\mathrm{~d}, J_{\mathrm{CP}}=3.1 \mathrm{~Hz}, C \mathrm{HPh}_{\mathrm{Ph}}\right), 126.9$ (d, $\left.J_{\mathrm{CP}}=4.2 \mathrm{~Hz}, C \mathrm{H}_{\mathrm{Ph}}\right), 125.8\left(\mathrm{~s}, C \mathrm{H}_{\mathrm{dipp}}\right), 125.7$ (s, $\left.C \mathrm{H}_{\text {dipp }}\right), 124.4$ (br, ipso of $\mathrm{B} A r), 119.7\left(\mathrm{~d},{ }^{1} J_{\mathrm{CP}}=82.2 \mathrm{~Hz}, \mathrm{P}-C_{\mathrm{Ph}}\right), 115.2\left(\mathrm{~d},{ }^{1} J_{\mathrm{CP}}=87.4 \mathrm{~Hz}, \mathrm{P}-\right.$ $\left.C_{\mathrm{Ph}}\right), 83.8\left(\mathrm{~d},{ }^{1} J_{C P}=93.5 \mathrm{~Hz}, C-\mathrm{P}\right), 73.5\left(\mathrm{~d},{ }^{1} J_{\mathrm{CP}}=59.6 \mathrm{~Hz}, \mathrm{P}-C \mathrm{H}-\mathrm{O}\right)$, $49.8\left(\mathrm{~d},{ }^{2} J_{\mathrm{CP}}=10.5 \mathrm{~Hz}, C \mathrm{H}_{\text {bridgehead }}\right), 48.0\left(\mathrm{~d},{ }^{2} J_{\mathrm{CP}}=10.4 \mathrm{~Hz}, \mathrm{CH}_{\text {bridge- }}\right.$ head $), 46.0\left(\mathrm{~d}, J_{\mathrm{CP}}=4.0 \mathrm{~Hz}, C \mathrm{H}_{2}\right), 28.7\left(\mathrm{~s}, C \mathrm{H}_{\mathrm{iPr}}\right), 28.5\left(\mathrm{~s}, C \mathrm{H}_{\mathrm{PPr}}\right), 28.3$ $\left(\mathrm{s}, C \mathrm{H}_{2}\right), 26.4\left(\mathrm{~d},{ }^{3} J_{\mathrm{CP}}=2.5 \mathrm{~Hz}, C \mathrm{H}_{2}\right), 25.4\left(\mathrm{~s}, C \mathrm{H}_{3 \mathrm{PPr}}\right), 25.0\left(\mathrm{~s}, C \mathrm{H}_{3 \mathrm{PPr}}\right)$, $24.7\left(\mathrm{~s}, \mathrm{CH}_{3 \mathrm{iPr}}\right), 24.0\left(\mathrm{~s}, C_{3 \mathrm{iPr}}\right),-1.7\left(\mathrm{~s}, \mathrm{Si}-\mathrm{CH}_{3}\right),-3.5\left(\mathrm{~s}, \mathrm{Si}-\mathrm{CH}_{3}\right) .{ }^{31} \mathrm{P}$ NMR (202 MHz, $\mathrm{CD}_{2} \mathrm{Cl}_{2}$ ): $\left.\delta=22.2(\mathrm{~s}) .{ }^{19} \mathrm{~F} \mathrm{NMR} \mathrm{(471} \mathrm{MHz,} \mathrm{CD}_{2} \mathrm{Cl}_{2}\right)$ : $\delta=-133.0\left(\mathrm{br}, \mathrm{C}_{\mathrm{Ar}}-F_{\text {ortho }}\right),-163.7\left(\mathrm{t}, J_{\mathrm{FF}}=20.4 \mathrm{~Hz}, \mathrm{C}_{\mathrm{Ar}}-F_{\text {para }}\right),-167.5(\mathrm{t}$, $\left.J_{\mathrm{FF}}=18.1 \mathrm{~Hz}, \mathrm{C}_{\mathrm{Ar}}-F_{\text {meta }}\right) .{ }^{11} \mathrm{~B} \mathrm{NMR}\left(160 \mathrm{MHz}, \mathrm{CD}_{2} \mathrm{Cl}_{2}\right): \delta=-16.6(\mathrm{~s}$, $B$ Ar). ${ }^{29} \mathrm{Si} \mathrm{NMR}\left(99 \mathrm{MHz}, \mathrm{CD}_{2} \mathrm{Cl}_{2}\right): \delta=8.9$ (s).

\section{ASSOCIATED CONTENT}

\section{Supporting Information}

The Supporting Information is available free of charge on the ACS Publications website.

Spectroscopic and X-ray data (PDF), X-ray of: 3 (CIF); $4 \mathbf{b}$ (CIF); 4c (CIF); 4d (CIF), 4e (CIF) and 7 (CIF).

\section{AUTHOR INFORMATION}

\section{Corresponding Authors}

Tsuyoshi Kato - Université de Toulouse, UPS, and CNRS, LHFA UMR 5069, 118 route de Narbonne, 31062 Toulouse, France ; Email : kato@chimie.ups-tlse.fr

Eddy Maerten - Université de Toulouse, UPS, and CNRS, LHFA UMR 5069, 118 route de Narbonne, 31062 Toulouse, France ; Email : maerten@ chimie.ups-tlse.fr

\section{Authors}


Aymeric Dajnak - Université de Toulouse, UPS, and CNRS, LHFA UMR 5069, 118 route de Narbonne, 31062 Toulouse,

France

Antoine Baceiredo - Université de Toulouse, UPS, and CNRS, LHFA UMR 5069, 118 route de Narbonne, 31062 Toulouse, France

Nathalie Saffon-Merceron - Université de Toulouse, UPS, and CNRS, ICT FR2599 118 route de Narbonne, 31062 Toulouse,

France

Complete contact information is available at: https://pubs.acs.org/

Author Contributions A. D. performed all synthetic work, while N. S. M. performed all X-ray structural studies.

\section{Notes}

The authors declare no competing financial interest.

\section{ACKNOWLEDGMENT}

The Agence Nationale de la Recherche (ANR-16-CE07-0018-01) and the Ministère de l'Enseignement Supérieur et de la Recherche are gratefully acknowledged for Ph.D. grant to A. D. The authors would like to thanks the CNRS and the Université de Toulouse, UPS for financial support.

\section{REFERENCES}

(1) Kim, K.-C.; Reed, C. A.; Elliott, D. W.; Mueller, L. J.; Tham, F.; Lin, L.; Lambert, J. B. Crystallographic Evidence for a Free Silylium Ion. Science 2002, 297, 825-827.

(2) a) Müller, T. A model system for the generation of silyl cationic species of different reactivity and stability. In Organosilicon Chemistry V, N. Auner, J. Weis, Eds.; Wiley-VCH: Weinheim (2003); p. 34-44. b) Klare, H. F. T.; Oestreich, M. Silylium ions in catalysis. Dalton Trans. 2010, 39, 9176-9184. c) Sekiguchi, A.; Lee, V. Y. Organometallic compounds of low-coordinate $\mathrm{Si}, \mathrm{Ge}, \mathrm{Sn}$ and $\mathrm{Pb}, \mathbf{2 0 1 0}$, Wiley, Chichester. d) Müller, T. Silylium ions and stabilized silylium ions in Science of Synthesis, Knowledge updates 2013/3, Vol. ed. Oestreich, M.; Thieme, G. Verlag KG, Stuttgart, 2013, 1-42. e) Müller, T. Silylium ions in Struct. Bond 155, Vol. Ed. Scheschkewitz, D. 2014, 107-162.

(3) a) Siegel, J. S. Silylium ions: from controversial beginnings to useful catalysts. Nature Reviews Chemistry 2020, 4, 4-5. b) Walker, J. C. L.; Klare, H. F. T.; Oestreich, M. Cationic silicon Lewis acids in catalysis. Nature Reviews Chemistry 2020, 4, $54-62$.

(4) a) Hara, K.; Akiyama, R.; Sawamura, M. Strong Counteranion Effects on the Catalytic Activity of Cationic Silicon Lewis Acids in Mukaiyama Aldol and Diels-Alder Reactions. Org. Lett. 2005, 7, 5621-5623. b) Duttwyler, S.; Do, Q.-Q.; Linden, A.; Baldridge, K. K.; Siegel, J. S. Synthesis of 2,6-diarylphenyldimethylsilyl cations: polar-pi distribution of cation character. Angew. Chem. Int. Ed, 2008, 47, 1719-1722. c) Romanato, P.; Duttwyler, S.; Linden, A.; Baldridge, K. K.; Siegel, J. S. Intramolecular Halogen Stabilization of Silylium Ions Directs Gearing Dynamics. J. Am. Chem. Soc. 2010, 132, 7828-7829. d) Romanato, P.; Duttwyler, S.; Linden, A.; Baldridge, K. K.; Siegel, J. S. Competition between $\pi$-Arene and Lone-Pair Halogen Coordination of Silylium Ions? J. Am. Chem. Soc. 2011, 133, 11844-11846. e) Schäfer, A.; Saak, W.; Haase, D.; Müller, T. Silyl cation mediated conversion of CO2 into benzoic acid, formic acid, and methanol. Angew. Chem. Int. Ed. Engl., 2012, 51, 2981-2984. f) Ducos, P.; Liautard, V.; Robert, F.; Landais, Y. Chiral Memory in Silylium Ions. Chem. A Eur. J. 2015, 21, 11573-11578. g) Fernandes, A.; Laye, C.; Pramanik, S.; Palmeira, D.; Pekel, O. P.; Massip, S.; Schmidtmann, M.; Müller, T.; Robert, F.; Landais, Y. Chiral Memory in Silyl-Pyridinium and Quinolinium Cations. J. Am.
Chem. Soc. 2020, 142, 564-572. h) Wu, Q.; Roy, A.; Wang, G.; Irran, E.; Klare, H. F. T.; Oestreich, M. Synthesis of a Counteranion-Stabilized Bis(silylium) Ion. Angew. Chem. Int. Ed. 2020, 59, 10523-10526. i) Künzler, S.; Rathjen, S.; Rüger, K.; Würdemann, M. S.; Wernke, M.; Tholen, P.; Girschik, C.; Schmidtmann, M.; Landais, Y.; Müller, T. Chiral ChalcogenylSubstituted Naphthyl- and Acenaphthyl-silanes and their Cations. Chem. Eur. J doi: 10.1002/chem.202002977.

(5) a) Scott, V. J.; Remle, Ç.-Ç.; Ozerov, O. V. Room-Temperature Catalytic Hydrodefluorination of $\mathrm{C}\left(\mathrm{sp}^{3}\right)-\mathrm{F}$ Bonds. J. Am. Chem. Soc. 2005, 127, 2852-2853. b) Panisch, R.; Bolte, M.; Müller, T. Hydrogen- and Fluorine-Bridged Disilyl Cations and Their Use in Catalytic C-F Activation. J. Am. Chem. Soc. 2006, 128, 9676-9682. c) Douvris, C.; Ozerov, O. V. Hydrodefluorination of Perfluoroalkyl Groups Using Silylium-Carborane Catalysts. Science, 2008, 321, 1188-1190. d) Douvris, C.; Nagaraja, C. M.; Chen, C.-H.; Foxman, B. M.; Ozerov, O. V. Hydrodefluorination and Other Hydrodehalogenation of Aliphatic Carbon-Halogen Bonds Using Silylium Catalysis. $J$. Am. Chem. Soc. 2010, 132, 4946-4953. e) Lühmann, N.; Panisch, R.; Müller, T. A catalytic C-C bond-forming reaction between aliphatic fluorohydrocarbons and arylsilanes. Appl. Organomet. Chem. 2010, 24, 533-537. f) Lühmann, N.; Hirao, H.; Shaik, S.; Müller, T. Disilylfluoronium Ions-Synthesis, Structure, and Bonding. Organometallics 2011, 30, 4087-4096. g) Allemann, O.; Duttwyler, S.; Baldridge, K. K.; Siegel, J. S. Proton-Catalyzed, Silane-Fueled Friedel-Crafts Coupling of Fluoroarenes. Science 2011, 332, 574-577. h) Stahl, T.; Klare, H. F. T.; Oestreich, M. Main-Group Lewis Acids for C-F Bond Activation. ACS Catal. 2013, 3, 1578-1587. i) Kordts, N.; Borner, C.; Panisch, R.; Saak, W.; Müller, T. Hydrogen-Bridged Digermyl and Germylsilyl Cations. Organometallics 2014, 33, 1492-1498. j) Kordts, N.; Künzler, S.; Rathjen, S.; Sieling, T.; Großekappenberg, H.; Schmidtmann, M.; Müller, T. Silyl Chalconium Ions: Synthesis, Structure and Application in Hydrodefluorination Reactions. Chem. Eur. J. 2017, 23, 10068-10079.

(6) a) Klare, H. F. T.; Bergander, K.; Oestreich, M. Taming the Silylium Ion for Low-Temperature Diels-Alder Reactions. An gew. Chem. Int. Ed. 2009, 48, 9077-9079. b) Müther, K.; Fröhlich, R.; Mück-Lichtenfeld, C.; Grimme, S.; Oestreich, M. A Unique Transition Metal-Stabilized Silicon Cation. J. Am. Chem. Soc. 2011, 133, 12442-12444. c) Müther, K.; Oestreich, M. Self-regeneration of a silylium ion catalyst in carbonyl reduction. Chem. Commun. 2011, 47, 334-336. d) Schmidt, R. K.; Müther, K.; Mück-Lichtenfeld, C.; Grimme, S.; Oestreich, M. Silylium Ion-Catalyzed Challenging Diels-Alder Reactions: The Danger of Hidden Proton Catalysis with Strong Lewis Acids. J. Am. Chem. Soc. 2012, 134, 4421-4428. e) Müther, K.; Hrobárik, P.; Hrobáriková, V.; Kaupp, M.; Oestreich, M. The Family of Ferrocene-Stabilized Silylium Ions: Synthesis, 29Si NMR Characterization, Lewis Acidity, Substituent Scrambling, and Quantum-Chemical Analyses. Chem. Eur. J. 2013, 19, 16579-16594. f) Müther, K.; Mohr, J.; Oestreich, M. Silylium Ion Promoted Reduction of Imines with Hydrosilanes. Organometallics 2013, 32, 6643-6646.

(7) a) Schäfer, A.; Reißmann, M.; Schäfer, A.; Saak, W.; Haase, D.; Müller, T. A New Synthesis of Triarylsilylium Ions and Their Application in Dihydrogen Activation. Angew. Chem. Int. Ed. 2011, 50, 12636-12638. b) Reißmann, M.; Schäfer, A.; Jung, S.; Müller, T. Silylium Ion/Phosphane Lewis Pairs. Organometallics 2013, 32, 6736-6744

(8) a) Igau, A.; Grutzmacher, H.; Baceiredo, A.; Bertrand, G Analogous alpha.,.alpha.'-bis-carbenoid, triply bonded species: synthesis of a stable .lambda.3-phosphino carbene-.lambda.5phosphaacetylene. J. Am. Chem. Soc. 1988, 110, 6463-6466. b) Lavigne, F.; Maerten, E.; Alcaraz, G.; Saffon-Merceron, N.; Acosta-Silva, C.; Branchadell, V.; Baceiredo, A. Borylated Methylenephosphonium Salts: Precursors of Elusive Boryl(phosphino)carbenes. J. Am. Chem. Soc. 2010, 132, 8864-8865. c) Lavigne, F.; El Kazzi, A.; Escudié, Y.; Maerten, 
E.; Kato, T.; Saffon-Merceron, N.; Branchadell, V.; Cossio, F. P.; Baceiredo, A. Azavinylidenephosphoranes: a class of cyclic push-pull carbenes. Chem. Eur. J. 2014, 20, 12528-12536.

(9) Gau, D.; Kato, T.; Saffon-Merceron, N.; Cossío, F. P.; Baceiredo, A. Stable Phosphonium Sila-ylide with Reactivity as a Sila-Wittig Reagent. J. Am. Chem. Soc. 2009, 131, 8762-8763.

(10) a) Rodriguez, R.; Gau, D.; Contie, Y.; Kato, T.; Saffon-Merceron, N.; Baceiredo, A. Synthesis of a Phosphine-Stabilized Silicon(II) Hydride and Its Addition to Olefins: A CatalystFree Hydrosilylation Reaction. Angew. Chem. Int. Ed. 2011, 50, 11492-11495. b) Rodriguez, R.; Gau, D.; Kato, T.; SaffonMerceron, N.; De Cozar, A.; Cossio, F. P.; Baceiredo, A. Reversible binding of ethylene to silylene-phosphine complexes at room temperature. Angew. Chem. Int. Ed. 2011, 50, 10414-10416.

(11) a) Rodriguez, R.; Contie, Y.; Nougué, R.; Baceiredo, A.; Saffon-Merceron, N.; Sotiropoulos, J.-M.; Kato, T. Reversible Silylene Insertion Reactions into $\mathrm{Si}-\mathrm{H}$ and $\mathrm{P}-\mathrm{H} \sigma$-Bonds at Room Temperature. Angew.Chem.Int. Ed. 2016, 55, 14355-14358. b) Nakagaki, M.; Baceiredo, A.; Kato, T.; Sakaki, S. Reversible Oxidative Addition/Reductive Elimination of a $\mathrm{Si}-\mathrm{H}$ Bond with Base-Stabilized Silylenes: A Theoretical Insight. Chem. Eur. J. 2018, 24, 11377-11385.

(12) See a) Cabrera, L.; Welch, G. C.; Masuda, J. D.; Wei, P.; Stephan, D. W. Pyridine and phosphine reactions with $\left[\mathrm{CPh}_{3}\right]\left[\mathrm{B}\left(\mathrm{C}_{6} \mathrm{~F}_{5}\right)_{4}\right]$. Inorg. Chim. Acta 2006, 359, 3066-3071 and earliest research: b) Hoffmann, H.; Schellenbeck, P. Darstellung und Eigenschaften einiger Phosphorverbindungen mit tert.-Butylgruppen. Chem. Ber. 1966, 99, 1134-1142. c) Sanders, J. R. Preparation of hydridotetrakis(triphenylphosphine)ruthenium(II) hexafluorophosphate and related complexes. $J$. Chem. Soc., Dalton Trans 1973, 743-749. d) Lambert, J. B.; So, J. H. Phosphonium ions rather than phosphenium ions from the reaction of secondary phosphines with trityl cation. J. Org. Chem. 1991, 56, 5960-5962. e) Fang, X.; Scott, B. L.; John, K. D.; Kubas, G. J.; Watkin, J. G. Triphenylmethyl tris(p-methoxyphenyl)phosphonium cation: a structurally characterized phosphonium analog of hexaphenylethane. New J. Chem. 2000, 24, 831-833.

(13) See a) Kira, M.; Hino, T.; Sakurai, H. Chemistry of organosilicon compounds. 292. An NMR study of the formation of silyloxonium ions by using tetrakis[3,5-bis(trifluoromethyl)phenyl]borate as counteranion. J. Am. Chem. Soc. 1992, 114, 6697-6700 and also b) Ishida, S.; Nishinaga, T.; West, R.; Komatsu, K. Generation and aromaticity of 2-silaimidazolium ion, a new $\pi$-conjugated silylium ion. Chem. Commun. 2005, $778-780$
(14) Olah, G. A.; Field, L. D. Organometallic chemistry. 19. Correlation of silicon-29 and carbon-13 NMR chemical shifts in analogous compounds. Application in attempted preparation of silicenium ions. Organometallics 1982, 1, 1485-1487.

(15) a) Johansen, M.; Jorgensen, K. A.; Helmchen, G. Synthesis and Application of the First Chiral and Highly Lewis Acidic Silyl Cationic Catalyst. J. Am. Chem. Soc. 1998, 120, 7637-7638. b) Shaykhutdinova, P.; Oestreich, M. Enantioselective Diels-Alder Reactions of Cyclohexa-1,3-diene and Chalcones Catalyzed by Intramolecular Silicon-Sulfur Lewis Pairs as Chiral Lewis Acids. Organometallics 2016, 35, 2768-2771.

(16) a) Stephan, D. W.; Erker, G. Frustrated Lewis Pairs: Metal-free Hydrogen Activation and More. Angew. Chem. Int. Ed. 2010 , 49, 46-76. b) Erker, G. Frustrated Lewis pairs: Reactions with dihydrogen and other "small molecules". C. R. Chim. 2011, 14, 831-841. c) Stephan, D. W.; Greenberg S.; Graham, T. W.; Chase, P.; Hastie, J.-J.; Geier, S. J.; Farrell, J. M. Brown, C. C.; Heiden, Z. M.; Welch, G. C.; Ullrich, M. Metal-Free Catalytic Hydrogenation of Polar Substrates by Frustrated Lewis Pairs. Inorg. Chem. 2011, 50, 12338-12348. d) Erker, G. Organometallic frustrated Lewis pair chemistry. Dalton Trans. 2011, 40, 7475-7483. e) Stephen, D. W. "Frustrated Lewis pair" hydrogenations. Org. Biomol. Chem. 2012, 10, 5740-5746.

(17) Silylium ions 4 do not react with benzaldehyde.

(18) Hupf, E.; Olaru, M.; Rat, C. I.; Fugel, M. Hübschle, C. B.; Lork, E; Grabowsky, S; Mebs, S.; Beckmann, J. Mapping the Trajectory of Nucleophilic Substitution at Silicon Using a periSubstituted Acenaphthyl Scaffold. Chem. Eur. J. 2017, 23, 10568-10579.

(19) Schneider, J.; Sindlinger, C. P.; Freitag, S. M.; Schubert, H.; Wesemann, L. Diverse Activation Modes in the Hydroboration of Aldehydes and Ketones with Germanium, Tin, and Lead Lewis Pairs. Angew. Chem. Int. Ed. 2017, 56, 333-337.

(20) Baceiredo, A.; Kato, T.; Mao, Y.; Berthe, J.; Bousquié, M. Method of hydrosilylation implementing an organic catalyst derived from germylene. PATENT US 2017/0313729A1, 2017/11/02, 2017.

(21) Guan, Z.; Marshall, W. J. Synthesis of New Phosphine Imine Ligands and Their Effects on the Thermal Stability of LateTransition-Metal Olefin Polymerization Catalysts. Organometallics 2002, 21, 3580-3586.

(22) Gau, D.; Kato, T.; Saffon-Merceron, N.; De Cezar, A.; Cossio, F. P.; Baceiredo, A. Synthesis and Structure of a Base-Stabilized C-Phosphino-Si-Amino Silyne. Angew. Chem. Int. Ed. 2010, 49, 6585-6588.

\section{Table of Contents artwork}

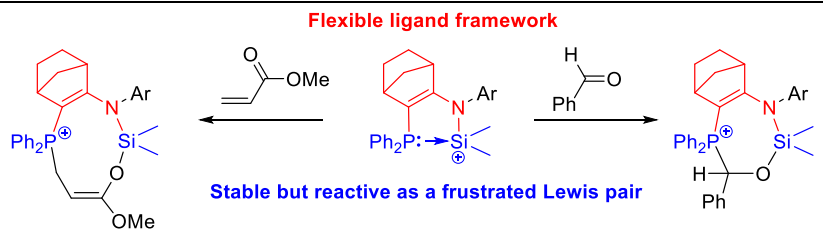

